\title{
MORTAR FINITE ELEMENT DISCRETIZATION OF A MODEL COUPLING DARCY AND STOKES EQUATIONS
}

\author{
Christine Bernardi ${ }^{1}$, Tomás Chacón Rebollo ${ }^{1,2}$, Frédéric Hecht ${ }^{1}$ \\ AND Zoubida MGHAZLi ${ }^{3}$
}

\begin{abstract}
As a first draft of a model for a river flowing on a homogeneous porous ground, we consider a system where the Darcy and Stokes equations are coupled via appropriate matching conditions on the interface. We propose a discretization of this problem which combines the mortar method with standard finite elements, in order to handle separately the flow inside and outside the porous medium. We prove a priori and a posteriori error estimates for the resulting discrete problem. Some numerical experiments confirm the interest of the discretization.
\end{abstract}

Mathematics Subject Classification. 65N30, 65N55, 76M10.

Received January 8, 2007. Revised October 3, 2007.

Published online April 1st, 2008.

\section{INTRODUCTION}

We first describe the model we intend to work with. Let $\Omega$ be a rectangle in dimension $d=2$ or a rectangular parallelepiped in dimension $d=3$. We assume that it is divided (without overlap) into two connected open sets $\Omega_{P}$ and $\Omega_{F}$ with Lipschitz-continuous boundaries, where the indices $P$ and $F$ stand for porous and fluid, respectively. The fluid that we consider is viscous and incompressible. So in the porous medium, which is assumed to be rigid and saturated with the fluid, we consider the following equations, due to Darcy,

$$
\begin{cases}\alpha \boldsymbol{u}+\operatorname{grad} p=\boldsymbol{f} & \text { in } \Omega_{P} \\ \operatorname{div} \boldsymbol{u}=0 & \text { in } \Omega_{P}\end{cases}
$$

In $\Omega_{F}$, the flow of this same fluid is governed by the Stokes equations

$$
\begin{cases}-\nu \Delta \boldsymbol{u}+\operatorname{grad} p=f & \text { in } \Omega_{F} \\ \operatorname{div} \boldsymbol{u}=0 & \text { in } \Omega_{F}\end{cases}
$$

\footnotetext{
Keywords and phrases. Mortar method, finite elements, Darcy equations, Stokes equations.

1 Laboratoire Jacques-Louis Lions, C.N.R.S. \& Université Pierre et Marie Curie, B.C. 187, 4 place Jussieu, 75252 Paris Cedex 05, France. bernardi@ann.jussieu.fr; hecht@ann.jussieu.fr

2 Departamento de Ecuaciones Diferenciales y Análisis Numerico, Universidad de Sevilla, Tarfia s/n, 41012 Sevilla, Spain. chacon@numer.us.es

3 Équipe d'Ingénierie Mathématique, LIRNE, Faculté des Sciences, Université Ibn Tofail, B.P. 133, Kénitra, Morocco. mghazli_zoubida@yahoo.com
} 


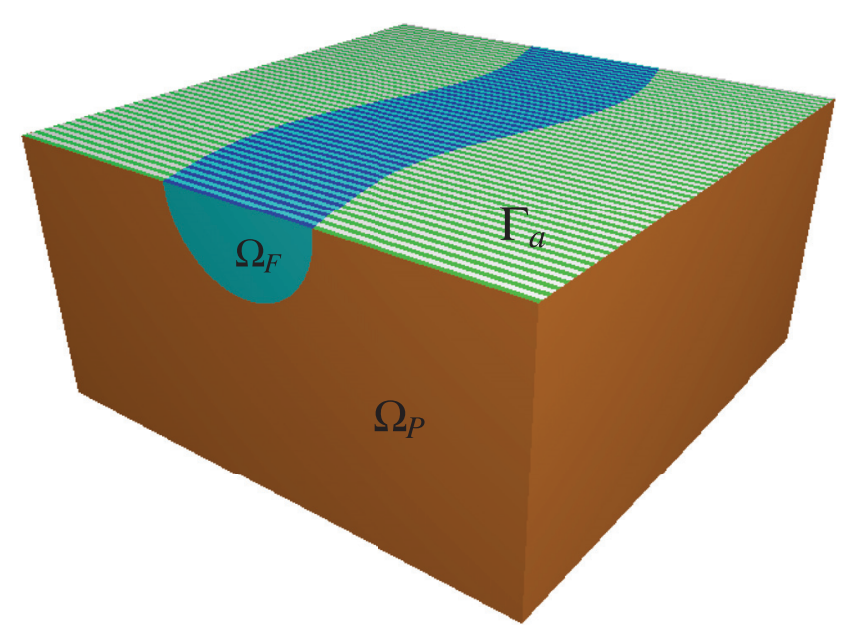

Figure 1. An example of three-dimensional domain $\Omega$.

The unknowns both in (1.1) and (1.2) are the velocity $\boldsymbol{u}$ and the pressure $p$ of the fluid. The parameters $\nu$ and $\alpha$ are positive constants, representing the viscosity of the fluid and the ratio of this viscosity to the permeability of the medium, respectively. The porous medium is supposed to be homogeneous, so that we take $\alpha$ constant on the whole subdomain $\Omega_{P}$ (we refer to [1] and [12] for handling the somewhat more realistic case where $\alpha$ is piecewise constant in a different framework). Note also that the deformation tensor is replaced by the gradient operator in (1.2) for mathematical simplicity (this simplification is standard in geophysics, see e.g. [29], Sect. 1.2.3).

Concerning the boundary conditions, as illustrated in Figure $1(d=3)$ and also in Figure $2(d=2)$ with more details, we denote by $\Gamma_{a}$ the upper edge $(d=2)$ or face $(d=3)$ of $\Omega$, where the index a means in contact with the atmosphere. Let $\Gamma_{a P}$ be the intersection $\Gamma_{a} \cap \partial \Omega_{P}$ and $\Gamma_{a F}$ the intersection $\Gamma_{a} \cap \partial \Omega_{F}$ (note that $\Gamma_{a P}$ can be empty in some practical situations). We set:

$$
\Gamma_{P}=\left(\partial \Omega \cap \partial \Omega_{P}\right) \backslash \Gamma_{a P} \quad \text { and } \quad \Gamma_{F}=\left(\partial \Omega \cap \partial \Omega_{F}\right) \backslash \Gamma_{a F} .
$$

Let $\boldsymbol{n}$ stand for the unit outward normal vector to $\Omega$ on $\partial \Omega$ and also to $\Omega_{P}$ on $\partial \Omega_{P}$. We provide the previous partial differential equations (1.1) and (1.2) with the conditions

$$
\boldsymbol{u} \cdot \boldsymbol{n}=k \quad \text { on } \Gamma_{P} \quad \text { and } \quad p=p_{a} \quad \text { on } \Gamma_{a P},
$$

and

$$
\boldsymbol{u}=\boldsymbol{g} \quad \text { on } \Gamma_{F} \quad \text { and } \quad \nu \partial_{n} \boldsymbol{u}-p \boldsymbol{n}=\boldsymbol{t}_{a} \quad \text { on } \Gamma_{a F} .
$$

Note that these conditions are of Dirichlet type on $\partial \Omega \backslash \Gamma_{a}$, while the condition on $\Gamma_{a P}$ only means that the pressure, here equal to $p_{a}$, depends on the atmospheric pressure. The condition on $\Gamma_{a F}$ means that the variations of the free surface at the top of the flow are neglected in the model. Thus $\boldsymbol{t}_{a}$ mainly depends on the atmospheric pressure and the wind on the river. This is standard in geophysics, see e.g. [29], Section 1.4; note however that, when the flux $\int_{\Gamma_{F}}(\boldsymbol{g} \cdot \boldsymbol{n})(\boldsymbol{\tau}) \mathrm{d} \boldsymbol{\tau}+\int_{\Gamma_{P}} k(\boldsymbol{\tau}) \mathrm{d} \boldsymbol{\tau}$ is too large, this boundary condition is not compatible with the physics of the problem.

To conclude, let $\Gamma$ denote the interface $\partial \Omega_{P} \cap \partial \Omega_{F}$. On $\Gamma$ we consider the matching conditions

$$
\left.\boldsymbol{u}\right|_{\Omega_{P}} \cdot \boldsymbol{n}=\left.\boldsymbol{u}\right|_{\Omega_{F}} \cdot \boldsymbol{n} \quad \text { and } \quad-\left.p\right|_{\Omega_{P}} \boldsymbol{n}=\left.\nu \partial_{n} \boldsymbol{u}\right|_{\Omega_{F}}-\left.p\right|_{\Omega_{F}} \boldsymbol{n} \quad \text { on } \Gamma .
$$



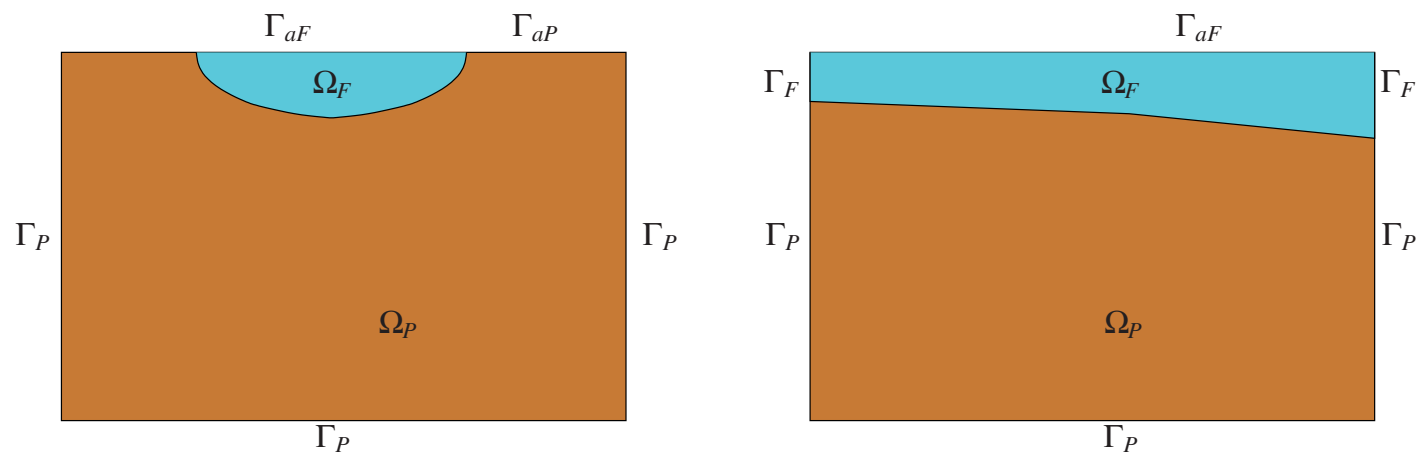

Figure 2. Two examples of two-dimensional domains $\Omega$.

Indeed, from a physical point of view, conservation of mass enforces continuity of the normal velocities at the interface. Similarly, conservation of momentum enforces continuity of the normal stresses. Such interface conditions are studied for instance in [21,28] and [17], Section 4.5. Note that a lot of recent works deal with the coupling of Darcy and Stokes equations in different frameworks, hence with other types of matching conditions such as the Beaver-Joseph-Saffman conditions, see [10,16,21,23,28,35] and the references therein.

System (1.1)-(1.5) is only a first draft of a model for the laminar flow of a river over a porous rock such as limestone, however it seems that its discretization has not been considered before. Of course, in more realistic models, the Stokes equations must be replaced by the Navier-Stokes equations (for instance when the river meets obstacles) and the Darcy equations must be replaced by more complex models as proposed in [33] (see also [4] or [20]). However we are interested with this system. We first write an equivalent variational formulation of it and prove that it admits a unique solution.

The discretization that we propose relies on the mortar element method, a domain decomposition technique introduced in [7] (see also [11] for the new trends). Indeed it seems convenient to use a subdomain for the fluid and another one for the porous medium. Moreover, owing to the flexibility of the mortar method, independent meshes can be used on the different parts of the domain. On each subdomain, we consider a finite element discretization, relying on standard finite elements both for the Stokes problem (the element first introduced in [22] and analyzed in [6]) and the Darcy equations (the Raviart-Thomas element [34]). These choices can be justified as follows:

- The Raviart-Thomas element is the simplest and less expensive element which is conforming in the domain of the divergence operator, so that we use it on $\Omega_{P}$.

- It is usually associated with piecewise constant pressures, in order that the inf-sup condition linking the two spaces on $\Omega_{P}$ is optimal (where "optimal" means "with a constant independent of the discretization parameter").

- Thus, for simplicity, piecewise constant pressures are used on the whole domain.

- The Bernardi-Raugel element is the less expensive element which, when associated with the space of piecewise constant pressures, leads to an optimal inf-sup condition on $\Omega_{F}$.

We construct a discrete problem and we check that it has a unique solution. We then prove optimal a priori and a posteriori upper bounds for the error, despite the lack of conformity of the mortar method.

Thanks to the error indicators issued from the a posteriori analysis, we are in a position to perform mesh adaptivity independently in the porous and fluid domain. We describe the adaptivity strategy that we use. Next we present numerical experiments. The results are in good agreement with the error estimates, so they justify our choice of discretization. 
The outline of the paper is as follows.

- In Section 2, we write the variational formulation of the problem and prove its well-posedness.

- Section 3 is devoted to the description of the discrete problem and to the proof of its well-posedness.

- We prove the a priori and a posteriori estimates in Sections 4 and 5 , respectively.

- The adaptivity strategy and numerical experiments are presented in Section 6.

\section{AnAlysis of the MODEL}

We first intend to write a variational formulation of system (1.1)-(1.5). From now on, for each domain $\mathcal{O}$ in $\mathbb{R}^{d}$ with a Lipschitz-continuous boundary, we use the full scale of Sobolev spaces $H^{s}(\mathcal{O})$ and $H_{0}^{s}(\mathcal{O}), s \geq 0$, their trace spaces on $\partial \mathcal{O}$ and their dual spaces. We denote by $\mathscr{C}^{\infty}(\overline{\mathcal{O}})$ the space of restrictions to $\mathcal{O}$ of indefinitely differentiable functions on $\mathbb{R}^{d}$ and by $\mathscr{D}(\mathcal{O})$ its subspace made of functions with a compact support in $\mathcal{O}$.

Let also $H(\operatorname{div}, \Omega)$ denote the space of functions $\boldsymbol{v}$ in $L^{2}(\Omega)^{d}$ such that $\operatorname{div} \boldsymbol{v}$ belongs to $L^{2}(\Omega)$, equipped with the norm

$$
\|\boldsymbol{v}\|_{H(\operatorname{div}, \Omega)}=\left(\|\boldsymbol{v}\|_{L^{2}(\Omega)^{d}}^{2}+\|\operatorname{div} \boldsymbol{v}\|_{L^{2}(\Omega)}^{2}\right)^{\frac{1}{2}} .
$$

We recall the Stokes formula, valid for smooth enough functions $\boldsymbol{v}$ and $q$,

$$
\int_{\Omega}(\operatorname{div} \boldsymbol{v})(\boldsymbol{x}) q(\boldsymbol{x}) \mathrm{d} \boldsymbol{x}+\int_{\Omega} \boldsymbol{v}(\boldsymbol{x}) \cdot(\operatorname{grad} q)(\boldsymbol{x}) \mathrm{d} \boldsymbol{x}=\int_{\partial \Omega}(\boldsymbol{v} \cdot \boldsymbol{n})(\boldsymbol{\tau}) q(\boldsymbol{\tau}) \mathrm{d} \boldsymbol{\tau} .
$$

Since $\mathscr{C}^{\infty}(\bar{\Omega})^{d}$ is dense in $H(\operatorname{div}, \Omega)$ [24], Chapter I, Theorem 2.4, we derive from this formula that the normal trace operator: $\boldsymbol{v} \mapsto \boldsymbol{v} \cdot \boldsymbol{n}$ is defined and continuous from $H(\operatorname{div}, \Omega)$ into $H^{-\frac{1}{2}}(\partial \Omega)$. This leads to define

$$
H_{0}(\operatorname{div}, \Omega)=\{\boldsymbol{v} \in H(\operatorname{div}, \Omega) ; \boldsymbol{v} \cdot \boldsymbol{n}=0 \text { on } \partial \Omega\}
$$

Then $\mathscr{D}(\Omega)^{d}$ is dense in $H_{0}(\operatorname{div}, \Omega)$ [24], Chapter I, Theorem 2.6, and both $H(\operatorname{div}, \Omega)$ and $H_{0}(\operatorname{div}, \Omega)$ are Hilbert spaces for the scalar product associated with the norm defined in (2.1).

Remark 2.1. Let $\Gamma^{*}$ be any part of $\partial \Omega$ with positive measure. We refer to [30], Chapter 1 , Section 11 , for the definition of $H_{00}^{\frac{1}{2}}\left(\Gamma^{*}\right)$ as the space of functions in $H^{\frac{1}{2}}\left(\Gamma^{*}\right)$ such that their extension by zero belongs to $H^{\frac{1}{2}}(\partial \Omega)$. The normal trace on $\Gamma^{*}$ of a function $\boldsymbol{v}$ in $H(\operatorname{div}, \Omega)$ makes sense in $H_{00}^{\frac{1}{2}}\left(\Gamma^{*}\right)^{\prime}$, owing to the following formula

$$
\forall q \in H_{00}^{\frac{1}{2}}\left(\Gamma^{*}\right), \quad \int_{\Gamma^{*}}(\boldsymbol{v} \cdot \boldsymbol{n})(\boldsymbol{\tau}) q(\boldsymbol{\tau}) \mathrm{d} \boldsymbol{\tau}=\int_{\Omega}(\operatorname{div} \boldsymbol{v})(\boldsymbol{x}) \bar{q}(\boldsymbol{x}) \mathrm{d} \boldsymbol{x}+\int_{\Omega} \boldsymbol{v}(\boldsymbol{x}) \cdot(\operatorname{grad} \bar{q})(\boldsymbol{x}) \mathrm{d} \boldsymbol{x},
$$

where $\bar{q}$ is any lifting in $H^{1}(\Omega)$ of the extension by zero of $q$ to $\partial \Omega$ (clearly the integral in the left-hand side of the previous equality represents a duality pairing). Note moreover that $H^{-\frac{1}{2}}\left(\Gamma^{*}\right)$ is imbedded in $H_{00}^{\frac{1}{2}}\left(\Gamma^{*}\right)^{\prime}$.

We now introduce the variational spaces

$$
\begin{aligned}
X(\Omega) & =\left\{\boldsymbol{v} \in H(\operatorname{div}, \Omega) ; \boldsymbol{v}_{\mid \Omega_{F}} \in H^{1}\left(\Omega_{F}\right)^{d}\right\}, \\
X_{0}(\Omega) & =\left\{\boldsymbol{v} \in X(\Omega) ; \boldsymbol{v} \cdot \boldsymbol{n}=0 \text { on } \Gamma_{P} \text { and } \boldsymbol{v}=\mathbf{0} \text { on } \Gamma_{F}\right\} .
\end{aligned}
$$

Both of them are equipped with the norm

$$
\|\boldsymbol{v}\|_{X(\Omega)}=\left(\|\boldsymbol{v}\|_{H\left(\operatorname{div}, \Omega_{P}\right)}^{2}+\|\boldsymbol{v}\|_{H^{1}\left(\Omega_{F}\right)}^{2}\right)^{\frac{1}{2}}
$$


and are Hilbert spaces for the corresponding scalar product. We also consider the bilinear forms

$$
\begin{gathered}
a(\boldsymbol{u}, \boldsymbol{v})=a_{P}(\boldsymbol{u}, \boldsymbol{v})+a_{F}(\boldsymbol{u}, \boldsymbol{v}) \\
\text { with } \quad a_{P}(\boldsymbol{u}, \boldsymbol{v})=\alpha \int_{\Omega_{P}} \boldsymbol{u}(\boldsymbol{x}) \cdot \boldsymbol{v}(\boldsymbol{x}) \mathrm{d} \boldsymbol{x}, \\
a_{F}(\boldsymbol{u}, \boldsymbol{v})=\nu \int_{\Omega_{F}}(\operatorname{grad} \boldsymbol{u})(\boldsymbol{x}):(\operatorname{grad} \boldsymbol{v})(\boldsymbol{x}) \mathrm{d} \boldsymbol{x}, \\
b(\boldsymbol{v}, q)=-\int_{\Omega}(\operatorname{div} \boldsymbol{v})(\boldsymbol{x}) q(\boldsymbol{x}) \mathrm{d} \boldsymbol{x} .
\end{gathered}
$$

It is readily checked that the first three forms are continuous on $X(\Omega) \times X(\Omega)$, while the last one is continuous on $X(\Omega) \times L^{2}(\Omega)$.

The variational problem that we consider now reads:

Find $(\boldsymbol{u}, p)$ in $X(\Omega) \times L^{2}(\Omega)$ such that

$$
\boldsymbol{u} \cdot \boldsymbol{n}=k \quad \text { on } \Gamma_{P} \quad \text { and } \quad \boldsymbol{u}=\boldsymbol{g} \quad \text { on } \Gamma_{F},
$$

and that

$$
\begin{aligned}
& \forall \boldsymbol{v} \in X_{0}(\Omega), \quad a(\boldsymbol{u}, \boldsymbol{v})+b(\boldsymbol{v}, p)=\mathcal{L}(\boldsymbol{v}) \\
& \forall q \in L^{2}(\Omega), \quad b(\boldsymbol{u}, q)=0
\end{aligned}
$$

where the linear form $\mathcal{L}(\cdot)$ is defined by

$$
\mathcal{L}(\boldsymbol{v})=\int_{\Omega} \boldsymbol{f}(\boldsymbol{x}) \cdot \boldsymbol{v}(\boldsymbol{x}) \mathrm{d} \boldsymbol{x}-\int_{\Gamma_{a P}}(\boldsymbol{v} \cdot \boldsymbol{n})(\boldsymbol{\tau}) p_{a}(\boldsymbol{\tau}) \mathrm{d} \boldsymbol{\tau}+\int_{\Gamma_{a F}} \boldsymbol{v}(\boldsymbol{\tau}) \cdot \mathbf{t}_{a}(\boldsymbol{\tau}) \mathrm{d} \boldsymbol{\tau}
$$

Note that, in this definition, we have used integrals for the sake of clarity, however they are most often replaced by duality pairings. Indeed, from now on, we make the following assumption on the five data

$$
k \in H^{-\frac{1}{2}}\left(\Gamma_{P}\right), \quad \boldsymbol{g} \in H^{\frac{1}{2}}\left(\Gamma_{F}\right)^{d}, \quad \boldsymbol{f} \in X_{0}(\Omega)^{\prime}, \quad p_{a} \in H_{00}^{\frac{1}{2}}\left(\Gamma_{a P}\right), \quad \mathbf{t}_{a} \in H^{-\frac{1}{2}}\left(\Gamma_{a F}\right)^{d},
$$

where $H^{-\frac{1}{2}}\left(\Gamma_{P}\right)$ and $H^{-\frac{1}{2}}\left(\Gamma_{a F}\right)$ stand for the dual spaces of $H^{\frac{1}{2}}\left(\Gamma_{P}\right)$ and $H^{\frac{1}{2}}\left(\Gamma_{a F}\right)$, respectively. With this choice, the boundary conditions (2.6) makes sense (see Rem. 2.1) and the form $\mathcal{L}(\cdot)$ is continuous on $X_{0}(\Omega)$.

Standard arguments lead to the equivalence of problems (1.1)-(1.5) and (2.6)-(2.7).

Proposition 2.2. Any smooth enough pair of functions $(\boldsymbol{u}, p)$ is a solution of problem (2.6)-(2.7) if and only if it is a solution of problem (1.1)-(1.5).

To prove the well-posedness of problem (2.6)-(2.7), we first construct a lifting of the boundary conditions (2.6).

Lemma 2.3. There exists a divergence-free function $\boldsymbol{u}_{b}$ in $X(\Omega)$ which satisfies

$$
\boldsymbol{u}_{b} \cdot \boldsymbol{n}=k \quad \text { on } \Gamma_{P} \quad \text { and } \quad \boldsymbol{u}_{b}=\boldsymbol{g} \quad \text { on } \Gamma_{F},
$$

and

$$
\left\|\boldsymbol{u}_{b}\right\|_{X(\Omega)} \leq c\left(\|k\|_{H^{-\frac{1}{2}}\left(\Gamma_{P}\right)}+\|\boldsymbol{g}\|_{H^{\frac{1}{2}}\left(\Gamma_{F}\right)^{d}}\right) .
$$


Proof. It is performed in three steps.

1) Let $\overline{\boldsymbol{g}}$ be an extension of $\boldsymbol{g}$ into $H^{\frac{1}{2}}\left(\partial \Omega_{F}\right)^{d}$. We introduce a fixed smooth vector field $\boldsymbol{\varphi}$ with support in $\Gamma$ and set

$$
\overline{\boldsymbol{g}}^{*}=\overline{\boldsymbol{g}}-\frac{\int_{\partial \Omega_{F}}(\overline{\boldsymbol{g}} \cdot \boldsymbol{n})(\boldsymbol{\tau}) \mathrm{d} \boldsymbol{\tau}}{\int_{\Gamma}(\boldsymbol{\varphi} \cdot \boldsymbol{n})(\boldsymbol{\tau}) \mathrm{d} \boldsymbol{\tau}} \varphi
$$

So the function $\overline{\boldsymbol{g}}^{*}$ belongs to $H^{\frac{1}{2}}\left(\partial \Omega_{F}\right)^{d}$ and satisfies

$$
\int_{\partial \Omega_{F}}\left(\overline{\boldsymbol{g}}^{*} \cdot \boldsymbol{n}\right)(\boldsymbol{\tau}) \mathrm{d} \boldsymbol{\tau}=0 \quad \text { and } \quad\left\|\overline{\boldsymbol{g}}^{*}\right\|_{H^{\frac{1}{2}}\left(\partial \Omega_{F}\right)^{d}} \leq c\|\boldsymbol{g}\|_{H^{\frac{1}{2}}\left(\Gamma_{F}\right)^{d}} .
$$

Thus, the Stokes problem

$$
\begin{cases}-\nu \Delta \boldsymbol{u}_{b F}+\operatorname{grad} p_{b F}=\mathbf{0} & \text { in } \Omega_{F}, \\ \operatorname{div} \boldsymbol{u}_{b F}=0 & \text { in } \Omega_{F} \\ \boldsymbol{u}_{b F}=\overline{\boldsymbol{g}}^{*} & \text { on } \partial \Omega_{F},\end{cases}
$$

has a solution in $H^{1}\left(\Omega_{F}\right) \times L^{2}\left(\Omega_{F}\right)$, which is unique up to an additive constant on the pressure [24], Chapter I, Theorem 5.1. Moreover, thanks to the previous inequality, this solution satisfies

$$
\left\|\boldsymbol{u}_{b F}\right\|_{H^{1}\left(\Omega_{F}\right)^{d}} \leq c\|\boldsymbol{g}\|_{H^{\frac{1}{2}}\left(\Gamma_{F}\right)^{d}}
$$

2) We now denote by $Y\left(\Omega_{P}\right)$ the space

$$
Y\left(\Omega_{P}\right)=\left\{\mu \in H^{1}\left(\Omega_{P}\right) ; \mu=0 \text { on } \Gamma_{a P}\right\}
$$

When $\Gamma_{a P}$ has a positive measure, we consider the problem:

Find $\lambda$ in $Y\left(\Omega_{P}\right)$ such that

$$
\forall \mu \in Y\left(\Omega_{P}\right), \quad \int_{\Omega_{P}}(\operatorname{grad} \lambda)(\boldsymbol{x}) \cdot(\operatorname{grad} \mu)(\boldsymbol{x})=\int_{\Gamma_{P}} k(\boldsymbol{\tau}) \mu(\boldsymbol{\tau}) \mathrm{d} \boldsymbol{\tau}+\int_{\Gamma}\left(\boldsymbol{u}_{b F} \cdot \boldsymbol{n}\right)(\boldsymbol{\tau}) \mu(\boldsymbol{\tau}) \mathrm{d} \boldsymbol{\tau} .
$$

This problem has a unique solution. Moreover the function $\boldsymbol{u}_{b P}=\operatorname{grad} \lambda$ is divergence-free on $\Omega_{P}$ (as follows by taking $\mu$ in $\mathscr{D}(\Omega)$ in the previous problem) and satisfies

$$
\boldsymbol{u}_{b P} \cdot \boldsymbol{n}=k \quad \text { on } \Gamma_{P} \quad \text { and } \quad \boldsymbol{u}_{b P} \cdot \boldsymbol{n}=\boldsymbol{u}_{b F} \cdot \boldsymbol{n} \quad \text { on } \Gamma,
$$

and

$$
\left\|\boldsymbol{u}_{b P}\right\|_{H\left(\operatorname{div}, \Omega_{P}\right)} \leq c\left(\|k\|_{H^{-\frac{1}{2}}\left(\Gamma_{P}\right)}+\|\boldsymbol{g}\|_{H^{\frac{1}{2}}\left(\Gamma_{F}\right)^{d}}\right) .
$$

3) When $\Gamma_{a P}$ has a zero measure, it follows from the definition of $\Gamma_{a P}$ and $\Gamma_{a F}$ that $\Gamma_{a F}$ has a positive measure. Thus, we introduce a further function $\boldsymbol{g}^{*}$ in $H^{\frac{1}{2}}(\Gamma)^{d}$ such that

$$
\int_{\Gamma}\left(\boldsymbol{g}^{*} \cdot \boldsymbol{n}\right)(\boldsymbol{\tau}) \mathrm{d} \boldsymbol{\tau}=-\int_{\Gamma_{P}} k(\boldsymbol{\tau}) \mathrm{d} \boldsymbol{\tau}
$$

and there exists a function $\bar{g}$ in $H^{\frac{1}{2}}\left(\partial \Omega_{F}\right)^{d}$ equal to $\boldsymbol{g}$ on $\Gamma_{F}$ and to $\boldsymbol{g}^{*}$ on $\Gamma$ (note that this requires some compatibility conditions between $\boldsymbol{g}$ and $\boldsymbol{g}^{*}$ on $\bar{\Gamma}_{F} \cap \bar{\Gamma}$ when this last set is not empty). By adding to $\overline{\boldsymbol{g}}$ a constant times a fixed smooth function now with support in $\Gamma_{a F}$, we construct a function $\overline{\boldsymbol{g}}^{*}$ in $H^{\frac{1}{2}}\left(\partial \Omega_{F}\right)$ which satisfies

$$
\int_{\partial \Omega_{F}}\left(\overline{\boldsymbol{g}}^{*} \cdot \boldsymbol{n}\right)(\boldsymbol{\tau}) \mathrm{d} \boldsymbol{\tau}=0
$$


Then the Stokes problem (2.12) with this modified function $\overline{\boldsymbol{g}}^{*}$ still admits a solution, and this solution satisfies

$$
\left\|\boldsymbol{u}_{b F}\right\|_{H^{1}\left(\Omega_{F}\right)^{d}} \leq c\left(\|k\|_{H^{-\frac{1}{2}}\left(\Gamma_{P}\right)}+\|\boldsymbol{g}\|_{H^{\frac{1}{2}}\left(\Gamma_{F}\right)^{d}}\right) .
$$

Next, since the function equal to $k$ on $\Gamma_{P}$ and to $\boldsymbol{u}_{b F} \cdot \boldsymbol{n}=\boldsymbol{g}^{*} \cdot \boldsymbol{n}$ on $\Gamma$ has a null integral on $\partial \Omega_{P}$, problem (2.14) admits a solution $\lambda$, unique up to an additive constant (note that $Y\left(\Omega_{P}\right)$ now coincides with $H^{1}\left(\Omega_{P}\right)$ ). The function $\boldsymbol{u}_{b P}=\operatorname{grad} \lambda$ is divergence-free on $\Omega_{P}$ and still satisfies (2.15) and (2.16).

To conclude, we observe from either (2.13) or (2.17) and (2.16) that the function $\boldsymbol{u}_{b}$ equal to $\boldsymbol{u}_{b P}$ on $\Omega_{P}$ and to $\boldsymbol{u}_{b F}$ on $\Omega_{F}$ satisfies all the desired properties.

Remark 2.4. Note that the first assumption in (2.9) could be replaced by the weaker one

$$
k \in H_{00}^{\frac{1}{2}}\left(\Gamma_{P}\right)^{\prime},
$$

see Remark 2.1. However, the previous proof does not work with only this assumption when, for instance, $\bar{\Gamma}_{P} \cap \bar{\Gamma}$ is not empty, see (2.14). So we do not handle this modified assumption since we have no direct application for it.

To go further, we set: $\boldsymbol{u}_{0}=\boldsymbol{u}-\boldsymbol{u}_{b}$, where $\boldsymbol{u}_{b}$ is the function exhibited in Lemma 2.3. We observe that problem (2.6)-(2.7) admits a solution if the following problem has one:

Find $\left(\boldsymbol{u}_{0}, p\right)$ in $X_{0}(\Omega) \times L^{2}(\Omega)$ such that

$$
\begin{aligned}
& \forall \boldsymbol{v} \in X_{0}(\Omega), \quad a\left(\boldsymbol{u}_{0}, \boldsymbol{v}\right)+b(\boldsymbol{v}, p)=-a\left(\boldsymbol{u}_{b}, \boldsymbol{v}\right)+\mathcal{L}(\boldsymbol{v}), \\
& \forall q \in L^{2}(\Omega), \quad b\left(\boldsymbol{u}_{0}, q\right)=0 .
\end{aligned}
$$

It is readily checked that the kernel

$$
V(\Omega)=\left\{\boldsymbol{v} \in X_{0}(\Omega) ; \forall q \in L^{2}(\Omega), b(\boldsymbol{v}, q)=0\right\},
$$

coincides with the space of functions in $X_{0}(\Omega)$ which are divergence-free on $\Omega$. We first check the ellipticity of the form $a(\cdot, \cdot)$ on $V(\Omega)$.

Lemma 2.5. Assume that

(i) either $\Gamma_{F}$ has a positive measure in $\partial \Omega_{F}$,

(ii) or the normal vector $\boldsymbol{n}(\boldsymbol{x})$ runs through a basis of $\mathbb{R}^{d}$ when $\boldsymbol{x}$ runs through $\Gamma$.

There exists a constant $\alpha_{*}>0$ such that the following ellipticity property holds

$$
\forall \boldsymbol{v} \in V(\Omega), \quad a(\boldsymbol{v}, \boldsymbol{v}) \geq \alpha_{*}\|\boldsymbol{v}\|_{X(\Omega)}^{2} .
$$

Proof. Let us observe that, for all $\boldsymbol{v}$ in $V(\Omega)$,

$$
a(\boldsymbol{v}, \boldsymbol{v}) \geq \min \{\alpha, \nu\}\left(\|\boldsymbol{v}\|_{L^{2}\left(\Omega_{P}\right)^{d}}^{2}+|\boldsymbol{v}|_{H^{1}\left(\Omega_{F}\right)^{d}}^{2}\right),
$$

and

$$
\|\boldsymbol{v}\|_{X(\Omega)}=\left(\|\boldsymbol{v}\|_{L^{2}\left(\Omega_{P}\right)^{d}}^{2}+|\boldsymbol{v}|_{H^{1}\left(\Omega_{F}\right)^{d}}^{2}+\|\boldsymbol{v}\|_{L^{2}\left(\Omega_{F}\right)^{d}}^{2}\right)^{\frac{1}{2}} .
$$

Let now $\boldsymbol{v}$ be a function in $V(\Omega)$ such that $\|\boldsymbol{v}\|_{L^{2}\left(\Omega_{P}\right)^{d}}$ and $|\boldsymbol{v}|_{H^{1}\left(\Omega_{F}\right)^{d}}$ are equal to zero. Thus, $\boldsymbol{v}$ is zero on $\Omega_{P}$ and is equal to a constant $\mathbf{c}$ on $\Omega_{F}$. When assumption (i) holds, it follows from the definition of $X_{0}(\Omega)$ that this constant is zero. When assumption (ii) holds, since $\boldsymbol{v}$ is zero on $\Omega_{P}, \mathbf{c} \cdot \boldsymbol{n}$ is zero on $\Gamma$ and, since $\boldsymbol{n}$ runs through a basis of $\mathbb{R}^{d}$, $\mathbf{c}$ is zero. Then $\boldsymbol{v}$ is zero on $\Omega$. Thanks to the Peetre-Tartar lemma [24], Chapter I, Theorem 2.1, it follows from this property, $(2.22)$ and the compactness of the imbedding of $H^{1}\left(\Omega_{F}\right)$ into $L^{2}\left(\Omega_{F}\right)$ that

$$
\forall \boldsymbol{v} \in V(\Omega), \quad\left(\|\boldsymbol{v}\|_{L^{2}\left(\Omega_{P}\right)^{d}}^{2}+|\boldsymbol{v}|_{H^{1}\left(\Omega_{F}\right)^{d}}^{2}\right)^{\frac{1}{2}} \geq c\|\boldsymbol{v}\|_{X(\Omega)} .
$$


This, combined with (2.21), gives the desired ellipticity property.

Lemma 2.6. There exists a constant $\beta>0$ such that the following inf-sup condition holds

$$
\forall q \in L^{2}(\Omega), \quad \sup _{\boldsymbol{v} \in X_{0}(\Omega)} \frac{b(\boldsymbol{v}, q)}{\|\boldsymbol{v}\|_{X(\Omega)}} \geq \beta\|q\|_{L^{2}(\Omega)} .
$$

Proof. Let $\Omega_{+}$be a rectangle $(d=2)$ or a rectangular parallelepiped $(d=3)$ such that $\Gamma_{+}=\Gamma_{a} \cap \partial \Omega_{+}$is contained in the interior of $\Gamma_{a}$ and has a positive measure. Then, the function $q_{+}$defined by

$$
q_{+}= \begin{cases}q & \text { on } \Omega, \\ -\frac{1}{\operatorname{meas}\left(\Omega_{+}\right)} \int_{\Omega} q(\boldsymbol{x}) \mathrm{d} \boldsymbol{x} & \text { on } \Omega_{+},\end{cases}
$$

belongs to $L^{2}\left(\Omega \cup \Omega_{+}\right)$and has a null integral on this domain. It thus follows from the standard inf-sup condition, see [24], Chapter I, Corollary 2.4, that there exists a function $\boldsymbol{v}_{+}$in $H_{0}^{1}\left(\Omega \cup \Gamma_{+} \cup \Omega_{+}\right)^{d}$ such that

$$
\operatorname{div} \boldsymbol{v}_{+}=-q_{+} \quad \text { and } \quad\left\|\boldsymbol{v}_{+}\right\|_{H^{1}\left(\Omega \cup \Gamma_{+} \cup \Omega_{+}\right)^{d}} \leq c\left\|q_{+}\right\|_{L^{2}\left(\Omega \cup \Gamma_{+} \cup \Omega_{+}\right)} .
$$

Taking $\boldsymbol{v}$ equal to the restriction of $\boldsymbol{v}_{+}$to $\Omega$ (which obviously belongs to $X_{0}(\Omega)$ ) leads to the desired inf-sup condition.

We are now in a position to prove the main result of this section. Note that, due to the mixed boundary conditions, no further assumption on the flux of the data is needed for the existence of a solution.

Theorem 2.7. If the assumptions of Lemma 2.5 hold, for any data $\left(k, \boldsymbol{g}, \boldsymbol{f}, p_{a}, \mathbf{t}_{a}\right)$ satisfying (2.9), problem (2.6)-(2.7) has a unique solution $(\boldsymbol{u}, p)$ in $X(\Omega) \times L^{2}(\Omega)$. Moreover this solution satisfies

$$
\|\boldsymbol{u}\|_{X(\Omega)}+\|p\|_{L^{2}(\Omega)} \leq c\left(\|k\|_{H^{-\frac{1}{2}}\left(\Gamma_{P}\right)}+\|\boldsymbol{g}\|_{H^{\frac{1}{2}\left(\Gamma_{F}\right)^{d}}}+\|\boldsymbol{f}\|_{X_{0}(\Omega)^{\prime}}+\left\|p_{a}\right\|_{H_{00}^{\frac{1}{2}\left(\Gamma_{a P}\right)}}+\left\|\mathbf{t}_{a}\right\|_{H^{-\frac{1}{2}}\left(\Gamma_{a F}\right)^{d}}\right) .
$$

Proof. It follows from Lemmas 2.5 and 2.6, see [24], Chapter I, Theorem 4.1, that problem (2.18) has a unique solution $\left(\boldsymbol{u}_{0}, p\right)$ in $X_{0}(\Omega) \times L^{2}(\Omega)$ and that this solution satisfies

$$
\left\|\boldsymbol{u}_{0}\right\|_{X(\Omega)}+\|p\|_{L^{2}(\Omega)} \leq c\left(\left\|\boldsymbol{u}_{b}\right\|_{X(\Omega)}+\|\boldsymbol{f}\|_{X_{0}(\Omega)^{\prime}}+\left\|p_{a}\right\|_{H_{00}^{\frac{1}{2}\left(\Gamma_{a P}\right)}}+\left\|\mathbf{t}_{a}\right\|_{H^{-\frac{1}{2}}\left(\Gamma_{a F}\right)^{d}}\right) .
$$

Then, the pair $\left(\boldsymbol{u}=\boldsymbol{u}_{0}+\boldsymbol{u}_{b}, p\right)$ is a solution of problem (2.6)-(2.7), and estimate (2.24) is a consequence of (2.25) and (2.11). On the other hand, let $\left(\boldsymbol{u}_{1}, p_{1}\right)$ and $\left(\boldsymbol{u}_{2}, p_{2}\right)$ be two solutions of problem (2.6)-(2.7). Then, the difference $\left(\boldsymbol{u}_{1}-\boldsymbol{u}_{2}, p_{1}-p_{2}\right)$ is a solution of problem (2.18) with data $\boldsymbol{u}_{b}, \boldsymbol{f}, p_{a}$ and $\mathbf{t}_{a}$ equal to zero. Thus, it follows from (2.25) that it is zero. So the solution of problem (2.6)-(2.7) is unique.

From now on, we assume that the non restrictive assumptions of Lemma 2.5 hold. We conclude with some regularity properties of the solution $(\boldsymbol{u}, p)$.

Proposition 2.8. Let us assume that the five data satisfy

$$
k \in H^{\frac{1}{2}}\left(\Gamma_{P}\right), \quad \boldsymbol{g} \in H^{\frac{3}{2}}\left(\Gamma_{F}\right)^{d}, \quad \boldsymbol{f} \in H^{1}(\Omega)^{d}, \quad p_{a} \in H^{\frac{3}{2}}\left(\Gamma_{a P}\right), \quad \mathbf{t}_{a} \in H^{\frac{1}{2}}\left(\Gamma_{a F}\right)^{d} .
$$

Then, the restriction $\left(\boldsymbol{u}_{\mid \Omega_{P}}, p_{\mid \Omega_{P}}\right)$ of the solution $(\boldsymbol{u}, p)$ of problem (2.6)-(2.7) to $\Omega_{P}$ belongs to the space $H^{s_{P}}\left(\Omega_{P}\right)^{d} \times H^{s_{P}+1}\left(\Omega_{P}\right)$ for a real number $s_{P}>0$ given by

- $s_{P}=1 / 4$ if $\Omega_{P}$ is a polygon $(d=2)$;

- $s_{P}=1 / 2$ if $\Gamma_{a P}$ is empty or if $\Omega_{P}$ is a polygon or a polyhedron and there exists a convex neighbourhood in $\Omega_{P}$ of $\left(\bar{\Gamma}_{P} \cup \bar{\Gamma}\right) \cap \Gamma_{a P}$; 
- $s_{P}<1$ if $\Gamma_{a P}$ is empty and $\Omega_{P}$ is a convex polygon or polyhedron or has a $\mathscr{C}^{1,1}$-boundary.

The restriction $\left(\boldsymbol{u}_{\mid \Omega_{F}}, p_{\mid \Omega_{F}}\right)$ of the solution $(\boldsymbol{u}, p)$ of problem (2.6)-(2.7) to $\Omega_{F}$ belongs to the space $H^{s_{F}+1}\left(\Omega_{F}\right)^{d} \times H^{s_{F}}\left(\Omega_{F}\right)$ for a real number $s_{F}>0$ given by

- $s_{F}=1 / 4$ if $\Omega_{F}$ is a polygon $(d=2)$;

- $s_{F}=1 / 2$ if $\Gamma_{F}$ is empty or if $\Omega_{F}$ is a polygon $(d=2)$ and there exists a convex neighbourhood in $\Omega_{F}$ of $\left(\bar{\Gamma}_{a F} \cup \bar{\Gamma}\right) \cap \Gamma_{F}$;

- $s_{F}<1$ if $\Gamma_{F}$ is empty and $\Omega_{F}$ is a convex polygon or polyhedron or has a $\mathscr{C}^{1,1}$-boundary.

Proof. We check successively the two assertions.

1) The function $p_{\mid \Omega_{P}}$ is a solution of the Poisson equation with mixed boundary conditions

$$
\begin{cases}-\Delta p=-\operatorname{div} \boldsymbol{f} & \text { in } \Omega_{P} \\ p=p_{a} & \text { on } \Gamma_{a P} \\ \partial_{n} p=\boldsymbol{f} \cdot \boldsymbol{n}-\alpha k & \text { on } \Gamma_{P} \\ \partial_{n} p=\boldsymbol{f} \cdot \boldsymbol{n}-\alpha \boldsymbol{u}_{\mid \Omega_{F}} \cdot \boldsymbol{n} & \text { on } \Gamma\end{cases}
$$

Moreover, since $\boldsymbol{u}_{\mid \Omega_{F}}$ belongs to $H^{1}(\Omega)^{d}$, its normal trace $\boldsymbol{u}_{\mid \Omega_{F}} \cdot \boldsymbol{n}$ belongs to $H^{\frac{1}{2}}(\Gamma)$. The desired regularity of $p_{\mid \Omega_{P}}$ is easily derived from [26], Theorems 2.2.2.3 and 3.2.1.2, or [19], Section 3, thanks to appropriate Sobolev imbeddings. The regularity of $\boldsymbol{u}_{\mid \Omega_{P}}$ then follows from the first line in (1.1).

2) The pair $\left(\boldsymbol{u}_{\mid \Omega_{F}}, p_{\mid \Omega_{F}}\right)$ is a solution of the Stokes problem with mixed boundary conditions

$$
\begin{cases}-\nu \Delta \boldsymbol{u}+\operatorname{grad} p=\boldsymbol{f} & \text { in } \Omega_{F}, \\ \operatorname{div} \boldsymbol{u}=0 & \text { in } \Omega_{F}, \\ \boldsymbol{u}=\boldsymbol{g} & \text { on } \Gamma_{F} \\ \nu \partial_{n} \boldsymbol{u}-p \boldsymbol{n}=\mathbf{t}_{a} & \text { on } \Gamma_{a F} \\ \nu \partial_{n} \boldsymbol{u}-p \boldsymbol{n}=-p_{\mid \Omega_{P}} \boldsymbol{n} & \text { on } \Gamma\end{cases}
$$

It can also be noted from part 1) of the proof that $p_{\mid \Omega_{P}} \boldsymbol{n}$ belongs at least to $H^{\frac{1}{2}}(\Gamma)^{d}$. So the desired results follow from [32].

Assumption (2.26) is too strong for most results of Proposition 2.8, and we only make it for simplicity. Moreover the norms of $\left(\boldsymbol{u}_{\mid \Omega_{P}}, p_{\mid \Omega_{P}}\right)$ in $H^{s_{P}}\left(\Omega_{P}\right)^{d} \times H^{s_{P}+1}\left(\Omega_{P}\right)$ and of $\left(\boldsymbol{u}_{\mid \Omega_{F}}, p_{\mid \Omega_{F}}\right)$ in $H^{s_{F}+1}\left(\Omega_{F}\right)^{d} \times H^{s_{F}}\left(\Omega_{F}\right)$ are bounded as a function of weaker norms of the data. Note also that compatibility conditions on the data at the intersections of different parts of the boundaries should be made to obtain higher regularity, i.e. to break the restrictions $s_{P}<1$ and $s_{F}<1$. Similar results hold in other situations that we do not consider in this work (for instance, when $\Gamma_{a F}$ is empty).

\section{The Discrete PROBLEM AND ITS WELL-POSEDNESS}

The mortar finite element discretization relies on the partition of $\Omega$ into $\Omega_{P}$ and $\Omega_{F}$. Indeed, even if some further partitions could be introduced to handle anisotropic domains for instance, we do not consider them in this work. Let $\left(\mathcal{T}_{h}^{P}\right)_{h_{P}}$ and $\left(\mathcal{T}_{h}^{F}\right)_{h_{F}}$ be regular families of triangulations of $\Omega_{P}$ and $\Omega_{F}$, respectively, by closed triangles $(d=2)$ or tetrahedra $(d=3)$, in the usual sense that:

- For each $h_{P}, \bar{\Omega}_{P}$ is the union of all elements of $\mathcal{T}_{h}^{P}$ and, for each $h_{F}, \bar{\Omega}_{F}$ is the union of all elements of $\mathcal{T}_{h}^{F}$.

- The intersection of two different elements of $\mathcal{T}_{h}^{P}$, if not empty, is a vertex or a whole edge or a whole face of both of them, and the same property holds for the intersection of two different elements of $\mathcal{T}_{h}^{F}$.

- The ratio of the diameter $h_{K}$ of any element $K$ of $\mathcal{T}_{h}^{P}$ or of $\mathcal{T}_{h}^{F}$ to the diameter of its inscribed circle or sphere is smaller than a constant $\sigma$ independent of $h_{P}$ and $h_{F}$. 
As usual, $h_{P}$ stands for the maximum of the diameters of the elements of $\mathcal{T}_{h}^{P}$ and $h_{F}$ for the maximum of the diameters of the elements of $\mathcal{T}_{h}^{F}$. From now on, $c, c^{\prime}, \ldots$ denote for generic constants that may vary from one line to the next but are always independent of $h_{P}$ and $h_{F}$. We make the further standard and non restrictive assumptions.

Assumption 3.1. The intersection of each element $K$ of $\mathcal{T}_{h}^{P}$ with either $\bar{\Gamma}_{a P}$ or $\bar{\Gamma}_{P}$ or $\bar{\Gamma}$, if not empty, is a vertex or a whole edge or a whole face of $K$. The intersection of each element $K$ of $\mathcal{T}_{h}^{F}$ with either $\bar{\Gamma}_{a F}$ or $\bar{\Gamma}_{F}$ or $\bar{\Gamma}$, if not empty, is a vertex or a whole edge or a whole face of $K$.

It must be noted that, up to now, no assumption is made on the intersection of the elements of $\mathcal{T}_{h}^{P}$ and $\mathcal{T}_{h}^{F}$. So the $K \cap \Gamma, K \in \mathcal{T}_{h}^{P}$, and the $K \cap \Gamma, K \in \mathcal{T}_{h}^{F}$, form two independent triangulations of $\Gamma$, that we denote by $\mathcal{E}_{h}^{P, \Gamma}$ and $\mathcal{E}_{h}^{F, \Gamma}$, respectively. However, we are led to make another assumption.

Assumption 3.2. For any element $K$ of $\mathcal{T}_{h}^{F}$, the number of elements $K^{\prime}$ of $\mathcal{T}_{h}^{P}$ such that $\partial K \cap \partial K^{\prime}$ has a positive $(d-1)$-measure is bounded independently of $K, h_{P}$ and $h_{F}$.

We now define the local discrete spaces. For the reasons already explained in the introduction, the space of discrete velocities in $\Omega_{P}$ is constructed from the Raviart-Thomas finite element [34], which leads to the following definition

$$
X_{h}^{P}=\left\{\boldsymbol{v}_{h} \in H\left(\operatorname{div}, \Omega_{P}\right) ; \forall K \in \mathcal{T}_{h}^{P}, \boldsymbol{v}_{h \mid K} \in \mathcal{P}_{R T}(K)\right\},
$$

where $\mathcal{P}_{R T}(K)$ stands for the space of restrictions to $K$ of polynomials of the form $\boldsymbol{a}+b \boldsymbol{x}, \boldsymbol{a} \in \mathbb{R}^{d}$ and $b \in \mathbb{R}$. We also introduce the space

$$
X_{0 h}^{P}=\left\{\boldsymbol{v}_{h} \in X_{h}^{P} ; \boldsymbol{v}_{h} \cdot \boldsymbol{n}=0 \text { on } \Gamma_{P}\right\} .
$$

Similarly, on $\Omega_{F}$, we consider the space related to the Bernardi-Raugel element [6], i.e.

$$
X_{h}^{F}=\left\{\boldsymbol{v}_{h} \in H^{1}\left(\Omega_{F}\right)^{d} ; \forall K \in \mathcal{T}_{h}^{F}, \boldsymbol{v}_{h \mid K} \in \mathcal{P}_{B R}(K)\right\},
$$

where $\mathcal{P}_{B R}(K)$ stands for the space spanned by the restrictions to $K$ of affine functions on $\mathbb{R}^{d}$ with values in $\mathbb{R}^{d}$ and the $d+1$ normal bubble functions $\psi_{e} \boldsymbol{n}_{e}$ (for each edge $(d=2)$ or face $(d=3) e$ of $K, \psi_{e}$ denotes the bubble function on $e$ equal to the product of the barycentric coordinates associated with the endpoints or vertices of $e$ and $\boldsymbol{n}_{e}$ stands for the unit outward normal vector on $e$ ). We also need the space

$$
X_{0 h}^{F}=\left\{\boldsymbol{v}_{h} \in X_{h}^{F} ; \boldsymbol{v}_{h}=\mathbf{0} \text { on } \Gamma_{F}\right\} .
$$

Let now $h$ denote the discretization parameter, here equal to the pair $\left(h_{P}, h_{F}\right)$, and let $\mathcal{T}_{h}$ stand for the union of $\mathcal{T}_{h}^{P}$ and $\mathcal{T}_{h}^{F}$. We define the discrete space of pressures as

$$
\mathbb{M}_{h}=\left\{q_{h} \in L^{2}(\Omega) ; \forall K \in \mathcal{T}_{h}, q_{h \mid K} \in \mathcal{P}_{0}(K)\right\},
$$

where $\mathcal{P}_{0}(K)$ is the space of constant functions on $K$.

Remark 3.3. Other choices of finite elements are possible. Indeed, the Raviart-Thomas element is the simplest div-conforming element and the Bernardi-Raugel element is the less expensive $H^{1}$-conforming finite element for the Stokes problem. In dimension $d=2$, piecewise quadratic velocities can also be used on $\Omega_{F}$ and in dimension $d=3, \mathcal{P}_{B R}(K)$ can be replaced by the space spanned by affine functions and the $\psi_{e}$, up to the power $d$.

The skeleton of the decomposition is now the interface $\Gamma$. As standard for the mortar element method, see [7] and [11], the construction of the global space of velocities relies on the fact that the matching conditions are enforced via the orthogonality to functions defined on $\mathcal{T}_{h}^{P}$ or $\mathcal{T}_{h}^{F}$. Since these matching conditions only deal with the normal trace of the velocity, we have decided to make the choice proposed in [12], Section 3, which is 
more naturally associated with functions defined on $\mathcal{T}_{h}^{P}$, i.e. we define the space

$$
\mathbb{W}_{h}=\left\{\varphi_{h} \in L^{2}(\Gamma) ; \forall e \in \mathcal{E}_{h}^{P, \Gamma}, \varphi_{h \mid e} \in \mathcal{P}_{0}(e)\right\}
$$

with obvious definition for $\mathcal{P}_{0}(e)$.

The global spaces of velocities are then the spaces $\mathbb{X}_{h}$ and $\mathbb{X}_{0 h}$ of functions $\boldsymbol{v}_{h}$ such that

- their restrictions $\boldsymbol{v}_{h \mid \Omega_{P}}$ to $\Omega_{P}$ belong to $X_{h}^{P}$ and $X_{0 h}^{P}$, respectively;

- their restrictions $\boldsymbol{v}_{h \mid \Omega_{F}}$ to $\Omega_{F}$ belong to $X_{h}^{F}$ and $X_{0 h}^{F}$, respectively;

- the following matching conditions hold on $\Gamma$

$$
\forall \varphi_{h} \in \mathbb{W}_{h}, \quad \int_{\Gamma}\left(\left(\boldsymbol{v}_{h \mid \Omega_{P}}-\boldsymbol{v}_{h \mid \Omega_{F}}\right) \cdot \boldsymbol{n}\right)(\boldsymbol{\tau}) \varphi_{h}(\boldsymbol{\tau}) \mathrm{d} \boldsymbol{\tau}=0
$$

where $\boldsymbol{\tau}$ stands for the tangential coordinate(s) on $\Gamma$. Note that these conditions are not sufficient to enforce the continuity of $\boldsymbol{v}_{h} \cdot \boldsymbol{n}$ through $\Gamma$, so that the discretization is nonconforming: For instance, $\mathbb{X}_{h}$ is not contained in $H(\operatorname{div}, \Omega)$. However, the spaces $\mathbb{X}_{h}$ and $\mathbb{X}_{0 h}$ are still equipped with the norm $\|\cdot\|_{X(\Omega)}$.

Remark 3.4. In the implementation of the discrete problem, the matching conditions (3.7) are handled via the introduction of a Lagrange multiplier, as usual for the mortar method. We refer to [5] for the first analysis of this algorithm and to [11], Section 4, for another way of treating these conditions.

To discretize the essential boundary conditions that appear in (2.6), we now define the approximations of the data $k$ and $\boldsymbol{g}$ that we use in this work. We denote by $k_{h}$ the piecewise constant approximation of $k$ defined by

$$
\forall K \in \mathcal{T}_{h}^{P} / \operatorname{meas}\left(K \cap \Gamma_{P}\right)>0, \quad k_{h \mid K \cap \Gamma_{P}}=\frac{1}{\operatorname{meas}\left(K \cap \Gamma_{P}\right)} \int_{K \cap \Gamma_{P}} k(\boldsymbol{\tau}) \mathrm{d} \boldsymbol{\tau} .
$$

Note that this choice requires that $k$ belongs to $H^{-\sigma}(\Omega), \sigma<\frac{1}{2}$. We also introduce an approximation of $\boldsymbol{g}$ : When assuming that $\boldsymbol{g}$ is continuous on $\bar{\Gamma}_{F}$ (which is slightly stronger than the hypothesis made in (2.9)), the function $\boldsymbol{g}_{h}$

- belongs to the trace space of $X_{h}^{F}$;

- for each $K$ in $\mathcal{T}_{h}^{F}$, is equal to $\boldsymbol{g}(\boldsymbol{a})$ at each endpoint or vertex $\boldsymbol{a}$ of $K \cap \Gamma_{F}$;

- and satisfies

$$
\int_{K \cap \Gamma_{F}}\left(\boldsymbol{g}_{h} \cdot \boldsymbol{n}\right)(\boldsymbol{\tau}) \mathrm{d} \boldsymbol{\tau}=\int_{K \cap \Gamma_{F}}(\boldsymbol{g} \cdot \boldsymbol{n})(\boldsymbol{\tau}) \mathrm{d} \boldsymbol{\tau} .
$$

Indeed, these conditions define $k_{h}$ and $\boldsymbol{g}_{h}$ in a unique way, as follows from [34], Remark 3, and [6], Lemma II.1.

We are now in a position to write the discrete problem, which is constructed by the Galerkin method from $(2.7)$. It reads:

Find $\left(\boldsymbol{u}_{h}, p_{h}\right)$ in $\mathbb{X}_{h} \times \mathbb{M}_{h}$ such that

$$
\boldsymbol{u}_{h} \cdot \boldsymbol{n}=k_{h} \quad \text { on } \Gamma_{P} \quad \text { and } \quad \boldsymbol{u}_{h}=\boldsymbol{g}_{h} \quad \text { on } \Gamma_{F},
$$

and that

$$
\begin{aligned}
& \forall \boldsymbol{v}_{h} \in \mathbb{X}_{0 h}, \quad a\left(\boldsymbol{u}_{h}, \boldsymbol{v}_{h}\right)+\tilde{b}\left(\boldsymbol{v}_{h}, p_{h}\right)=\mathcal{L}\left(\boldsymbol{v}_{h}\right), \\
& \forall q_{h} \in \mathbb{M}_{h}, \quad \tilde{b}\left(\boldsymbol{u}_{h}, q_{h}\right)=0
\end{aligned}
$$

where the bilinear form $\tilde{b}(\cdot, \cdot)$ is defined by

$$
\tilde{b}(\boldsymbol{v}, q)=-\int_{\Omega_{P}}\left(\operatorname{div} \boldsymbol{v}_{\mid \Omega_{P}}\right)(\boldsymbol{x}) q(\boldsymbol{x}) \mathrm{d} \boldsymbol{x}-\int_{\Omega_{F}}\left(\operatorname{div} \boldsymbol{v}_{\mid \Omega_{F}}\right)(\boldsymbol{x}) q(\boldsymbol{x}) \mathrm{d} \boldsymbol{x} .
$$


The introduction of this modified form is due to the nonconformity of the discretization, and it is readily checked that it coincides with $b(\cdot, \cdot)$ on $H(\operatorname{div}, \Omega) \times L^{2}(\Omega)$.

As in the continuous case, to prove the well-posedness of problem (3.9)-(3.10), we first construct a lifting of the boundary conditions (3.9). It requires the Raviart-Thomas operator $\Pi_{h}^{R T}$, see [34], Section 3, and also [31], Section 1.3, for its three-dimensional analogue: For any smooth enough function $\boldsymbol{v}$ on $\Omega_{P}, \Pi_{h}^{R T} \boldsymbol{v}$ belongs to $X_{h}^{P}$ and satisfies on all edges $(d=2)$ or faces $(d=3) e$ of elements of $\mathcal{T}_{h}^{P}$,

$$
\int_{e}\left(\Pi_{h}^{R T} \boldsymbol{v} \cdot \boldsymbol{n}\right)(\boldsymbol{\tau}) \mathrm{d} \boldsymbol{\tau}=\int_{e}(\boldsymbol{v} \cdot \boldsymbol{n})(\boldsymbol{\tau}) \mathrm{d} \boldsymbol{\tau}
$$

The fact that these equations define the operator $\Pi_{h}^{R T}$ in a unique way and its main properties are proved in [34], Theorem 3, in the two-dimensional case. Moreover, this operator preserves the nullity of the normal trace on $\Gamma_{P}$ (this requires Assumption 3.1). Similarly, we introduce another operator that we call Bernardi-Raugel operator and denote by $\Pi_{h}^{B R}$ : For any continuous function $\boldsymbol{v}$ on $\bar{\Omega}_{F}, \Pi_{h}^{B R} \boldsymbol{v}$ belongs to $X_{h}^{F}$, is equal to $\boldsymbol{v}(\boldsymbol{a})$ at any vertex $\boldsymbol{a}$ of the elements of $\mathcal{T}_{h}^{F}$ and satisfies on all edges $(d=2)$ or faces $(d=3) e$ of elements of $\mathcal{T}_{h}^{F}$,

$$
\int_{e}\left(\Pi_{h}^{B R} \boldsymbol{v} \cdot \boldsymbol{n}\right)(\boldsymbol{\tau}) \mathrm{d} \boldsymbol{\tau}=\int_{e}(\boldsymbol{v} \cdot \boldsymbol{n})(\boldsymbol{\tau}) \mathrm{d} \boldsymbol{\tau}
$$

This defines the operator $\Pi_{h}^{B R}$ in a unique way, see [6], Lemma II.1.

We now establish some properties of the operator $\Pi_{h}^{R T}$. We refer to [25], Appendix, for their proof in the two-dimensional case and for quadrilateral finite elements and to [15], Section III.3, for additional results. It requires the Piola transform $\mathcal{A}_{K}$, defined as follows, see [24], Chapter III, formula (4.63): For any element $K$ of $\mathcal{T}_{h}^{P}$, denoting by $F_{K}$ one of the affine mappings which maps the reference triangle or tetrahedron $\hat{K}$ onto $K$ and by $B_{K}$ the Jacobian matrix of $F_{K}$, we associate with any vector field $\hat{\boldsymbol{v}}$ defined on $\hat{K}$ the vector field $\boldsymbol{v}=\mathcal{A}_{K} \hat{\boldsymbol{v}}$ defined on $K$ by the formula

$$
\left(\mathcal{A}_{K} \hat{\boldsymbol{v}}\right) \circ F_{K}=\frac{1}{\left|\operatorname{det} B_{K}\right|} B_{K} \hat{\boldsymbol{v}}
$$

We recall two properties of this transform, valid for all smooth enough functions $\boldsymbol{v}$ and $\varphi$

$$
\begin{gathered}
(\operatorname{div} \boldsymbol{v}) \circ F_{K}=\frac{1}{\left|\operatorname{det} B_{K}\right|} \operatorname{div}\left(\mathcal{A}_{K}^{-1} \boldsymbol{v}\right) \\
\int_{\partial K}(\boldsymbol{v} \cdot \boldsymbol{n})(\boldsymbol{\tau}) \varphi(\boldsymbol{\tau}) \mathrm{d} \boldsymbol{\tau}=\int_{\partial \hat{K}}\left(\mathcal{A}_{K}^{-1} \boldsymbol{v} \cdot \hat{\boldsymbol{n}}\right)(\hat{\boldsymbol{\tau}})\left(\varphi \circ F_{K}\right)(\hat{\boldsymbol{\tau}}) \mathrm{d} \hat{\boldsymbol{\tau}}
\end{gathered}
$$

where $\boldsymbol{n}$ and $\hat{\boldsymbol{n}}$ stand for the unit outward normal vectors to $K$ and $\hat{K}$, respectively. We also introduce the basis functions associated with the space $X_{h}^{P}$ : If $\mathcal{E}_{h}^{P}$ denotes the set of edges $(d=2)$ or faces $(d=3)$ of elements of $\mathcal{T}_{h}^{P}$, with each $e$ in $\mathcal{E}_{h}^{P}$, we associate the function $\varphi_{e}$ in $X_{h}^{P}$ such that

$$
\int_{e}\left(\boldsymbol{\varphi}_{e} \cdot \boldsymbol{n}\right)(\boldsymbol{\tau}) \mathrm{d} \boldsymbol{\tau}=1 \quad \text { and } \quad \forall e^{\prime} \in \mathcal{E}_{h}^{P}, e^{\prime} \neq e, \quad \int_{e^{\prime}}\left(\boldsymbol{\varphi}_{e} \cdot \boldsymbol{n}\right)(\boldsymbol{\tau}) \mathrm{d} \boldsymbol{\tau}=0 .
$$

The $\boldsymbol{\varphi}_{e}, e \in \mathcal{E}_{h}^{P}$, form a basis of $X_{h}^{P}$. Moreover, it is readily checked that each $\boldsymbol{\varphi}_{e} \cdot \boldsymbol{n}$ is piecewise constant, equal to $\frac{1}{\operatorname{meas}(e)}$ on $e$ and to zero on all $e^{\prime} \neq e$.

Lemma 3.5. The following property holds for any $K$ in $\mathcal{T}_{h}^{P}$ and any $\boldsymbol{v}$ in $H\left(\operatorname{div}, \Omega_{P}\right)$,

$$
\left\|\operatorname{div} \Pi_{h}^{R T} \boldsymbol{v}\right\|_{L^{2}(K)} \leq\|\operatorname{div} \boldsymbol{v}\|_{L^{2}(K)} .
$$


The following property holds for any $K$ in $\mathcal{T}_{h}^{P}$ and any $\boldsymbol{v}$ in $H\left(\operatorname{div}, \Omega_{P}\right) \cap H^{s}\left(\Omega_{P}\right)^{d}, 0<s<\frac{1}{2}$,

$$
\left\|\Pi_{h}^{R T} \boldsymbol{v}\right\|_{L^{2}(K)^{d}} \leq c\left(\|\boldsymbol{v}\|_{L^{2}(K)^{d}}+h_{K}^{s}|\boldsymbol{v}|_{H^{s}(K)^{d}}+h_{K}\|\operatorname{div} \boldsymbol{v}\|_{L^{2}(K)}\right) .
$$

Proof. We check successively the two assertions of the lemma.

1) Since the divergence of all functions in $X_{h}^{P}$ is constant on each element $K$ of $\mathcal{T}_{h}^{P}$, we have

$$
\begin{aligned}
\left\|\operatorname{div} \Pi_{h}^{R T} \boldsymbol{v}\right\|_{L^{2}(K)}^{2} & =\left.\left(\operatorname{div} \Pi_{h}^{R T} \boldsymbol{v}\right)\right|_{K} \int_{K}\left(\operatorname{div} \Pi_{h}^{R T} \boldsymbol{v}\right)(\boldsymbol{x}) \mathrm{d} \boldsymbol{x} \\
& =\left.\left(\operatorname{div} \Pi_{h}^{R T} \boldsymbol{v}\right)\right|_{K} \int_{\partial K}\left(\Pi_{h}^{R T} \boldsymbol{v} \cdot \boldsymbol{n}\right)(\boldsymbol{\tau}) \mathrm{d} \boldsymbol{\tau} .
\end{aligned}
$$

It follows from the definition (3.12) of $\Pi_{h}^{R T}$ that

$$
\left\|\operatorname{div} \Pi_{h}^{R T} \boldsymbol{v}\right\|_{L^{2}(K)}^{2}=\left.\left(\operatorname{div} \Pi_{h}^{R T} \boldsymbol{v}\right)\right|_{K} \int_{\partial K}(\boldsymbol{v} \cdot \boldsymbol{n})(\boldsymbol{\tau}) \mathrm{d} \boldsymbol{\tau}=\int_{K}\left(\operatorname{div} \Pi_{h}^{R T} \boldsymbol{v}\right)(\operatorname{div} \boldsymbol{v})(\boldsymbol{x}) \mathrm{d} \boldsymbol{x},
$$

so that using a Cauchy-Schwarz inequality yields (3.18).

2) Denoting by $\overline{\mathcal{E}}_{K}$ the set of edges $(d=2)$ or faces $(d=3)$ of $K$, we have from (3.12)

$$
\left.\left(\Pi_{h}^{R T} \boldsymbol{v}\right)\right|_{K}=\sum_{e \in \overline{\mathcal{E}}_{K}}\left(\int_{e}(\boldsymbol{v} \cdot \boldsymbol{n})(\boldsymbol{\tau}) \mathrm{d} \boldsymbol{\tau}\right) \boldsymbol{\varphi}_{e}
$$

so that

$$
\left\|\Pi_{h}^{R T} \boldsymbol{v}\right\|_{L^{2}(K)^{d}} \leq \sum_{e \in \overline{\mathcal{E}}_{K}}\left|\int_{e}(\boldsymbol{v} \cdot \boldsymbol{n})(\boldsymbol{\tau}) \mathrm{d} \boldsymbol{\tau}\right|\left\|\boldsymbol{\varphi}_{e}\right\|_{L^{2}(K)^{d}} .
$$

When setting $\hat{e}=F_{K}^{-1}(e)$, it follows from (3.16) and (3.17) that the function $\widehat{\varphi_{e}}=\mathcal{A}_{K}^{-1} \boldsymbol{\varphi}_{e}$ is such that

$$
\int_{\hat{e}}\left(\widehat{\boldsymbol{\varphi}_{e}} \cdot \hat{\boldsymbol{n}}\right)(\hat{\boldsymbol{\tau}}) \mathrm{d} \hat{\boldsymbol{\tau}}=1 \quad \text { and } \quad \forall \hat{e}^{\prime} \in \overline{\mathcal{E}}_{\hat{K}}, \hat{e}^{\prime} \neq \hat{e}, \quad \int_{\hat{e}^{\prime}}\left(\widehat{\boldsymbol{\varphi}}_{e} \cdot \hat{\boldsymbol{n}}\right)(\hat{\boldsymbol{\tau}}) \mathrm{d} \hat{\boldsymbol{\tau}}=0
$$

so that $\left\|\widehat{\varphi_{e}}\right\|_{L^{2}(\hat{K})^{d}}$ is bounded independently of $K$. Thus, standard arguments relying on (3.14) give

$$
\left\|\varphi_{e}\right\|_{L^{2}(K)^{d}} \leq c h_{K}^{1-\frac{d}{2}}
$$

On the other hand, denoting by $\chi_{e}$ the function equal to 1 on $e$ and to 0 on $\partial K \backslash e$, by $\widehat{\chi_{e}}$ the function $\chi_{e} \circ F_{K}$ and by $\overline{\bar{\chi}_{e}}$ a lifting of $\widehat{\chi_{e}}$ to $\hat{K}$, we have from (3.16)

$$
\begin{aligned}
\int_{e}(\boldsymbol{v} \cdot \boldsymbol{n})(\boldsymbol{\tau}) \mathrm{d} \boldsymbol{\tau} & =\int_{\partial \hat{K}}\left(\mathcal{A}_{K}^{-1} \boldsymbol{v} \cdot \hat{\boldsymbol{n}}\right)(\hat{\boldsymbol{\tau}}) \widehat{\chi_{e}}(\hat{\boldsymbol{\tau}}) \mathrm{d} \hat{\boldsymbol{\tau}} \\
& =\int_{\hat{K}}\left(\mathcal{A}_{K}^{-1} \boldsymbol{v}\right)(\hat{\boldsymbol{x}}) \cdot\left(\operatorname{grad} \overline{\widehat{\chi_{e}}}\right)(\hat{\boldsymbol{x}}) \mathrm{d} \hat{\boldsymbol{x}}+\int_{\hat{K}}\left(\operatorname{div}\left(\mathcal{A}_{K}^{-1} \boldsymbol{v}\right)\right)(\hat{\boldsymbol{x}}) \overline{\widehat{\chi_{e}}}(\hat{\boldsymbol{x}}) \mathrm{d} \hat{\boldsymbol{x}} .
\end{aligned}
$$

Note however that, since $\widehat{\chi_{e}}$ only belongs to $H^{r}(\partial \hat{K})$ for all $r<\frac{1}{2},\left(\operatorname{grad} \overline{\widehat{\chi_{e}}}\right)(\hat{\boldsymbol{x}})$ only belongs to $H^{r-\frac{1}{2}}(\hat{K})$ and that the first integral in the second line of the previous equation must be replaced by a duality pairing. Then, choosing $r$ such that $\frac{1}{2}-r=s$ yields

$$
\left|\int_{e}(\boldsymbol{v} \cdot \boldsymbol{n})(\boldsymbol{\tau}) \mathrm{d} \boldsymbol{\tau}\right| \leq c\left(\left\|\mathcal{A}_{K}^{-1} \boldsymbol{v}\right\|_{H^{s}(\hat{K})^{d}}+\left\|\operatorname{div}\left(\mathcal{A}_{K}^{-1} \boldsymbol{v}\right)\right\|_{L^{2}(\hat{K})}\right) .
$$


Standard arguments relying on (3.14), (3.15) and the use of intrinsic norm and seminorm on $H^{s}(\hat{K})$, see for instance [3], Section 7.43, give

$$
\left|\int_{e}(\boldsymbol{v} \cdot \boldsymbol{n})(\boldsymbol{\tau}) \mathrm{d} \boldsymbol{\tau}\right| \leq c\left(h_{K}^{\frac{d}{2}-1}\|\boldsymbol{v}\|_{L^{2}(K)^{d}}+h_{K}^{\frac{d}{2}+s-1}\|\boldsymbol{v}\|_{H^{s}(K)^{d}}+h_{K}^{\frac{d}{2}}\|\operatorname{div} \boldsymbol{v}\|_{L^{2}(K)}\right) .
$$

Inserting (3.21) and (3.22) into (3.20) leads to (3.19).

We now briefly prove analogous results for the operator $\Pi_{h}^{B R}$.

Lemma 3.6. The following property holds for any real number $s_{0}, 0 \leq s_{0} \leq 1$, for any $K$ in $\mathcal{T}_{h}^{F}$ and any $\boldsymbol{v}$ in $H^{s}\left(\Omega_{F}\right)^{d}, \frac{d}{2}<s \leq 2$,

$$
\left\|\boldsymbol{v}-\Pi_{h}^{B R} \boldsymbol{v}\right\|_{H^{s_{0}(K)^{d}}} \leq c h_{K}^{s-s_{0}}\|\boldsymbol{v}\|_{H^{s}(K)^{d}} .
$$

Proof. Let $\mathcal{I}_{h}$ denote the Lagrange interpolation operator with values in piecewise affine functions. It follows from the definition of $\Pi_{h}^{B R}$ that, if $\overline{\mathcal{E}}_{K}$ denotes the set of edges $(d=2)$ or faces $(d=3)$ of $K$,

$$
\left(\Pi_{h}^{B R} \boldsymbol{v}\right)_{\mid K}=\left(\mathcal{I}_{h} \boldsymbol{v}\right)_{\mid K}+\sum_{e \in \overline{\mathcal{E}}_{K}} \frac{\int_{e}\left(\left(\boldsymbol{v}-\mathcal{I}_{h} \boldsymbol{v}\right) \cdot \boldsymbol{n}_{e}\right)(\boldsymbol{\tau}) \mathrm{d} \boldsymbol{\tau}}{\int_{e} \psi_{e}(\boldsymbol{\tau}) \mathrm{d} \boldsymbol{\tau}} \psi_{e} \boldsymbol{n}_{e} .
$$

We recall the usual estimate, for $0 \leq r_{0} \leq 1$ and $\frac{d}{2}<r \leq 2$,

$$
\left\|\boldsymbol{v}-\mathcal{I}_{h} \boldsymbol{v}\right\|_{H^{r_{0}(K)^{d}}} \leq c h^{r-r_{0}}\|\boldsymbol{v}\|_{H^{r}(K)^{d}} .
$$

Applying this estimate with $r_{0}=s_{0}$ yields

$$
\left\|\boldsymbol{v}-\mathcal{I}_{h} \boldsymbol{v}\right\|_{H^{s_{0}}(K)^{d}} \leq c h_{K}^{s-s_{0}}\|\boldsymbol{v}\|_{H^{s}(K)^{d}} .
$$

On the other hand, we derive from standard arguments that

$$
\left\|\psi_{e} \boldsymbol{n}_{e}\right\|_{H^{s_{0}(K)^{d}}} \leq c h_{K}^{\frac{d}{2}-s_{0}}, \quad\left|\int_{e} \psi_{e}(\boldsymbol{\tau}) \mathrm{d} \boldsymbol{\tau}\right| \geq c^{\prime} h_{K}^{d-1} .
$$

Combining this with (3.22) and three applications of (3.24) gives for each $e$ in $\overline{\mathcal{E}}_{K}$

$$
\left|\frac{\int_{e}\left(\left(\boldsymbol{v}-\mathcal{I}_{h} \boldsymbol{v}\right) \cdot \boldsymbol{n}_{e}\right)(\boldsymbol{\tau}) \mathrm{d} \boldsymbol{\tau}}{\int_{e} \psi_{e}(\boldsymbol{\tau}) \mathrm{d} \boldsymbol{\tau}}\right|\left\|\psi_{e} \boldsymbol{n}_{e}\right\|_{H^{s_{0}}(K)^{d}} \leq c h_{K}^{s-s_{0}}\|\boldsymbol{v}\|_{H^{s}(K)^{d}} .
$$

This inequality and (3.25) yield the desired estimate.

To go further, we need the following result which is a consequence of Assumption 3.2.

Lemma 3.7. For each $h$, let $\lambda_{h}$ denote the maximal ratio $h_{K} / h_{K^{\prime}}$, where $K$ runs through $\mathcal{T}_{h}^{F}$, $K^{\prime}$ runs through $\mathcal{T}_{h}^{P}$ and $\partial K \cap \partial K^{\prime}$ has a positive $(d-1)$-measure. Then, all $\lambda_{h}$ are smaller than a constant $\lambda$ independent of $h$.

Proof. Let $K$ be any element of $\mathcal{T}_{h}^{F}$ which has an edge $(d=2)$ or a face $(d=3) e$ contained in $\Gamma$. Assumption 3.2 yields that $e$ is contained in the union of edges or faces $e_{i}, 1 \leq i \leq I$, of elements $K_{i}$ of $\mathcal{T}_{h}^{P}$, where $I$ is bounded independently of $K$ and $h$. So, we have

$$
\operatorname{meas}(e) \leq \sum_{i=1}^{I} \operatorname{meas}\left(e_{i}\right)
$$


On the other hand,

- $\operatorname{meas}(e)$ is equivalent to $h_{K}^{d-1}$ and each meas $\left(e_{i}\right)$ is equivalent to $h_{K_{i}}^{d-1}$, with equivalence constants only depending on the regularity parameter $\sigma$;

- when $e_{i}$ and $e_{j}$ are adjacent, i.e. share a vertex in dimension $d=2$ or an edge in dimension $d=3$, the ratio $h_{K_{i}} / h_{K_{j}}$ is bounded by constants only depending on $\sigma$;

- for all $e_{i}$ and $e_{j}$, there exists a path linking $e_{i}$ to $e_{j}$, only going from an $e$ to an adjacent $e^{\prime}$ and crossing at most $c$ elements $e$, where $c$ is bounded as a function of $I$.

Combining all this yields the desired result.

Lemma 3.8. If the data $(k, \boldsymbol{g})$ belong to $H^{\sigma_{P}}\left(\Gamma_{P}\right) \times H^{\sigma_{F}}\left(\Gamma_{F}\right)^{d}, \sigma_{P}>-\frac{1}{2}$ and $\sigma_{F}>\frac{d-1}{2}$, there exists a function $\boldsymbol{u}_{b h}$ in $\mathbb{X}_{h}$ which satisfies

$$
\boldsymbol{u}_{b h} \cdot \boldsymbol{n}=k_{h} \quad \text { on } \Gamma_{P} \quad \text { and } \quad \boldsymbol{u}_{b h}=\boldsymbol{g}_{h} \quad \text { on } \Gamma_{F},
$$

and

$$
\left\|\boldsymbol{u}_{b h}\right\|_{X(\Omega)} \leq c\left(\|k\|_{H^{\sigma_{P}\left(\Gamma_{P}\right)}}+\|\boldsymbol{g}\|_{\left.H^{\sigma_{F}\left(\Gamma_{F}\right)^{d}}\right)} .\right.
$$

Proof. We use once more the function $\boldsymbol{u}_{b}$ exhibited in Lemma 2.3 and, since it is constructed from the solutions of problems (2.12) and (2.14), we observe from [26], Section 7.3.3, or [19], Corollary 7, that, since $\Omega_{P}$ and $\Omega_{F}$ are polygons or polyhedra, there exist real numbers $s_{P}, 0<s_{P}<\sigma_{P}+\frac{1}{2}$, and $s_{F}, \frac{d}{2}<s_{F}<\sigma_{F}+\frac{1}{2}$, such that the pair $\left(\boldsymbol{u}_{b \mid \Omega_{P}}, \boldsymbol{u}_{b \mid \Omega_{F}}\right)$ belongs to $H^{s_{P}}\left(\Omega_{P}\right)^{d} \times H^{s_{F}}\left(\Omega_{F}\right)^{d}$ and satisfies

$$
\left\|\boldsymbol{u}_{b}\right\|_{H^{s} P\left(\Omega_{P}\right)^{d}}+\left\|\boldsymbol{u}_{b}\right\|_{H^{s} F\left(\Omega_{F}\right)^{d}} \leq c\left(\|k\|_{H^{\sigma_{P}\left(\Gamma_{P}\right)}}+\|\boldsymbol{g}\|_{H^{\sigma_{F}\left(\Gamma_{F}\right)^{d}}}\right) .
$$

The construction of the function $\boldsymbol{u}_{b h}$ is now performed in two steps.

1) We first introduce the function $\boldsymbol{w}_{h}^{1}$ such that

$$
\boldsymbol{w}_{h \mid \Omega_{P}}^{1}=\Pi_{h}^{R T} \boldsymbol{u}_{b \mid \Omega_{P}}, \quad \boldsymbol{w}_{h \mid \Omega_{F}}^{1}=\Pi_{h}^{B R} \boldsymbol{u}_{b \mid \Omega_{F}} .
$$

It follows from Lemmas 3.5 and 3.6 that, since $\boldsymbol{u}_{b}$ is divergence-free on $\Omega_{P}$,

$$
\left\|\boldsymbol{w}_{h}^{1}\right\|_{H\left(\operatorname{div}, \Omega_{P}\right)}+\left\|\boldsymbol{w}_{h}^{1}\right\|_{H^{1}\left(\Omega_{F}\right)^{d}} \leq c\left(\left\|\boldsymbol{u}_{b}\right\|_{H^{s} P\left(\Omega_{P}\right)^{d}}+\left\|\boldsymbol{u}_{b}\right\|_{H^{s} F\left(\Omega_{F}\right)^{d}}\right) .
$$

Moreover, owing to the definitions of $\Pi_{h}^{R T}$ and $\Pi_{h}^{B R}$, the function $\boldsymbol{w}_{h}^{1}$ satisfies the boundary conditions (3.26). 2) Recalling that $\mathcal{E}_{h}^{P, \Gamma}$ denotes the set of edges $(d=2)$ or faces $(d=3)$ of elements of $\mathcal{T}_{h}^{P}$ which are contained in $\bar{\Gamma}$, we consider the function $\boldsymbol{w}_{h}^{2}$ defined by

$$
\boldsymbol{w}_{h \mid \Omega_{P}}^{2}=\sum_{e \in \mathcal{E}_{h}^{P, \Gamma}}\left(\int_{e}\left(\left(\boldsymbol{w}_{h \mid \Omega_{F}}^{1}-\boldsymbol{w}_{h \mid \Omega_{P}}^{1}\right) \cdot \boldsymbol{n}\right)(\boldsymbol{\tau}) \mathrm{d} \boldsymbol{\tau}\right) \boldsymbol{\varphi}_{e}, \quad \boldsymbol{w}_{h \mid \Omega_{F}}^{2}=\mathbf{0}
$$

where the functions $\boldsymbol{\varphi}_{e}$ are defined in (3.17). We observe from the choice of $\boldsymbol{w}_{h}^{2}$ that the function $\boldsymbol{u}_{b h}=\boldsymbol{w}_{h}^{1}+\boldsymbol{w}_{h}^{2}$ satisfies the matching conditions (3.7), hence belongs to $\mathbb{X}_{h}$. Owing to the properties of the functions $\boldsymbol{\varphi}_{e}, \boldsymbol{w}_{h}^{2} \cdot \boldsymbol{n}$ vanishes on $\Gamma_{P}$, so that $\boldsymbol{u}_{b h}$ satisfies (3.26). Moreover, it follows from (3.15) and (3.21) that, if $K$ denotes the triangle of $\mathcal{T}_{h}^{P}$ that contains $e$,

$$
\left\|\varphi_{e}\right\|_{H(\operatorname{div}, K)} \leq c h_{K}^{-\frac{d}{2}} .
$$

Next, owing to the definition of $\boldsymbol{w}_{h}^{1}$, we have

$$
\int_{e}\left(\left(\boldsymbol{w}_{h \mid \Omega_{F}}^{1}-\boldsymbol{w}_{h \mid \Omega_{P}}^{1}\right) \cdot \boldsymbol{n}\right)(\boldsymbol{\tau}) \mathrm{d} \boldsymbol{\tau}=-\int_{e}\left(\left(\boldsymbol{u}_{b}-\boldsymbol{w}_{h \mid \Omega_{F}}^{1}\right) \cdot \boldsymbol{n}\right)(\boldsymbol{\tau}) \mathrm{d} \boldsymbol{\tau} .
$$


Applying (3.22) yields

$$
\begin{aligned}
\left|\int_{e}\left(\left(\boldsymbol{u}_{b}-\boldsymbol{w}_{h \mid \Omega_{F}}^{1}\right) \cdot \boldsymbol{n}\right)(\boldsymbol{\tau}) \mathrm{d} \boldsymbol{\tau}\right| \leq c \sum_{\kappa}\left(h_{\kappa}^{\frac{d}{2}-1}\left\|\boldsymbol{u}_{b}-\Pi_{h}^{B R} \boldsymbol{u}_{b}\right\|_{L^{2}(\kappa)^{d}}+h_{\kappa}^{\frac{d}{2}+s-1} \| \boldsymbol{u}_{b}-\right. & \Pi_{h}^{B R} \boldsymbol{u}_{b} \|_{H^{s}(\kappa)^{d}} \\
& \left.+h_{\kappa}^{\frac{d}{2}}\left\|\boldsymbol{u}_{b}-\Pi_{h}^{B R} \boldsymbol{u}_{b}\right\|_{H^{1}(\kappa)^{d}}\right),
\end{aligned}
$$

where the previous summation is taken on all the $\kappa$ in $\mathcal{T}_{h}^{F}$ such that $e \cap \partial \kappa$ has a positive measure. We use Lemma 3.6 to bound the norms on the $\kappa$. Combining all this with (3.30) yields

$$
\left|\int_{e}\left(\left(\boldsymbol{w}_{h \mid \Omega_{F}}^{1}-\boldsymbol{w}_{h \mid \Omega_{P}}^{1}\right) \cdot \boldsymbol{n}\right)(\boldsymbol{\tau}) \mathrm{d} \boldsymbol{\tau}\right|\left\|\boldsymbol{\varphi}_{e}\right\|_{H(\mathrm{div}, K)} \leq c h_{K}^{-\frac{d}{2}} \sum_{\kappa} h_{\kappa}^{\frac{d}{2}+s_{F}-1}\left\|\boldsymbol{u}_{b}\right\|_{H^{s_{F}(\kappa)^{d}}}
$$

Note also that the ratio $h_{\kappa}^{\frac{d}{2}} / h_{K}^{\frac{d}{2}}$ is bounded by $\lambda_{h}^{\frac{d}{2}}$, hence by a constant independent of $h$, see Lemma 3.7. This gives

$$
\left\|\boldsymbol{w}_{h}^{2}\right\|_{H\left(\operatorname{div}, \Omega_{P}\right)} \leq c h_{F}^{s_{F}-1}\left\|\boldsymbol{u}_{b}\right\|_{H^{s_{F}}\left(\Omega_{F}\right)^{d}} .
$$

Finally, estimate (3.27) is derived from (3.28), (3.29) and (3.31).

We prove a further result which is needed in Section 4. It requires the following parameters.

Notation 3.9. The parameters $\lambda_{P}$ and $\lambda_{F}$ are defined as follows:

(i) $\lambda_{P}$ is positive in the general case, equal to $1 / 4$ if $\Omega_{P}$ is a polygon $(d=2)$, equal to $1 / 2$ if $\Gamma_{a P}$ is empty or if there exists a convex neighbourhood in $\Omega_{P}$ of $\left(\bar{\Gamma}_{P} \cup \bar{\Gamma}\right) \cap \Gamma_{a P}$ and $<1$ if $\Gamma_{a P}$ is empty and $\Omega_{P}$ is a convex polygon or polyhedron;

(ii) $\lambda_{F}$ is equal to $1 / 2$ in the general case and to 1 if $\Omega_{F}$ is convex.

Corollary 3.10. If the assumptions of Lemma 3.8 are satisfied, the following estimates hold between the function $\boldsymbol{u}_{b}$ introduced in Lemma 2.3 and the function $\boldsymbol{u}_{b h}$ introduced in Lemma 3.8

$$
\left\|\boldsymbol{u}_{b}-\boldsymbol{u}_{b h}\right\|_{X(\Omega)} \leq c\left(h_{P}^{\min \left\{\sigma_{P}+\frac{1}{2}, \lambda_{P}\right\}}+h_{F}^{\min \left\{\sigma_{F}-\frac{1}{2}, \lambda_{F}\right\}}\right)\left(\|k\|_{H^{\sigma_{P}\left(\Gamma_{P}\right)}}+\|\boldsymbol{g}\|_{H^{\sigma_{F}\left(\Gamma_{F}\right)^{d}}}\right),
$$

and

$$
\sup _{q_{h} \in \mathbb{M}_{h}} \frac{\tilde{b}\left(\boldsymbol{u}_{b h}, q_{h}\right)}{\left\|q_{h}\right\|_{L^{2}(\Omega)}} \leq c h_{F}^{\min \left\{\sigma_{F}-\frac{1}{2}, \lambda_{F}\right\}}\left(\|k\|_{H^{\sigma_{P}\left(\Gamma_{P}\right)}}+\|\boldsymbol{g}\|_{H^{\sigma_{F}\left(\Gamma_{F}\right)^{d}}}\right) .
$$

Proof. Owing to the regularity properties of problems (2.12) and (2.14), see [26], Section 7.3.3, or [19], Corollary 3.7, estimate (3.28) holds with

$$
s_{P}=\min \left\{\sigma_{P}+\frac{1}{2}, \lambda_{P}\right\} \quad \text { and } \quad s_{F}=\min \left\{\sigma_{F}+\frac{1}{2}, \lambda_{F}+1\right\} .
$$

With the notation of the previous proof, since both $\boldsymbol{u}_{b}$ and $\Pi_{h}^{R T} \boldsymbol{u}_{b}$ are divergence-free on $\Omega_{P}$, we have the inequality

$$
\left\|\boldsymbol{u}_{b}-\boldsymbol{u}_{b h}\right\|_{X(\Omega)} \leq\left\|\boldsymbol{u}_{b}-\Pi_{h}^{R T} \boldsymbol{u}_{b}\right\|_{L^{2}\left(\Omega_{P}\right)^{d}}+\left\|\boldsymbol{u}_{b}-\Pi_{h}^{B R} \boldsymbol{u}_{b}\right\|_{H^{1}\left(\Omega_{F}\right)^{d}}+\left\|\boldsymbol{w}_{h}^{2}\right\|_{H\left(\operatorname{div}, \Omega_{P}\right)} .
$$

The approximation properties of the operator $\Pi_{h}^{R T}$ are easily derived from the fact that it preserves the constants on each $K$ in $\mathcal{T}_{h}^{P}$, by applying (3.19) to the function $\boldsymbol{v}-\mathbf{c}_{K}$ for an appropriate constant $\mathbf{c}_{K}$ and using the approximation properties of this constant. They read, for $0<r \leq 1$,

$$
\left\|\boldsymbol{v}-\Pi_{h}^{R T} \boldsymbol{v}\right\|_{L^{2}(K)^{d}} \leq c h^{r}\|\boldsymbol{v}\|_{H^{r}(K)^{d}}
$$


So, using (3.35) to bound the first term in the right-hand side of (3.34), (3.23) to bound the second term and (3.31) to bound the third term yields (3.32). We also derive from the properties (3.12) and (3.13) of the operators $\Pi_{h}^{R T}$ and $\Pi_{h}^{B R}$ that, since $\boldsymbol{u}_{b}$ is divergence-free on $\Omega$, we have for all $q_{h}$ in $\mathbb{M}_{h}$,

$$
\tilde{b}\left(\boldsymbol{w}_{h}^{1}, q_{h}\right)=\sum_{K \in \mathcal{T}_{h}^{P} \cup \mathcal{T}_{h}^{F}} q_{h \mid K} \int_{\partial K}\left(\boldsymbol{w}_{h}^{1} \cdot \boldsymbol{n}\right)(\boldsymbol{\tau}) \mathrm{d} \boldsymbol{\tau}=\sum_{K \in \mathcal{T}_{h}^{P} \cup \mathcal{T}_{h}^{F}} q_{h \mid K} \int_{\partial K}\left(\boldsymbol{u}_{b} \cdot \boldsymbol{n}\right)(\boldsymbol{\tau}) \mathrm{d} \boldsymbol{\tau}=0,
$$

so that

and we derive (3.33) from (3.31).

$$
\tilde{b}\left(\boldsymbol{u}_{b h}, q_{h}\right)=\tilde{b}\left(\boldsymbol{w}_{h}^{2}, q_{h}\right) \leq\left\|\boldsymbol{w}_{h}^{2}\right\|_{H\left(\operatorname{div}, \Omega_{P}\right)}\left\|q_{h}\right\|_{L^{2}(\Omega)},
$$

In analogy with Section 2, we now set: $\boldsymbol{u}_{0 h}=\boldsymbol{u}_{h}-\boldsymbol{u}_{b h}$, where $\boldsymbol{u}_{b h}$ is the function exhibited in Lemma 3.8. This leads to consider the problem:

Find $\left(\boldsymbol{u}_{0 h}, p_{h}\right)$ in $\mathbb{X}_{0 h} \times \mathbb{M}_{h}$ such that

$$
\begin{aligned}
& \forall \boldsymbol{v}_{h} \in \mathbb{X}_{0 h}, \quad a\left(\boldsymbol{u}_{0 h}, \boldsymbol{v}_{h}\right)+\tilde{b}\left(\boldsymbol{v}_{h}, p_{h}\right)=-a\left(\boldsymbol{u}_{b h}, \boldsymbol{v}_{h}\right)+\mathcal{L}\left(\boldsymbol{v}_{h}\right), \\
& \forall q_{h} \in \mathbb{M}_{h}, \quad \tilde{b}\left(\boldsymbol{u}_{0 h}, q_{h}\right)=-\tilde{b}\left(\boldsymbol{u}_{b h}, q_{h}\right) .
\end{aligned}
$$

We also introduce the discrete kernel

$$
\mathbb{V}_{h}=\left\{\boldsymbol{v}_{h} \in \mathbb{X}_{0 h} ; \forall q \in \mathbb{M}_{h}, \tilde{b}\left(\boldsymbol{v}_{h}, q_{h}\right)=0\right\} .
$$

It must be noted that the functions in $\mathbb{V}_{h}$ are divergence-free only on $\Omega_{P}$. We now study the properties of the forms $a(\cdot, \cdot)$ and $\tilde{b}(\cdot, \cdot)$ on the discrete spaces.

Lemma 3.11. If $\Gamma_{F}$ has a positive measure in $\partial \Omega_{F}$, there exists a constant $\tilde{\alpha}>0$ such that the following ellipticity property holds

$$
\forall \boldsymbol{v}_{h} \in \mathbb{V}_{h}, \quad a\left(\boldsymbol{v}_{h}, \boldsymbol{v}_{h}\right) \geq \tilde{\alpha}\left\|\boldsymbol{v}_{h}\right\|_{X(\Omega)}^{2} .
$$

Proof. Since functions in $\mathbb{V}_{h}$ are divergence-free on $\Omega_{P}$, properties (2.21) and (2.22) still hold for all functions $\boldsymbol{v}_{h}$ in $\mathbb{V}_{h}$. So, we now wish to check that

$$
\forall \boldsymbol{v}_{h} \in \mathbb{V}_{h}, \quad\left\|\boldsymbol{v}_{h}\right\|_{L^{2}\left(\Omega_{F}\right)^{d}} \leq c\left|\boldsymbol{v}_{h}\right|_{H^{1}\left(\Omega_{F}\right)^{d}} .
$$

When $\Gamma_{F}$ has a positive measure, this inequality is a simple consequence of the Poincaré-Friedrichs inequality and of the imbedding of $\mathbb{X}_{0 h}^{F}$ into the space of functions in $H^{1}\left(\Omega_{F}\right)$ vanishing on $\Gamma_{F}$.

Remark 3.12. When $\Gamma_{F}$ has a zero measure but the normal vector $\boldsymbol{n}(\boldsymbol{x})$ when $\boldsymbol{x}$ runs through $\Gamma$ runs through a basis of $\mathbb{R}^{d}$ (this is the second possible assumption of Lem. 2.5), it is readily checked that any element of $\mathbb{V}_{h}$ such that $a\left(\boldsymbol{v}_{h}, \boldsymbol{v}_{h}\right)=0$ is equal to zero. Thus, using the equivalence of norms on the finite-dimensional space $\mathbb{V}_{h}$ yields that there exists a constant $\alpha_{h}$ positive but depending on the triangulations $\mathcal{T}_{h}^{P}$ and $\mathcal{T}_{h}^{F}$ such that

$$
\forall \boldsymbol{v}_{h} \in \mathbb{V}_{h}, \quad a\left(\boldsymbol{v}_{h}, \boldsymbol{v}_{h}\right) \geq \alpha_{h}\left\|\boldsymbol{v}_{h}\right\|_{X(\Omega)}^{2} .
$$

However the standard arguments to evaluate the dependence of $\alpha_{h}$ with respect to $h_{P}$ and $h_{F}$ seem to fail here. Fortunately, the assumption that $\Gamma_{F}$ has a positive measure in $\partial \Omega_{F}$ is not restrictive for the applications that we wish to consider.

We now prove the inf-sup condition on $\tilde{b}(\cdot, \cdot)$. It requires the modified Bernardi-Raugel operator $\widetilde{\Pi}_{h}^{B R}$ defined as follows: if $\mathcal{R}_{h}$ denotes a Clément type regularization operator with values in the space of piecewise affine functions which vanish on $\Gamma_{F}$ (see for instance [9], Sect. IX.3, for a detailed definition of such an operator),

$$
\left(\widetilde{\Pi}_{h}^{B R} \boldsymbol{v}\right)_{\mid K}=\left(\mathcal{R}_{h} \boldsymbol{v}\right)_{\mid K}+\sum_{e \in \overline{\mathcal{E}}_{K}} \frac{\int_{e}\left(\left(\boldsymbol{v}-\mathcal{R}_{h} \boldsymbol{v}\right) \cdot \boldsymbol{n}_{e}\right)(\boldsymbol{\tau}) \mathrm{d} \boldsymbol{\tau}}{\int_{e} \psi_{e}(\boldsymbol{\tau}) \mathrm{d} \boldsymbol{\tau}} \psi_{e} \boldsymbol{n}_{e} .
$$


Lemma 3.13. There exist two constants $h_{0}>0$ and $\tilde{\beta}>0$ such that, either when both $\Gamma_{a P}$ and $\Gamma_{a F}$ have a positive measure or for all $h \leq h_{0}$, the following inf-sup condition holds

$$
\forall q_{h} \in \mathbb{M}_{h}, \quad \sup _{\boldsymbol{v}_{h} \in \mathbb{X}_{0 h}} \frac{\tilde{b}\left(\boldsymbol{v}_{h}, q_{h}\right)}{\left\|\boldsymbol{v}_{h}\right\|_{X(\Omega)}} \geq \tilde{\beta}\left\|q_{h}\right\|_{L^{2}(\Omega)} .
$$

We must prove this lemma in the next three situations: When both $\Gamma_{a P}$ and $\Gamma_{a F}$ have a positive measure, when $\Gamma_{a P}$ has a zero measure and when $\Gamma_{a F}$ has a zero measure. However, we skip the proof in the third situation since it is less realistic than the second one (see Fig. 1) and the arguments are exactly the same.

Proof. Case where $\Gamma_{a P}$ and $\Gamma_{a F}$ have a positive measure.

In this situation, it follows from exactly the same arguments as in the proof of Lemma 2.6 that, for any function $q_{h}$ in $\mathbb{M}_{h}$, there exists a function $\boldsymbol{v}_{P}$ in $H^{1}\left(\Omega_{P}\right)^{d}$, vanishing on $\Gamma_{P}$ and also on $\Gamma$ such that

$$
\operatorname{div} \boldsymbol{v}_{P}=-q_{h} \quad \text { on } \Omega_{P} \quad \text { and } \quad\left\|\boldsymbol{v}_{P}\right\|_{H^{1}\left(\Omega_{P}\right)^{d}} \leq c\left\|q_{h}\right\|_{L^{2}\left(\Omega_{P}\right)},
$$

and also a function $\boldsymbol{v}_{F}$ in $H^{1}\left(\Omega_{F}\right)^{d}$, vanishing on $\Gamma_{F} \cup \Gamma$ such that

$$
\operatorname{div} \boldsymbol{v}_{F}=-q_{h} \quad \text { on } \Omega_{F} \quad \text { and } \quad\left\|\boldsymbol{v}_{F}\right\|_{H^{1}\left(\Omega_{F}\right)^{d}} \leq c\left\|q_{h}\right\|_{L^{2}\left(\Omega_{F}\right)} .
$$

We now define

$$
\boldsymbol{v}_{h \mid \Omega_{P}}=\Pi_{h}^{R T} \boldsymbol{v}_{P}, \quad \boldsymbol{v}_{h \mid \Omega_{F}}=\widetilde{\Pi}_{h}^{B R} \boldsymbol{v}_{F} .
$$

Only for this proof, we make the further assumption that the operator $\mathcal{R}_{h}$ takes its values in the space of piecewise affine functions which also vanish on $\Gamma$, so that $\widetilde{\Pi}_{h}^{B R} \boldsymbol{v}_{F}$ vanishes on $\Gamma_{F} \cup \Gamma$. On the other hand, it is readily checked that all functions $\boldsymbol{v}_{K}$ in $\mathcal{P}_{R T}(K)$ are such that $\boldsymbol{v}_{K} \cdot \boldsymbol{n}$ is constant on each edge $(d=2)$ or face $(d=3)$ of $K$, so that $\Pi_{h}^{R T} \boldsymbol{v}_{P} \cdot \boldsymbol{n}$ vanishes on $\Gamma_{P} \cup \Gamma$. These two properties yield that the function $\boldsymbol{v}_{h}$ satisfies that matching conditions (3.7), hence belongs to $\mathbb{X}_{0 h}$. We also have

$$
\tilde{b}\left(\boldsymbol{v}_{h}, q_{h}\right)=-\left.\sum_{K \in \mathcal{T}_{h}^{P}} q_{h}\right|_{K} \int_{\partial K}\left(\boldsymbol{v}_{h} \cdot \boldsymbol{n}\right)(\boldsymbol{\tau}) \mathrm{d} \boldsymbol{\tau}-\left.\sum_{K \in \mathcal{T}_{h}^{F}} q_{h}\right|_{K} \int_{\partial K}\left(\boldsymbol{v}_{h} \cdot \boldsymbol{n}\right)(\boldsymbol{\tau}) \mathrm{d} \boldsymbol{\tau} .
$$

So it follows from the definition of the operators $\Pi_{h}^{R T}$ and $\widetilde{\Pi}_{h}^{B R}$ that

$$
\begin{aligned}
\tilde{b}\left(\boldsymbol{v}_{h}, q_{h}\right) & =-\left.\sum_{K \in \mathcal{T}_{h}^{P}} q_{h}\right|_{K} \int_{\partial K}\left(\boldsymbol{v}_{P} \cdot \boldsymbol{n}\right)(\boldsymbol{\tau}) \mathrm{d} \boldsymbol{\tau}-\left.\sum_{K \in \mathcal{T}_{h}^{F}} q_{h}\right|_{K} \int_{\partial K}\left(\boldsymbol{v}_{F} \cdot \boldsymbol{n}\right)(\boldsymbol{\tau}) \mathrm{d} \boldsymbol{\tau} \\
& =-\int_{\Omega_{P}}\left(\operatorname{div} \boldsymbol{v}_{P}\right)(\boldsymbol{x}) q_{h}(\boldsymbol{x}) \mathrm{d} \boldsymbol{x}-\int_{\Omega_{F}}\left(\operatorname{div} \boldsymbol{v}_{F}\right)(\boldsymbol{x}) q_{h}(\boldsymbol{x}) \mathrm{d} \boldsymbol{x} .
\end{aligned}
$$

Combining this with (3.42) and (3.43) yields

$$
\tilde{b}\left(\boldsymbol{v}_{h}, q_{h}\right)=\left\|q_{h}\right\|_{L^{2}(\Omega)}^{2} .
$$

We also deduce from Lemma 3.5 that

$$
\left\|\boldsymbol{v}_{h}\right\|_{H\left(\mathrm{div}, \Omega_{P}\right)} \leq c\left\|\boldsymbol{v}_{P}\right\|_{H^{1}\left(\Omega_{P}\right)^{d}}
$$

whence, from (3.42),

$$
\left\|\boldsymbol{v}_{h}\right\|_{H\left(\mathrm{div}, \Omega_{P}\right)} \leq c\left\|q_{h}\right\|_{L^{2}\left(\Omega_{P}\right)} .
$$


The same arguments as in the proof of Lemma 3.6, with (3.24) replaced by (see [9], Chap. IX, Th. 3.11)

$$
\left\|\boldsymbol{v}-\mathcal{R}_{h} \boldsymbol{v}\right\|_{H^{s_{0}}(K)^{d}} \leq c h_{K}^{1-s_{0}}\|\boldsymbol{v}\|_{H^{1}\left(\Delta_{K}\right)^{d}},
$$

where $\Delta_{K}$ is the union of elements $\kappa$ of $\mathcal{T}_{h}^{F}$ such that $K \cap \kappa$ is not empty, lead to

$$
\left\|\boldsymbol{v}_{h}\right\|_{H^{1}\left(\Omega_{F}\right)^{d}} \leq c\left\|\boldsymbol{v}_{F}\right\|_{H^{1}\left(\Omega_{F}\right)^{d}},
$$

whence, owing to (3.43),

$$
\left\|\boldsymbol{v}_{h}\right\|_{H^{1}\left(\Omega_{F}\right)^{d}} \leq c\left\|q_{h}\right\|_{L^{2}\left(\Omega_{F}\right)} .
$$

The desired inf-sup condition now follows from (3.44), (3.45) and (3.46).

Proof. Case where $\Gamma_{a P}$ has a zero measure.

Let $\varphi_{\Gamma}$ be a smooth vector field with support contained in the interior of $\Gamma$ such that

$$
\int_{\Gamma}\left(\boldsymbol{\varphi}_{\Gamma} \cdot \boldsymbol{n}\right)(\boldsymbol{\tau}) \mathrm{d} \boldsymbol{\tau}=1
$$

We define $\varphi_{\Gamma h}$ in the following way: On $\Omega_{F}, \varphi_{\Gamma h}$ is affine on all elements $K$ of $\mathcal{T}_{h}^{F}$ and is equal to $\varphi_{\Gamma}(\boldsymbol{a})$ at all vertices $\boldsymbol{a}$ of these elements that belong to $\Gamma$ and to zero at all other vertices; on $\Omega_{P}$, we set

$$
\boldsymbol{\varphi}_{\Gamma h \mid \Omega_{P}}=\sum_{e \in \mathcal{E}_{h}^{P, \Gamma}}\left(\int_{e}\left(\boldsymbol{\varphi}_{\Gamma h \mid \Omega_{F}} \cdot \boldsymbol{n}\right)(\boldsymbol{\tau}) \mathrm{d} \boldsymbol{\tau}\right) \boldsymbol{\varphi}_{e} .
$$

Thus, it is readily checked that $\varphi_{\Gamma h}$ belongs to $\mathbb{X}_{h}^{0}$ and moreover that, when $h$ is small enough,

$$
\int_{\Gamma}\left(\boldsymbol{\varphi}_{\Gamma h} \cdot \boldsymbol{n}\right)(\boldsymbol{\tau}) \mathrm{d} \boldsymbol{\tau} \geq \frac{1}{2}
$$

For a while, we set

$$
b_{P}(\boldsymbol{v}, q)=-\int_{\Omega_{P}}\left(\operatorname{div} \boldsymbol{v}_{\mid \Omega_{P}}\right)(\boldsymbol{x}) q(\boldsymbol{x}) \mathrm{d} \boldsymbol{x}, \quad b_{F}(\boldsymbol{v}, q)-\int_{\Omega_{F}}\left(\operatorname{div} \boldsymbol{v}_{\mid \Omega_{F}}\right)(\boldsymbol{x}) q(\boldsymbol{x}) \mathrm{d} \boldsymbol{x} .
$$

Next, we proceed in two steps.

1) On $\Omega_{P}$, we use the decomposition

$$
q_{h \mid \Omega_{P}}=\tilde{q}_{h}+\bar{q}_{h}, \quad \text { with } \quad \bar{q}_{h}=\frac{1}{\operatorname{meas}\left(\Omega_{P}\right)} \int_{\Omega_{P}} q_{h}(\boldsymbol{x}) \mathrm{d} \boldsymbol{x} .
$$

Indeed, there exists a stable function $\boldsymbol{v}$ in $H_{0}^{1}\left(\Omega_{P}\right)^{d}$ such that $-\operatorname{div} \boldsymbol{v}=\tilde{q}_{h}$; then, the function $\tilde{\boldsymbol{v}}_{h}=\Pi_{h}^{R T} \boldsymbol{v}$ belongs to $X_{h}^{P} \cap H_{0}\left(\operatorname{div}, \Omega_{P}\right)$ and satisfies

$$
b_{P}\left(\tilde{\boldsymbol{v}}_{h}, \tilde{q}_{h}\right)=\left\|\tilde{q}_{h}\right\|_{L^{2}\left(\Omega_{P}\right)}^{2} \quad \text { and } \quad\left\|\tilde{\boldsymbol{v}}_{h}\right\|_{H\left(\mathrm{div}, \Omega_{P}\right)} \leq c\left\|\tilde{q}_{h}\right\|_{L^{2}\left(\Omega_{P}\right)} .
$$

On the other hand, it is readily checked by integration by parts and also from (3.47) that the function

$$
\overline{\boldsymbol{v}}_{h}=-\bar{q}_{h} \frac{\operatorname{meas}\left(\Omega_{P}\right)}{\int_{\Gamma}\left(\boldsymbol{\varphi}_{\Gamma h} \cdot \boldsymbol{n}\right)(\boldsymbol{\tau}) \mathrm{d} \boldsymbol{\tau}} \boldsymbol{\varphi}_{\Gamma h}
$$

satisfies

$$
b_{P}\left(\overline{\boldsymbol{v}}_{h}, \bar{q}_{h}\right)=\left\|\bar{q}_{h}\right\|_{L^{2}\left(\Omega_{P}\right)}^{2} \quad \text { and } \quad\left\|\overline{\boldsymbol{v}}_{h}\right\|_{X(\Omega)} \leq c\left\|\bar{q}_{h}\right\|_{L^{2}\left(\Omega_{P}\right)}
$$


Thus, applying the Boland and Nicolaides argument, see [13], which relies on the orthogonality properties

$$
b_{P}\left(\tilde{\boldsymbol{v}}_{h}, \bar{q}_{h}\right)=0, \quad \int_{\Omega_{P}} \tilde{q}_{h}(\boldsymbol{x}) \bar{q}_{h}(\boldsymbol{x}) \mathrm{d} \boldsymbol{x}=0,
$$

gives the existence of a constant $\mu$ independent of $h$ such that the function $\boldsymbol{v}_{h \mid \Omega_{P}}=\tilde{\boldsymbol{v}}_{h}+\mu \overline{\boldsymbol{v}}_{h}$ satisfies

$$
b_{P}\left(\boldsymbol{v}_{h}, q_{h}\right) \geq c\left\|q_{h}\right\|_{L^{2}\left(\Omega_{P}\right)}^{2} \quad \text { and } \quad\left\|\boldsymbol{v}_{h}\right\|_{H\left(\operatorname{div}, \Omega_{P}\right)} \leq c^{\prime}\left\|q_{h}\right\|_{L^{2}\left(\Omega_{P}\right)} .
$$

2) It follows from the definition of $\boldsymbol{\varphi}_{\Gamma h}$ that $\left(\operatorname{div} \overline{\boldsymbol{v}}_{h}\right)_{\mid \Omega_{F}}$ is constant on each element of $\mathcal{T}_{h}^{F}$. Thus, Lemma 2.6 yields the existence of a function $\boldsymbol{v}$ in $H^{1}\left(\Omega_{F}\right)^{d}$, vanishing on $\Gamma_{F} \cup \Gamma$, such that $-\operatorname{div} \boldsymbol{v}$ is equal to $q_{h}+\operatorname{div}\left(\mu \overline{\boldsymbol{v}}_{h}\right)$ and applying the modified Bernardi-Raugel operator $\widetilde{\Pi}_{h}^{B R}$ defined in (3.40) to it yields that the function $\boldsymbol{v}_{h \mid \Omega_{F}}=$ $\widetilde{\Pi}_{h}^{B R} \boldsymbol{v}+\mu \overline{\boldsymbol{v}}_{h}$ satisfies

$$
b_{F}\left(\boldsymbol{v}_{h}, q_{h}\right)=\left\|q_{h}\right\|_{L^{2}\left(\Omega_{F}\right)}^{2} \quad \text { and } \quad\left\|\boldsymbol{v}_{h}\right\|_{H^{1}\left(\Omega_{F}\right)^{d}} \leq c\left(\left\|q_{h}\right\|_{L^{2}\left(\Omega_{F}\right)}+\left\|\overline{\boldsymbol{v}}_{h}\right\|_{X(\Omega)}\right) .
$$

To conclude, we observe that the function $\boldsymbol{v}_{h}$ belongs to $\mathbb{X}_{0 h}$. The desired inf-sup condition is then derived from (3.50), (3.51) and (3.49).

From now on, we assume that $h$ is small enough for the inf-sup condition (3.41) to hold. Indeed, this condition is only needed when $\Gamma_{a P}$ or $\Gamma_{a F}$ has a zero measure and, with the notation of the previous proof, can be written, when $\Gamma_{a P}$ has a zero measure for instance,

$$
\left\|\varphi_{\Gamma}-\varphi_{\Gamma h}\right\|_{L^{1}(\Gamma)} \leq \frac{1}{2}
$$

So, since $\varphi_{\Gamma}$ is very smooth, it is not at all restrictive. Owing to the previous lemmas, we are now in a position to prove the main result of this section.

Theorem 3.14. Assume that $\Gamma_{F}$ has a positive measure in $\partial \Omega_{F}$. Then, for any data $\left(k, \boldsymbol{g}, \boldsymbol{f}, p_{a}, \mathbf{t}_{a}\right)$ satisfying

$$
k \in H^{\sigma_{P}}\left(\Gamma_{P}\right), \quad \boldsymbol{g} \in H^{\sigma_{F}}\left(\Gamma_{F}\right)^{d}, \quad \boldsymbol{f} \in L^{2}(\Omega)^{d}, \quad p_{a} \in H_{00}^{\frac{1}{2}}\left(\Gamma_{a P}\right), \quad \mathbf{t}_{a} \in H^{-\frac{1}{2}}\left(\Gamma_{a F}\right)^{d},
$$

for some real numbers $\sigma_{P}>-\frac{1}{2}$ and $\sigma_{F}>\frac{d-1}{2}$, problem (3.9)-(3.10) has a unique solution $\left(\boldsymbol{u}_{h}, p_{h}\right)$ in $\mathbb{X}_{h} \times \mathbb{M}_{h}$. Moreover this solution satisfies

$$
\begin{aligned}
\left\|\boldsymbol{u}_{h}\right\|_{X(\Omega)}+\left\|p_{h}\right\|_{L^{2}(\Omega)} \leq c\left(\|k\|_{H^{\sigma_{P}\left(\Gamma_{P}\right)}}\right. & +\|\boldsymbol{g}\|_{H^{\sigma_{F}}\left(\Gamma_{F}\right)^{d}}+\|\boldsymbol{f}\|_{L^{2}(\Omega)^{d}} \\
& \left.+\left\|p_{a}\right\|_{H_{00}^{\frac{1}{2}\left(\Gamma_{a P}\right)}}+\left\|\mathbf{t}_{a}\right\|_{H^{-\frac{1}{2}\left(\Gamma_{a F}\right)^{d}}}\right) .
\end{aligned}
$$

Proof. We check separately the existence and the uniqueness.

1) Let $\boldsymbol{u}_{b h}$ denote the function exhibited in Lemma 3.8. It follows from the ellipticity property (3.38) and the inf-sup condition (3.41), see [24], Chapter I, Theorem 4.1, that problem (3.36) has a unique solution $\left(\boldsymbol{u}_{0 h}, p_{h}\right)$ in $\mathbb{X}_{0 h} \times \mathbb{M}_{h}$ which moreover satisfies

$$
\left\|\boldsymbol{u}_{0 h}\right\|_{X(\Omega)}+\left\|p_{h}\right\|_{L^{2}(\Omega)} \leq c\left(\left\|\boldsymbol{u}_{b h}\right\|_{X(\Omega)}+\|\boldsymbol{f}\|_{L^{2}(\Omega)^{d}}+\left\|p_{a}\right\|_{H_{00}^{\frac{1}{2}\left(\Gamma_{a P}\right)}}+\left\|\mathbf{t}_{a}\right\|_{H^{-\frac{1}{2}}\left(\Gamma_{a F}\right)^{d}}\right) .
$$

Then, the pair $\left(\boldsymbol{u}_{h}=\boldsymbol{u}_{0 h}+\boldsymbol{u}_{b h}, p_{h}\right)$ is a solution of problem (3.9)-(3.10), and estimate (3.53) is a direct consequence of (3.27) and (3.54).

2) If all data $\left(k, \boldsymbol{g}, \boldsymbol{f}, p_{a}, \mathbf{t}_{a}\right)$ are equal to zero, $\left(\boldsymbol{u}_{h}, p_{h}\right)$ is a solution of problem (3.36) with the right-hand sides of the two equations equal to zero. Thus, it follows from (3.38) and (3.41) that it is equal to zero. So, the solution of problem (3.9)-(3.10) is unique. 
Remark 3.15. The regularity assumptions that are made on the data $\boldsymbol{f}$ in Theorem 3.14 can easily be weakened: It suffices to enforce that $\boldsymbol{f}_{\mid \Omega_{P}}$ belongs to the dual space of functions on $H\left(\operatorname{div}, \Omega_{P}\right)$ with zero normal traces on $\Gamma_{P}$ and $\boldsymbol{f}_{\mid \Omega_{F}}$ belongs to the dual space of functions on $H^{1}\left(\Omega_{F}\right)^{d}$ vanishing on $\Gamma_{F}$. However we have no direct application for this weaker regularity.

\section{A PRIORI ERROR ESTIMATES}

We intend to prove an error estimate between the solution $(\boldsymbol{u}, p)$ of problem (2.6)-(2.7) and the solution $\left(\boldsymbol{u}_{h}, p_{h}\right)$ of problem (3.9)-(3.10). The main difficulty here is that applying the interpolation operator $\mathcal{I}_{h}$ or the operator $\Pi_{h}^{B R}$ to the solution $\boldsymbol{u}_{\mid \Omega_{F}}$ (in order to recover the boundary condition $\boldsymbol{g}_{h}$ of the discrete problem) would require that $\boldsymbol{u}_{\mid \Omega_{F}}$ is continuous on $\bar{\Omega}_{F}$. In view of Proposition 2.8, this assumption is not likely, at least in dimension $d=3$. So we prefer to follow another approach, based on the triangle inequality

$$
\left\|\boldsymbol{u}-\boldsymbol{u}_{h}\right\|_{X(\Omega)} \leq\left\|\boldsymbol{u}_{b}-\boldsymbol{u}_{b h}\right\|_{X(\Omega)}+\left\|\boldsymbol{u}_{0}-\boldsymbol{u}_{0 h}\right\|_{X(\Omega)},
$$

where the functions $\boldsymbol{u}_{b}$ and $\boldsymbol{u}_{b h}$ are introduced in Lemmas 2.3 and 3.8, respectively.

An estimate for the quantity $\left\|\boldsymbol{u}_{b}-\boldsymbol{u}_{b h}\right\|_{X(\Omega)}$ is established in Corollary 3.10. So we are now interested in proving the following version of the second Strang's lemma for problems (2.18) and (3.36), the main difficulty being due to the nonconformity of the mortar element discretization.

Lemma 4.1. Assume that $\Gamma_{F}$ has a positive measure in $\partial \Omega_{F}$. The following estimate holds between the solution $\left(\boldsymbol{u}_{0}, p\right)$ of problem (2.18) and the solution $\left(\boldsymbol{u}_{0 h}, p_{h}\right)$ of problem (3.36)

$$
\begin{aligned}
\left\|\boldsymbol{u}_{0}-\boldsymbol{u}_{0 h}\right\|_{X(\Omega)} \leq & c\left(\inf _{\boldsymbol{w}_{h} \in \mathbb{V}_{h}}\left\|\boldsymbol{u}_{0}-\boldsymbol{w}_{h}\right\|_{X(\Omega)}+\inf _{r_{h} \in \mathbb{M}_{h}}\left\|p-r_{h}\right\|_{L^{2}(\Omega)}\right. \\
& \left.+\left\|\boldsymbol{u}_{b}-\boldsymbol{u}_{b h}\right\|_{X(\Omega)}+\sup _{q_{h} \in \mathbb{M}_{h}} \frac{\tilde{b}\left(\boldsymbol{u}_{b h}, q_{h}\right)}{\left\|q_{h}\right\|_{L^{2}(\Omega)}}+\sup _{\boldsymbol{v}_{h} \in \mathbb{X}_{0 h}} \frac{\int_{\Gamma}\left(\left(\boldsymbol{v}_{h \mid \Omega_{P}}-\boldsymbol{v}_{h \mid \Omega_{F}}\right) \cdot \boldsymbol{n}\right)(\boldsymbol{\tau}) p_{\mid \Omega_{P}}(\boldsymbol{\tau}) \mathrm{d} \boldsymbol{\tau}}{\left\|\boldsymbol{v}_{h}\right\|_{X(\Omega)}}\right) .
\end{aligned}
$$

Proof. It is divided in three steps.

1) Owing to the inf-sup condition (3.41), there exists [24], Chapter I, Lemma 4.1, a function $\tilde{\boldsymbol{u}}_{h}$ in $\mathbb{X}_{0 h}$ such that

$$
\forall q_{h} \in \mathbb{M}_{h}, \quad \tilde{b}\left(\tilde{\boldsymbol{u}}_{h}, q_{h}\right)=\tilde{b}\left(\boldsymbol{u}_{0 h}, q_{h}\right),
$$

and, by using the second line of (3.36),

$$
\left\|\tilde{\boldsymbol{u}}_{h}\right\|_{X(\Omega)} \leq \tilde{\beta}^{-1} \sup _{q_{h} \in \mathbb{M}_{h}} \frac{\tilde{b}\left(\boldsymbol{u}_{b h}, q_{h}\right)}{\left\|q_{h}\right\|_{L^{2}(\Omega)}}
$$

Then, the function $\tilde{\boldsymbol{u}}_{0 h}=\boldsymbol{u}_{0 h}-\tilde{\boldsymbol{u}}_{h}$ belongs to $\mathbb{V}_{h}$ and satisfies

$$
\forall \boldsymbol{v}_{h} \in \mathbb{V}_{h}, \quad a\left(\tilde{\boldsymbol{u}}_{0 h}, \boldsymbol{v}_{h}\right)=-a\left(\boldsymbol{u}_{b h}, \boldsymbol{v}_{h}\right)-a\left(\tilde{\boldsymbol{u}}_{h}, \boldsymbol{v}_{h}\right)+\mathcal{L}\left(\boldsymbol{v}_{h}\right) .
$$

2) When multiplying the first lines of (1.1) and (1.2) by a function $\boldsymbol{v}_{h}$ of $\mathbb{V}_{h}$, integrating by parts and summing the two resulting equations, we obtain

$$
a\left(\boldsymbol{u}, \boldsymbol{v}_{h}\right)+\tilde{b}\left(\boldsymbol{v}_{h}, p\right)=\mathcal{L}\left(\boldsymbol{v}_{h}\right)-\int_{\Gamma}\left(\left(\boldsymbol{v}_{h \mid \Omega_{P}}-\boldsymbol{v}_{h \mid \Omega_{F}}\right) \cdot \boldsymbol{n}\right)(\boldsymbol{\tau}) p_{\mid \Omega_{P}}(\boldsymbol{\tau}) \mathrm{d} \boldsymbol{\tau} .
$$


This last equation can be written equivalently as

$$
\begin{aligned}
\forall \boldsymbol{v}_{h} \in \mathbb{V}_{h}, \quad a\left(\boldsymbol{u}_{0}, \boldsymbol{v}_{h}\right)+\tilde{b}\left(\boldsymbol{v}_{h}, p\right)= & -a\left(\boldsymbol{u}_{b}, \boldsymbol{v}_{h}\right)+\mathcal{L}\left(\boldsymbol{v}_{h}\right) \\
& -\int_{\Gamma}\left(\left(\boldsymbol{v}_{h \mid \Omega_{P}}-\boldsymbol{v}_{h \mid \Omega_{F}}\right) \cdot \boldsymbol{n}\right)(\boldsymbol{\tau}) p_{\mid \Omega_{P}}(\boldsymbol{\tau}) \mathrm{d} \boldsymbol{\tau} .
\end{aligned}
$$

3) Let now $\boldsymbol{w}_{h}$ and $r_{h}$ be any elements of $\mathbb{V}_{h}$ and $\mathbb{M}_{h}$, respectively. It follows from (4.4) and (4.5) that

$$
\begin{aligned}
\forall \boldsymbol{v}_{h} \in \mathbb{V}_{h}, \quad a\left(\tilde{\boldsymbol{u}}_{0 h}-\boldsymbol{w}_{h}, \boldsymbol{v}_{h}\right)= & a\left(\boldsymbol{u}_{0}-\boldsymbol{w}_{h}, \boldsymbol{v}_{h}\right)+a\left(\boldsymbol{u}_{b}-\boldsymbol{u}_{b h}, \boldsymbol{v}_{h}\right)-a\left(\tilde{\boldsymbol{u}}_{h}, \boldsymbol{v}_{h}\right) \\
& +\tilde{b}\left(\boldsymbol{v}_{h}, p-r_{h}\right)+\int_{\Gamma}\left(\left(\boldsymbol{v}_{h \mid \Omega_{P}}-\boldsymbol{v}_{h \mid \Omega_{F}}\right) \cdot \boldsymbol{n}\right)(\boldsymbol{\tau}) p_{\mid \Omega_{P}}(\boldsymbol{\tau}) \mathrm{d} \boldsymbol{\tau} .
\end{aligned}
$$

Since $\tilde{\boldsymbol{u}}_{0 h}-\boldsymbol{w}_{h}$ belongs to $\mathbb{V}_{h}$, we now use the ellipticity property (3.38) of the form $a(\cdot, \cdot)$ on $\mathbb{V}_{h}$. When combined with several Cauchy-Schwarz inequalities, this yields

$$
\begin{aligned}
\left\|\tilde{\boldsymbol{u}}_{0 h}-\boldsymbol{w}_{h}\right\|_{X(\Omega)} \leq & c\left(\left\|\boldsymbol{u}_{0}-\boldsymbol{w}_{h}\right\|_{X(\Omega)}+\left\|\boldsymbol{u}_{b}-\boldsymbol{u}_{b h}\right\|_{X(\Omega)}+\left\|\tilde{\boldsymbol{u}}_{h}\right\|_{X(\Omega)}\right. \\
& \left.+\left\|p-r_{h}\right\|_{L^{2}(\Omega)}+\sup _{\boldsymbol{v}_{h} \in \mathbb{X}_{0 h}} \frac{\int_{\Gamma}\left(\left(\boldsymbol{v}_{h \mid \Omega_{P}}-\boldsymbol{v}_{h \mid \Omega_{F}}\right) \cdot \boldsymbol{n}\right)(\boldsymbol{\tau}) p_{\mid \Omega_{P}}(\boldsymbol{\tau}) \mathrm{d} \boldsymbol{\tau}}{\left\|\boldsymbol{v}_{h}\right\|_{X(\Omega)}}\right) .
\end{aligned}
$$

Combining this with (4.3) and using a further triangle inequality lead to (4.2).

In the right-hand side of (4.2), the first two terms represent the approximation error. The next two ones are issued from the treatment of the Dirichlet boundary conditions. The last term represents the consistency error and is due to the nonconformity of the discretization.

Lemma 4.2. If the assumptions of Lemma 4.1 are satisfied, the following estimate holds between the solution $\left(\boldsymbol{u}_{0}, p\right)$ of problem (2.18) and the solution $\left(\boldsymbol{u}_{0 h}, p_{h}\right)$ of problem (3.36)

$$
\begin{aligned}
\left\|p-p_{h}\right\|_{L^{2}(\Omega)} \leq c\left(\inf _{\boldsymbol{w}_{h} \in \mathbb{V}_{h}}\left\|\boldsymbol{u}_{0}-\boldsymbol{w}_{h}\right\|_{X(\Omega)}\right. & +\inf _{r_{h} \in \mathbb{M}_{h}}\left\|p-r_{h}\right\|_{L^{2}(\Omega)} \\
+\left\|\boldsymbol{u}_{b}-\boldsymbol{u}_{b h}\right\|_{X(\Omega)}+\sup _{q_{h} \in \mathbb{M}_{h}} \frac{\tilde{b}\left(\boldsymbol{u}_{b h}, q_{h}\right)}{\left\|q_{h}\right\|_{L^{2}(\Omega)}} & \\
& \left.+\sup _{\boldsymbol{v}_{h} \in \mathbb{X}_{0 h}} \frac{\int_{\Gamma}\left(\left(\boldsymbol{v}_{h \mid \Omega_{P}}-\boldsymbol{v}_{h \mid \Omega_{F}}\right) \cdot \boldsymbol{n}\right)(\boldsymbol{\tau}) p_{\mid \Omega_{P}}(\boldsymbol{\tau}) \mathrm{d} \boldsymbol{\tau}}{\left\|\boldsymbol{v}_{h}\right\|_{X(\Omega)}}\right) .
\end{aligned}
$$

Proof. The same arguments as in the previous proof yield, for any function $r_{h}$ in $\mathbb{M}_{h}$,

$$
\begin{aligned}
\forall \boldsymbol{v}_{h} \in \mathbb{X}_{0 h}, \quad \tilde{b}\left(\boldsymbol{v}_{h}, p_{h}-r_{h}\right)= & a\left(\boldsymbol{u}_{0}-\boldsymbol{u}_{0 h}, \boldsymbol{v}_{h}\right)+a\left(\boldsymbol{u}_{b}-\boldsymbol{u}_{b h}, \boldsymbol{v}_{h}\right) \\
& +\tilde{b}\left(\boldsymbol{v}_{h}, p-r_{h}\right)+\int_{\Gamma}\left(\left(\boldsymbol{v}_{h \mid \Omega_{P}}-\boldsymbol{v}_{h \mid \Omega_{F}}\right) \cdot \boldsymbol{n}\right)(\boldsymbol{\tau}) p_{\mid \Omega_{P}}(\boldsymbol{\tau}) \mathrm{d} \boldsymbol{\tau} .
\end{aligned}
$$

So the desired estimate follows from the inf-sup condition (3.41) combined with several Cauchy-Schwarz inequalities, estimate (4.2) and a further triangle inequality.

We now evaluate the approximation errors. The distance of the pressure to the space $\mathbb{M}_{h}$ is bounded in a completely standard way, see [9], Chap. IX, Th. 2.1, for instance: If $p_{\mid \Omega_{P}}$ belongs to $H^{1}\left(\Omega_{P}\right)$ (which is always true, see Prop. 2.8) and $p_{\mid \Omega_{F}}$ belongs to $H^{s_{F}}\left(\Omega_{F}\right), 0 \leq s_{F} \leq 1$,

$$
\inf _{r_{h} \in \mathbb{M}_{h}}\left\|p-r_{h}\right\|_{L^{2}(\Omega)} \leq c\left(h_{P}\|p\|_{H^{1}\left(\Omega_{P}\right)}+h_{F}^{s_{F}}\|p\|_{H^{s_{F}\left(\Omega_{F}\right)}}\right) .
$$


To estimate the distance of $\boldsymbol{u}$ to $\mathbb{V}_{h}$, we first use an argument due to [24], Chapter II, formula (1.16): Since $\boldsymbol{u}_{0}$ belongs to $V(\Omega)$, it follows from the inf-sup condition (3.41) that

$$
\inf _{\boldsymbol{w}_{h} \in \mathbb{V}_{h}}\left\|\boldsymbol{u}_{0}-\boldsymbol{w}_{h}\right\|_{X(\Omega)} \leq c \inf _{\boldsymbol{w}_{h} \in \mathbb{X}_{0 h}}\left\|\boldsymbol{u}_{0}-\boldsymbol{w}_{h}\right\|_{X(\Omega)} .
$$

Lemma 4.3. The following estimate holds for any function $\boldsymbol{u}_{0}$ in $V(\Omega)$ such that $\boldsymbol{u}_{0 \mid \Omega_{P}}$ belongs to $H^{s_{P}}\left(\Omega_{P}\right)^{d}$, $0<s_{P} \leq 1$, and $\boldsymbol{u}_{0 \mid \Omega_{F}}$ belongs to $H^{s_{F}+1}\left(\Omega_{F}\right)^{d}, 0 \leq s_{F} \leq 1$,

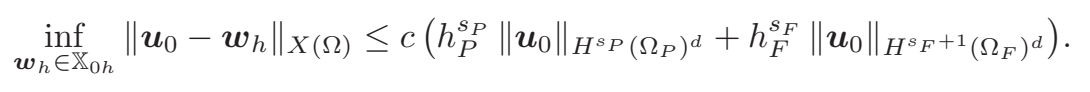

Proof. The construction of the function $\boldsymbol{w}_{h}$ is performed in two steps.

1) We first set

$$
\left.\boldsymbol{w}_{h}^{\sharp}\right|_{\Omega_{P}}=\Pi_{h}^{R T} \boldsymbol{u}_{0},\left.\quad \boldsymbol{w}_{h}^{\sharp}\right|_{\Omega_{F}}=\mathcal{R}_{h} \boldsymbol{u}_{0},
$$

where the Clément regularization operator $\mathcal{R}_{h}$ is introduced in Section 3, see (3.40). Since both $\boldsymbol{u}_{0}$ and $\boldsymbol{w}_{h}^{\sharp}$ are divergence-free on $\Omega_{P}$, we have

$$
\left\|\boldsymbol{u}_{0}-\boldsymbol{w}_{h}^{\sharp}\right\|_{H\left(\operatorname{div}, \Omega_{P}\right)}=\left\|\boldsymbol{u}_{0}-\boldsymbol{w}_{h}^{\sharp}\right\|_{L^{2}\left(\Omega_{P}\right)^{d}} .
$$

Then, relying on the fact that $\Pi_{h}^{R T}$ preserves the constants on each $K$ in $\mathcal{T}_{h}^{P}$ and combining (3.19) with the approximation properties of this constant leads to

$$
\left\|\boldsymbol{u}_{0}-\boldsymbol{w}_{h}^{\sharp}\right\|_{H\left(\operatorname{div}, \Omega_{P}\right)} \leq c h_{P}^{s_{P}}\left\|\boldsymbol{u}_{0}\right\|_{H^{s_{P}}\left(\Omega_{P}\right)^{d}} .
$$

On the other hand, we derive from the approximation properties of the operator $\mathcal{R}_{h}$, see [9], Chap. IX, Th. 3.11, that

$$
\left\|\boldsymbol{u}_{0}-\boldsymbol{w}_{h}^{\sharp}\right\|_{H^{1}\left(\Omega_{F}\right)^{d}} \leq c h_{F}^{s_{F}}\left\|\boldsymbol{u}_{0}\right\|_{H^{s_{F}+1}\left(\Omega_{F}\right)^{d}} .
$$

2) For the functions $\varphi_{e}$ introduced in (3.17), we now set

$$
\left.\boldsymbol{w}_{h}^{b}\right|_{\Omega_{P}}=\sum_{e \in \mathcal{E}_{h}^{P, \Gamma}}\left(\int_{e}\left(\left(\boldsymbol{w}_{h \mid \Omega_{F}}^{\sharp}-\boldsymbol{w}_{h \mid \Omega_{P}}^{\sharp}\right) \cdot \boldsymbol{n}\right)(\boldsymbol{\tau}) \mathrm{d} \boldsymbol{\tau}\right) \boldsymbol{\varphi}_{e},\left.\quad \boldsymbol{w}_{h}^{b}\right|_{\Omega_{F}}=\mathbf{0} .
$$

The arguments for evaluating $\left\|\boldsymbol{w}_{h}^{b}\right\|_{X(\Omega)}$ are nearly the same as in the proof of Lemma 3.8: Combining (3.30) with (3.12) and a Cauchy-Schwarz inequality yields

$$
\left\|\boldsymbol{w}_{h}^{b}\right\|_{X(\Omega)} \leq c\left(\sum_{e \in \mathcal{E}_{h}^{P, \Gamma}} h_{e}^{-1}\left\|\boldsymbol{u}_{0}-\boldsymbol{w}_{h \mid \Omega_{F}}^{\sharp}\right\|_{L^{2}(e)}^{2}\right)^{\frac{1}{2}} .
$$

We refer to [9], Chap. IX, Cor. 3.12, for the following result: On each element $e^{\prime}$ of $\mathcal{E}_{h}^{F, \Gamma}$,

$$
\left\|\boldsymbol{u}_{0}-\mathcal{R}_{h} \boldsymbol{u}_{0}\right\|_{L^{2}\left(e^{\prime}\right)} \leq c h_{e^{\prime}}^{s_{F}+\frac{1}{2}}\left\|\boldsymbol{u}_{0}\right\|_{H^{s_{F}+1}\left(\Delta_{e^{\prime}}\right)^{d}}
$$

where $\Delta_{e^{\prime}}$ is the union of elements $\kappa$ of $\mathcal{T}_{h}^{F}$ such that $e^{\prime} \cap \kappa$ is not empty. Using this estimate for all $e^{\prime}$ such that $e \cap e^{\prime}$ has a positive measure leads to, owing to Lemma 3.7,

$$
\left\|\boldsymbol{w}_{h}^{b}\right\|_{X(\Omega)} \leq c h_{F}^{s_{F}}\left\|\boldsymbol{u}_{0}\right\|_{H^{s_{F}+1}\left(\Omega_{F}\right)^{d}} .
$$


To conclude, we note that the function $\boldsymbol{w}_{h}=\boldsymbol{w}_{h}^{\sharp}+\boldsymbol{w}_{h}^{b}$ belongs to $X_{0 h}$. Estimate (4.9) is then derived from (4.10), (4.11) and (4.12).

Estimating the consistency error requires the orthogonal projection operator from $L^{2}(\Gamma)$ onto $\mathbb{W}_{h}$, that we denote by $\pi_{h}^{\Gamma}$.

Lemma 4.4. The following estimate holds for any function $p$ in $L^{2}(\Omega)$ such that $p_{\mid \Omega_{P}}$ belongs to $H^{s_{P}+1}\left(\Omega_{P}\right)$, $0 \leq s_{P} \leq \frac{1}{2}$

$$
\sup _{\boldsymbol{v}_{h} \in \mathbb{X}_{0 h}} \frac{\int_{\Gamma}\left(\left(\boldsymbol{v}_{h \mid \Omega_{P}}-\boldsymbol{v}_{h \mid \Omega_{F}}\right) \cdot \boldsymbol{n}\right)(\boldsymbol{\tau}) p_{\mid \Omega_{P}}(\boldsymbol{\tau}) \mathrm{d} \boldsymbol{\tau}}{\left\|\boldsymbol{v}_{h}\right\|_{X(\Omega)}} \leq c h_{P}^{s_{P}+1}\|p\|_{H^{s_{P}+1}\left(\Omega_{P}\right)} .
$$

Proof. It follows from the matching conditions (3.7) that, for each $e$ in $\mathcal{E}_{h}^{P, \Gamma}$,

$$
\int_{\Gamma}\left(\left(\boldsymbol{v}_{h \mid \Omega_{P}}-\boldsymbol{v}_{h \mid \Omega_{F}}\right) \cdot \boldsymbol{n}\right)(\boldsymbol{\tau}) p_{\mid \Omega_{P}}(\boldsymbol{\tau}) \mathrm{d} \boldsymbol{\tau}=\int_{\Gamma}\left(\left(\boldsymbol{v}_{h \mid \Omega_{P}}-\boldsymbol{v}_{h \mid \Omega_{F}}\right) \cdot \boldsymbol{n}\right)(\boldsymbol{\tau})\left(p_{\mid \Omega_{P}}-\pi_{h}^{\Gamma} p_{\mid \Omega_{P}}\right)(\boldsymbol{\tau}) \mathrm{d} \boldsymbol{\tau} .
$$

Moreover, since the normal trace of $\boldsymbol{v}_{h \mid \Omega_{P}}$ on $\Gamma$ belongs to $\mathbb{W}_{h}$ for any $\boldsymbol{v}_{h}$ in $\mathbb{X}_{0 h}$, this gives

$$
\int_{\Gamma}\left(\left(\boldsymbol{v}_{h \mid \Omega_{P}}-\boldsymbol{v}_{h \mid \Omega_{F}}\right) \cdot \boldsymbol{n}\right)(\boldsymbol{\tau}) p_{\mid \Omega_{P}}(\boldsymbol{\tau}) \mathrm{d} \boldsymbol{\tau}=-\int_{\Gamma}\left(\boldsymbol{v}_{h \mid \Omega_{F}} \cdot \boldsymbol{n}\right)(\boldsymbol{\tau})\left(p_{\mid \Omega_{P}}-\pi_{h}^{\Gamma} p_{\mid \Omega_{P}}\right)(\boldsymbol{\tau}) \mathrm{d} \boldsymbol{\tau} .
$$

This yields

$$
\frac{\int_{\Gamma}\left(\left(\boldsymbol{v}_{h \mid \Omega_{P}}-\boldsymbol{v}_{h \mid \Omega_{F}}\right) \cdot \boldsymbol{n}\right)(\boldsymbol{\tau}) p_{\mid \Omega_{P}}(\boldsymbol{\tau}) \mathrm{d} \boldsymbol{\tau}}{\left\|\boldsymbol{v}_{h}\right\|_{X(\Omega)}} \leq \frac{\left\|\boldsymbol{v}_{h \mid \Omega_{F}}\right\|_{H^{\frac{1}{2}}(\Gamma) d}\left\|p_{\mid \Omega_{P}}-\pi_{h}^{\Gamma} p_{\mid \Omega_{P}}\right\|_{H^{-\frac{1}{2}}(\Gamma)}}{\left\|\boldsymbol{v}_{h}\right\|_{X(\Omega)}}
$$

whence, by applying the trace theorem on $\Gamma$,

$$
\frac{\int_{\Gamma}\left(\left(\boldsymbol{v}_{h \mid \Omega_{P}}-\boldsymbol{v}_{h \mid \Omega_{F}}\right) \cdot \boldsymbol{n}\right)(\boldsymbol{\tau}) p_{\mid \Omega_{P}}(\boldsymbol{\tau}) \mathrm{d} \boldsymbol{\tau}}{\left\|\boldsymbol{v}_{h}\right\|_{X(\Omega)}} \leq c\left\|p_{\mid \Omega_{P}}-\pi_{h}^{\Gamma} p_{\mid \Omega_{P}}\right\|_{H^{-\frac{1}{2}}(\Gamma)} .
$$

The standard duality argument

$$
\left\|p_{\mid \Omega_{P}}-\pi_{h}^{\Gamma} p_{\mid \Omega_{P}}\right\|_{H^{-\frac{1}{2}}(\Gamma)}=\sup _{\varphi \in H^{\frac{1}{2}}(\Gamma)} \frac{\int_{\Gamma}\left(p_{\mid \Omega_{P}}-\pi_{h}^{\Gamma} p_{\mid \Omega_{P}}\right)(\boldsymbol{\tau})\left(\varphi-\pi_{h}^{\Gamma} \varphi\right)(\boldsymbol{\tau}) \mathrm{d} \boldsymbol{\tau}}{\|\varphi\|_{H^{\frac{1}{2}}(\Gamma)}},
$$

combined with the approximation properties of the operator $\pi_{h}^{\Gamma}$, see [9], Chap. IX, Th. 2.1, leads to

$$
\left\|p_{\mid \Omega_{P}}-\pi_{h}^{\Gamma} p_{\mid \Omega_{P}}\right\|_{H^{-\frac{1}{2}(\Gamma)}} \leq c h_{P}^{s_{P}+1}\left\|p_{\mid \Omega_{P}}\right\|_{H^{s_{P}+\frac{1}{2}}(\Gamma)},
$$

whence the desired result.

The five terms in the right-hand side of (4.2) and (4.6) are bounded in (4.8) and Lemma 4.3, (4.7), Corollary 3.10 and Lemma 4.4, respectively. When combining this with (4.1) and using once more Corollary 3.10, we derive the a priori error estimate. We recall that the parameters $\lambda_{P}$ and $\lambda_{F}$ have been introduced in Notation 3.9 . 
Theorem 4.5. Assume that $\Gamma_{F}$ has a positive measure in $\partial \Omega_{F}$ and moreover that

(i) the data $(k, \boldsymbol{g})$ belong to $H^{\sigma_{P}}\left(\Gamma_{P}\right) \times H^{\sigma_{F}}\left(\Gamma_{F}\right)^{d}, \sigma_{P}>-\frac{1}{2}$ and $\sigma_{F}>\frac{d-1}{2}$;

(ii) the solution $\left(\boldsymbol{u}_{0}, p\right)$ of problem $(2.18)$ is such that $\left(\boldsymbol{u}_{0 \mid \Omega_{P}}, p_{\mid \Omega_{P}}\right)$ belongs to $H^{s_{P}}\left(\Omega_{P}\right)^{d} \times H^{1}\left(\Omega_{P}\right)$, $0<s_{P} \leq 1$, and $\left(\boldsymbol{u}_{0 \mid \Omega_{F}}, p_{\mid \Omega_{F}}\right)$ belongs to $H^{s_{F}+1}\left(\Omega_{F}\right)^{d} \times H^{s_{F}}\left(\Omega_{F}\right), 0 \leq s_{F} \leq 1$.

Then the following a priori error estimate holds between the solution $(\boldsymbol{u}, p)$ of problem (2.6)-(2.7) and the solution $\left(\boldsymbol{u}_{h}, p_{h}\right)$ of problem (3.9)-(3.10)

$$
\begin{aligned}
& \left\|\boldsymbol{u}-\boldsymbol{u}_{h}\right\|_{X(\Omega)}+\left\|p-p_{h}\right\|_{L^{2}(\Omega)} \leq c\left(h_{P}^{s_{P}}\left(\left\|\boldsymbol{u}_{0}\right\|_{H^{s_{P}\left(\Omega_{P}\right)^{d}}}+\|p\|_{H^{1}\left(\Omega_{P}\right)}\right)+h_{F}^{s_{F}}\left(\left\|\boldsymbol{u}_{0}\right\|_{H^{s_{F}+1}\left(\Omega_{F}\right)^{d}}+\|p\|_{H^{s_{F}\left(\Omega_{F}\right)}}\right)\right. \\
& +\left(h_{P}^{\min \left\{\sigma_{P}+\frac{1}{2}, \lambda_{P}\right\}}+h_{F}^{\min \left\{\sigma_{F}-\frac{1}{2}, \lambda_{F}\right\}}\right)\left(\|k\|_{H^{\sigma_{P}\left(\Gamma_{P}\right)}}+\|\boldsymbol{g}\|_{\left.H^{\sigma_{F}\left(\Gamma_{F}\right)^{d}}\right)}\right) .
\end{aligned}
$$

The statement of Theorem 4.5 is rather complex. Note anyhow that:

- In the case of zero boundary conditions $k$ and $\boldsymbol{g}$, estimate (4.14) can be written more simply as

$$
\begin{aligned}
\left\|\boldsymbol{u}-\boldsymbol{u}_{h}\right\|_{X(\Omega)}+\left\|p-p_{h}\right\|_{L^{2}(\Omega)} & \\
& \leq c\left(h_{P}^{s_{P}}\left(\|\boldsymbol{u}\|_{H^{s_{P}}\left(\Omega_{P}\right)^{d}}+\|p\|_{H^{1}\left(\Omega_{P}\right)}\right)+h_{F}^{s_{F}}\left(\|\boldsymbol{u}\|_{H^{s_{F}}+1\left(\Omega_{F}\right)^{d}}+\|p\|_{H^{s_{F}}\left(\Omega_{F}\right)}\right)\right) .
\end{aligned}
$$

This last estimate is fully optimal: Indeed, for a smooth solution $(\boldsymbol{u}, p)$, the error behaves like $h_{P}+h_{F}$.

- In the general case, the order of convergence depends on the parameters $\lambda_{P}$ and $\lambda_{F}$. So the order 1 is only obtained when $\Gamma_{a P}$ is empty and both $\Omega_{P}$ and $\Omega_{F}$ are convex, for smooth data and solutions. When the regularity of $(\boldsymbol{u}, p)$ is unknown, the order of convergence is given by Proposition 2.8 and, for instance, is always larger than $1 / 4$ in dimension $d=2$. Moreover, a different analysis (relying on the construction of an approximation of $\boldsymbol{u}$ in $\mathbb{X}_{h}$ satisfying the boundary conditions (3.9), which requires the continuity of $\left.\boldsymbol{u}_{\mid \Omega_{F}}\right)$, yields that, there also, for a smooth solution $(\boldsymbol{u}, p)$, the error behaves like $h_{P}+h_{F}$.

To conclude, it can be observed that, in all cases and for smooth enough data $(k, \boldsymbol{g})$, the convergence of the discretization results from Theorem 4.5.

\section{A POSTERIORI ERROR ESTIMATES}

Some further notation are needed to define the error indicators. For each $K$ in $\mathcal{T}_{h}^{P}$, we denote

- by $\mathcal{E}_{K}$ the set of edges $(d=2)$ or faces $(d=3)$ of $K$ which are not contained in $\partial \Omega_{P}$;

- by $\mathcal{E}_{K}^{a P}$ the set of edges $(d=2)$ or faces $(d=3)$ of $K$ which are contained in $\bar{\Gamma}_{a P}$. For each $K$ in $\mathcal{T}_{h}^{F}$, we denote

- by $\mathcal{E}_{K}$ the set of edges $(d=2)$ or faces $(d=3)$ of $K$ which are not contained in $\partial \Omega_{F}$;

- by $\mathcal{E}_{K}^{a F}$ the set of edges $(d=2)$ or faces $(d=3)$ of $K$ which are contained in $\bar{\Gamma}_{a F}$.

For each $e$ in any of the $\mathcal{E}_{K}$ and also in $\mathcal{E}_{h}^{P, \Gamma}$, we agree to denote by $[\cdot]_{e}$ the jump through $e$ (making its sign precise is not necessary). We also denote by $h_{e}$ the length $(d=2)$ or diameter $(d=3)$ of $e$.

We need a further notation for some global sets:

- $\mathcal{E}_{h}^{a P}$ is the set of edges or faces of elements of $\mathcal{T}_{h}^{P}$ which are contained in $\bar{\Gamma}_{a P}$;

- $\mathcal{E}_{h}^{P}$ is the set of all other edges or faces of elements of $\mathcal{T}_{h}^{P}$.

With each element $K$ of $\mathcal{T}_{h}^{F}$ and each edge $e$ of $K$, we associate the quantities $\gamma_{K}$ and $\gamma_{e}$ equal to 1 if $K$ or $e$, respectively, intersects $\bar{\Gamma} \backslash \bar{\Gamma}_{F}$ and to zero otherwise.

We introduce the space $\mathbb{Z}_{h}$ of functions in $L^{2}(\Omega)^{d}$ such that their restrictions to each $K$ in $\mathcal{T}_{h}^{P}$ or in $\mathcal{T}_{h}^{F}$ is constant. Similarly, we denote by $\mathbb{Z}_{h}^{F}$ the space of functions in $L^{2}\left(\Gamma_{a F}\right)^{d}$ such that their restriction to each $e$ in $\mathcal{E}_{K}^{a F}, K \in \mathcal{T}_{h}^{F}$, is constant. Indeed, we consider an approximation $\boldsymbol{f}_{h}$ of $\boldsymbol{f}$ in $\mathbb{Z}_{h}$ and an approximation $\boldsymbol{t}_{a h}$ of $\boldsymbol{t}_{a}$ in $\mathbb{Z}_{h}^{F}$. Finally, assuming that the datum $p_{a}$ is continuous on $\bar{\Gamma}_{a P}$, we define $p_{a h}$ as the function which is affine on each $e$ in $\mathcal{E}_{K}^{a P}, K \in \mathcal{T}_{h}^{P}$, and equal to $p_{a}(\boldsymbol{a})$ at all endpoints $(d=2)$ or vertices $(d=3) \boldsymbol{a}$ of these $e$. 
We consider three families of error indicators, related to the error on $\Omega_{P}, \Omega_{F}$ and $\Gamma$, respectively.

- For each $K$ in $\mathcal{T}_{h}^{P}$, the error indicator $\eta_{K}^{P}$ is defined by

$$
\eta_{K}^{P}=\left\|\boldsymbol{f}_{h}-\alpha \boldsymbol{u}_{h}\right\|_{L^{2}(K)^{d}}+\sum_{e \in \mathcal{E}_{K}} h_{e}^{-\frac{1}{2}}\left\|\left[p_{h}\right]_{e}\right\|_{L^{2}(e)}+\sum_{e \in \mathcal{E}_{K}^{a P}} h_{e}^{-\frac{1}{2}}\left\|p_{a h}-p_{h}\right\|_{L^{2}(e)} .
$$

- For each $K$ in $\mathcal{T}_{h}^{F}$, the error indicator $\eta_{K}^{F}$ is defined by

$$
\begin{aligned}
\eta_{K}^{F}= & h_{K}^{1-\gamma_{K}}\left\|\boldsymbol{f}_{h}+\nu \Delta \boldsymbol{u}_{h}\right\|_{L^{2}(K)^{d}}+\sum_{e \in \mathcal{E}_{K}} h_{e}^{\frac{1}{2}-\gamma_{e}}\left\|\left[\nu \partial_{n} \boldsymbol{u}_{h}-p_{h} \boldsymbol{n}\right]_{e}\right\|_{L^{2}(e)^{d}} \\
& +\sum_{e \in \mathcal{E}_{K}^{a F}} h_{e}^{\frac{1}{2}-\gamma_{e}}\left\|\boldsymbol{t}_{a h}-\nu \partial_{n} \boldsymbol{u}_{h}+p_{h} \boldsymbol{n}\right\|_{L^{2}(e)^{d}}+\left\|\operatorname{div} \boldsymbol{u}_{h}\right\|_{L^{2}(K)} .
\end{aligned}
$$

- For each $e$ in $\mathcal{E}_{h}^{P, \Gamma}$, the error indicator $\eta_{e}^{\Gamma}$ is defined by

$$
\eta_{e}^{\Gamma}=\left\|\left(p_{h} \boldsymbol{n}\right)_{\mid \Omega_{P}}+\left(\nu \partial_{n} \boldsymbol{u}_{h}-p_{h} \boldsymbol{n}\right)_{\mid \Omega_{F}}\right\|_{L^{2}(e)^{d}}+h_{e}^{-\frac{1}{2}}\left\|\left[\boldsymbol{u}_{h} \cdot \boldsymbol{n}\right]_{e}\right\|_{L^{2}(e)} .
$$

It is readily checked that these indicators are easy to compute once the discrete solution $\left(\boldsymbol{u}_{h}, p_{h}\right)$ is known. Moreover, they are all of residual type. Note also that only the second term in the $\eta_{e}^{\Gamma}$ comes from the nonconformity of the discretization.

In order to perform the a posteriori analysis, we first recall a useful argument due to R. Verfürth, see [8], Section 4 for instance. Indeed, we now set

$$
\forall U=(\boldsymbol{u}, p) \in Y(\Omega) \times L^{2}(\Omega), \quad \forall V=(\boldsymbol{v}, q) \in Y(\Omega) \times L^{2}(\Omega), \quad \mathcal{A}(U, V)=a(\boldsymbol{u}, \boldsymbol{v})+\tilde{b}(\boldsymbol{v}, p)+\tilde{b}(\boldsymbol{u}, q),
$$

where $Y(\Omega)$ stands for the space

$$
Y(\Omega)=\left\{\boldsymbol{v} \in L^{2}(\Omega)^{d} ; \boldsymbol{v}_{\mid \Omega_{P}} \in H\left(\operatorname{div}, \Omega_{P}\right) \text { and } v_{\mid \Omega_{F}} \in H^{1}\left(\Omega_{F}\right)^{d}\right\},
$$

of course equipped with the norm $\|\cdot\|_{X(\Omega)}$. Indeed, it is readily checked that the form $\mathcal{A}(\cdot, \cdot)$ is continuous on $\left(Y(\Omega) \times L^{2}(\Omega)\right) \times\left(Y(\Omega) \times L^{2}(\Omega)\right)$. Moreover, the following property is a direct consequence of the ellipticity property (2.20) and of the inf-sup condition (2.23), see [24], Chapter I, Lemma 4.1.

Lemma 5.1. If $\Gamma_{F}$ has a positive measure in $\partial \Omega_{F}$, there exists a constant $\gamma>0$ such that the following inf-sup condition holds

$$
\forall U \in X_{0}(\Omega) \times L^{2}(\Omega), \quad \sup _{V \in X_{0}(\Omega) \times L^{2}(\Omega)} \frac{\mathcal{A}(U, V)}{\|V\|_{X(\Omega) \times L^{2}(\Omega)}} \geq \gamma\|U\|_{X(\Omega) \times L^{2}(\Omega)} .
$$

Unfortunately, the function $\boldsymbol{u}-\boldsymbol{u}_{h}$ does not belong to $X_{0}(\Omega)$ and even not to $X(\Omega)$, so that we cannot apply directly Lemma 5.1 to the residual equation satisfied by $U-U_{h}$. The idea consists in building a conforming approximation of $\boldsymbol{u}_{h}$, namely an approximation which belongs to $X(\Omega)$ (see [12], Lem. 5.4 for a similar argument and [18] for a general analysis in a different context).

From now on, we call finite element function a function such that its restriction to each element of $\mathcal{T}_{h}^{P}$ or $\mathcal{T}_{h}^{F}$ is a polynomial with degree bounded independently of $h_{P}$ and $h_{F}$. We are led to make a further assumption, which is now standard in the a posteriori analysis of mortar element discretizations (and is stronger than Assumption 3.2). We recall from the previous sections that $\mathcal{E}_{h}^{P, \Gamma}$ and $\mathcal{E}_{h}^{F, \Gamma}$ denote the set of edges $(d=2)$ or faces $(d=3)$ of elements of $\mathcal{T}_{h}^{P}$ or $\mathcal{T}_{h}^{F}$, respectively, which are contained in $\bar{\Gamma}$. 


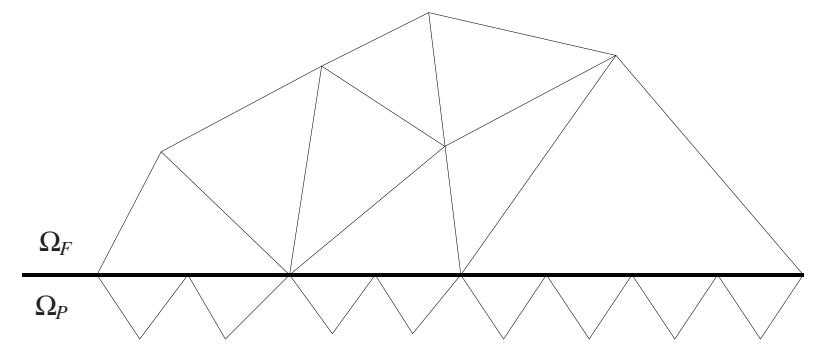

FiguRE 3. Illustration of Assumption 5.2.

Assumption 5.2. Each element $e$ of $\mathcal{E}_{h}^{F, \Gamma}$ is the union of a finite number of elements of $\mathcal{E}_{h}^{P, \Gamma}$, where "finite" means bounded independently of $h_{P}$ and $h_{F}$ (see Fig. 3).

Lemma 5.3. If Assumption 5.2 holds, there exists a finite element function $\boldsymbol{u}_{h}^{*}$ in $X(\Omega)$, still satisfying the boundary conditions (3.9), such that

$$
\left\|\boldsymbol{u}_{h}-\boldsymbol{u}_{h}^{*}\right\|_{X(\Omega)} \leq c\left(\sum_{e \in \mathcal{E}_{h}^{P, \Gamma}} h_{e}^{-1}\left\|\left[\boldsymbol{u}_{h} \cdot \boldsymbol{n}\right]_{e}\right\|_{L^{2}(e)}^{2}\right)^{\frac{1}{2}}
$$

Proof. It follows from Assumption 5.2 that each $e$ in $\mathcal{E}_{h}^{F, \Gamma}$ is the union of edges $e_{i}$ of $\mathcal{E}_{h}^{P, \Gamma}, 1 \leq i \leq I_{e}$, where $I_{e}$ is bounded independently of $h_{P}$ and $h_{F}$ (see Fig. 3). We denote by $\boldsymbol{n}_{e}$ the unit normal vector on $e$, directed from $\Omega_{P}$ to $\Omega_{F}$. Let $K_{i}$ be the element of $\mathcal{T}_{h}^{P}$ such that $e_{i}$ is an edge of $K_{i}$, let $\boldsymbol{b}_{i}$ denote the vertex of $K_{i}$ which does not belong to $e_{i}$. Thus, it can be checked that the function $\varphi_{e_{i}}$ introduced in (3.17) (with obvious notation) is equal to $\frac{\boldsymbol{x}-\boldsymbol{b}_{i}}{d \operatorname{meas}\left(K_{i}\right)}$ on $K_{i}$ and to zero elsewhere. Its normal trace on $e_{i}$ is constant, equal to $\frac{1}{\text { meas }\left(e_{i}\right)}$. On the other hand, it must be observed that

- on each $e$ in $\mathcal{E}_{h}^{F . \Gamma}$ which is an edge or a face of $K, \boldsymbol{u}_{h \mid e}$ is the trace of a function in $\mathcal{P}_{B R}(K)$, which we denote by $\boldsymbol{u}_{h}^{e}$ for a while;

- on each edge $e_{i}, \boldsymbol{u}_{h \mid \Omega_{P}}$ is equal to $\alpha_{i} \boldsymbol{\varphi}_{e_{i}}$ for a constant $\alpha_{i}$.

Next, we set

$$
w_{e_{i}}=\mathcal{R}_{e_{i}}\left(\boldsymbol{u}_{h}^{e} \cdot \boldsymbol{n}_{e}\right) \operatorname{meas}\left(e_{i}\right)-\alpha_{i},
$$

where $\mathcal{R}_{e_{i}}$ is a lifting operator from polynomials on $e_{i}$ onto polynomials on $K_{i}$, constructed by affine transformation from a fixed lifting operator on a reference triangle, and we define

$$
\boldsymbol{u}_{h}^{*}=\boldsymbol{u}_{h}+\sum_{e \in \mathcal{E}_{h}^{F}, \Gamma} \sum_{i=1}^{I_{e}} w_{e_{i}} \boldsymbol{\varphi}_{e_{i}}
$$

Since $\boldsymbol{u}_{h}^{*}$ coincides with $\boldsymbol{u}_{h}$ in $\Omega_{F}$, the function $\boldsymbol{u}_{h}^{*}-\boldsymbol{u}_{h}$ has its support contained in $\Omega_{P} \cup \Gamma$. Moreover, since the normal trace of $\varphi_{e_{i}}$ vanishes on all edges of triangles or all faces of tetrahedra $K_{i}$ that are not contained in $\Gamma, \boldsymbol{u}_{h}^{*}-\boldsymbol{u}_{h}$ belongs to $H\left(\operatorname{div}, \Omega_{P}\right)$ and $\boldsymbol{u}_{h}^{*}$ satisfies the boundary conditions (3.9). Finally, the jump of $\boldsymbol{u}_{h}^{*} \cdot \boldsymbol{n}_{e}$ on each $e$ in $\mathcal{E}_{h}^{P, \Gamma}$ is equal to zero, so that $\boldsymbol{u}_{h}^{*}$ belongs to $X(\Omega)$.

In order to prove a bound for $\left\|\boldsymbol{u}_{h}-\boldsymbol{u}_{h}^{*}\right\|_{H\left(\operatorname{div}, \Omega_{P}\right)}$, we write

$$
\left\|w_{e_{i}} \boldsymbol{\varphi}_{e_{i}}\right\|_{H\left(\operatorname{div}, K_{i}\right)} \leq\left\|w_{e_{i}}\right\|_{H^{1}\left(K_{i}\right)}\left\|\boldsymbol{\varphi}_{e_{i}}\right\|_{L^{\infty}\left(K_{i}\right)^{d}}+\left\|w_{e_{i}}\right\|_{L^{2}\left(K_{i}\right)}\left\|\operatorname{div} \boldsymbol{\varphi}_{e_{i}}\right\|_{L^{\infty}\left(K_{i}\right)} .
$$

Next, we use the estimates

$$
\left\|\varphi_{e_{i}}\right\|_{L^{\infty}\left(K_{i}\right)^{d}} \leq c h_{e_{i}}^{1-d}, \quad\left\|\operatorname{div} \varphi_{e_{i}}\right\|_{L^{\infty}\left(K_{i}\right)} \leq c h_{e_{i}}^{-d}
$$


and, noting that the trace of $w_{e_{i}}$ on $e_{i}$ is equal to $\left[\boldsymbol{u}_{h} \cdot \boldsymbol{n}\right]_{e}$ meas $\left(e_{i}\right)$, we obtain by switching to the reference triangle

Combining all this yields

$$
\left\|w_{e_{i}}\right\|_{H^{1}\left(K_{i}\right)}+h_{e_{i}}^{-1}\left\|w_{e_{i}}\right\|_{L^{2}\left(K_{i}\right)} \leq h_{e_{i}}^{d-1-\frac{1}{2}}\left\|\left[\boldsymbol{u}_{h} \cdot \boldsymbol{n}\right]_{e}\right\|_{L^{2}\left(e_{i}\right)} .
$$

$$
\left\|w_{e_{i}} \boldsymbol{\varphi}_{e_{i}}\right\|_{H\left(\operatorname{div}, K_{i}\right)} \leq c h_{e_{i}}^{-\frac{1}{2}}\left\|\left[\boldsymbol{u}_{h} \cdot \boldsymbol{n}\right]_{e}\right\|_{L^{2}\left(e_{i}\right)} .
$$

Summing the square of this estimate on the $K_{i}$ gives (5.7).

For a different reason, mainly due to the lack of regularity of the normal trace of functions in $H\left(\operatorname{div}, \Omega_{P}\right)$, we also need an approximation $p_{h}^{*}$ of $p_{h}$ in $H^{1}\left(\Omega_{P}\right)$. The construction of such a function is standard, see [2], Theorem 4.7. Therefore, we only give an abridged proof of the following lemma.

Lemma 5.4. There exists a finite element function $p_{h}^{*}$ equal to $p_{h}$ on $\Omega_{F}$ and to $p_{a h}$ on $\Gamma_{a P}$, such that $p_{h \mid \Omega_{P}}^{*}$ belongs to $H^{1}\left(\Omega_{P}\right)$ and satisfies

$$
\begin{aligned}
\left\|p_{h}-p_{h}^{*}\right\|_{L^{2}\left(\Omega_{P}\right)} & \leq c\left(\sum_{e \in \mathcal{E}_{h}^{P}} h_{e}\left\|\left[p_{h}\right]_{e}\right\|_{L^{2}(e)}^{2}+\sum_{e \in \mathcal{E}_{h}^{a P}} h_{e}\left\|p_{h}-p_{a h}\right\|_{L^{2}(e)}^{2}\right)^{\frac{1}{2}}, \\
\left|p_{h}^{*}\right|_{H^{1}\left(\Omega_{P}\right)} & \leq c\left(\sum_{e \in \mathcal{E}_{h}^{P}} h_{e}^{-1}\left\|\left[p_{h}\right]_{e}\right\|_{L^{2}(e)}^{2}+\sum_{e \in \mathcal{E}_{h}^{a P}} h_{e}^{-1}\left\|p_{h}-p_{a h}\right\|_{L^{2}(e)}^{2}\right)^{\frac{1}{2}},
\end{aligned}
$$

and

$$
\left\|p_{h}-p_{h}^{*}\right\|_{L^{2}(\Gamma)} \leq c\left(\sum_{e \in \mathcal{E}_{h}^{P}}\left\|\left[p_{h}\right]_{e}\right\|_{L^{2}(e)}^{2}+\sum_{e \in \mathcal{E}_{h}^{a P}}\left\|p_{h}-p_{a h}\right\|_{L^{2}(e)}^{2}\right)^{\frac{1}{2}} .
$$

Proof. Let $\mathcal{V}_{h}^{P}$ denote the set of all vertices of the elements of $\mathcal{T}_{h}^{P}$. We define $p_{h \mid \Omega_{P}}^{*}$ as the function which is affine on each element $K$ of $\mathcal{T}_{h}^{P}$, equal

- to $p_{a h}(\boldsymbol{a})$ at all vertices $\boldsymbol{a}$ in $\mathcal{V}_{h}^{P}$ which belong to $\bar{\Gamma}_{a P}$;

- and, at all other vertices $\boldsymbol{a}$ in $\mathcal{V}_{h}^{P}$, to the mean value of the $p_{h \mid K}(\boldsymbol{a})$ on all elements $K$ of $\mathcal{T}_{h}^{P}$ which contain $\boldsymbol{a}$.

This leads to the construction of $p_{h}^{*}$ satisfying the properties stated in the lemma. Estimates (5.8) and (5.9) are derived by exactly the same arguments as in [2], Theorem 4.7, see also [12], Lemma 5.4.

Finally, with any function $\boldsymbol{v}$ in $X_{0}(\Omega)$, we associate the function $\boldsymbol{v}_{h}$ equal to $\widetilde{\mathcal{R}}_{h} \boldsymbol{v}$ on $\Omega_{F}$ and to zero on $\Omega_{P}$, where $\widetilde{\mathcal{R}}_{h}$ stands for the modified Clément operator with values in piecewise affine functions which vanish on $\Gamma_{F} \cup \Gamma$. The main interest of this $\boldsymbol{v}_{h}$ is that it belongs to $X_{0}(\Omega)$. The next lemma is derived by using the local approximation properties of the operator $\widetilde{\mathcal{R}}_{h}$ see [9], Chap. IX, Section 3.

Lemma 5.5. For any function $\boldsymbol{v}$ in $X_{0}(\Omega)$, there exists a function $\boldsymbol{v}_{h}$ in $\mathbb{X}_{0 h} \cap X_{0}(\Omega)$, with support in $\Omega_{F}$, such that, for all $K$ in $\mathcal{T}_{h}^{F}$ and all edges or faces e of $K$,

$$
h_{K}^{-\left(1-\gamma_{K}\right)}\left\|\boldsymbol{v}-\boldsymbol{v}_{h}\right\|_{L^{2}(K)^{d}}+h_{e}^{-\left(\frac{1}{2}-\gamma_{e}\right)}\left\|\boldsymbol{v}-\boldsymbol{v}_{h}\right\|_{L^{2}(e)^{d}} \leq c\|\boldsymbol{v}\|_{H^{1}\left(\Delta_{K}\right)},
$$

where $\Delta_{K}$ is the union of the elements of $\mathcal{T}_{h}^{F}$ that intersects $K$.

Setting $U=(\boldsymbol{u}, p), U_{h}=\left(\boldsymbol{u}_{h}, p_{h}\right)$ and $U_{h}^{*}=\left(\boldsymbol{u}_{h}^{*}, p_{h}^{*}\right)$, we observe that the function $U-U_{h}^{*}$ now belongs to $X(\Omega) \times L^{2}(\Omega)$. Moreover, it satisfies

$$
\left(\boldsymbol{u}-\boldsymbol{u}_{h}^{*}\right) \cdot \boldsymbol{n}=k-k_{h} \quad \text { on } \Gamma_{P} \quad \text { and } \quad \boldsymbol{u}_{h}-\boldsymbol{u}_{h}^{*}=\boldsymbol{g}-\boldsymbol{g}_{h} \quad \text { on } \Gamma_{F} .
$$


Thus, applying Lemma 2.3 yields the existence of a divergence-free function $\mathbf{e}_{b}$ in $X(\Omega)$ such that $\boldsymbol{u}-\boldsymbol{u}_{h}^{*}-\mathbf{e}_{b}$ belongs to $X_{0}(\Omega)$ and which satisfies

$$
\left\|\mathbf{e}_{b}\right\|_{X(\Omega)} \leq c\left(\left\|k-k_{h}\right\|_{H^{-\frac{1}{2}}\left(\Gamma_{P}\right)}+\left\|\boldsymbol{g}-\boldsymbol{g}_{h}\right\|_{H^{\frac{1}{2}}\left(\Gamma_{F}\right)^{d}}\right) .
$$

When setting $E_{b}=\left(\mathbf{e}_{b}, 0\right)$, we observe that the function $U-U_{h}^{*}-E_{b}$ now belongs to $X_{0}(\Omega) \times L^{2}(\Omega)$.

Next, we write, for any $V=(\boldsymbol{v}, q)$ in $X_{0}(\Omega) \times L^{2}(\Omega)$,

$$
\begin{aligned}
\mathcal{A}\left(U-U_{h}^{*}-E_{b}, V\right)= & a\left(\boldsymbol{u}_{h}-\boldsymbol{u}_{h}^{*}-\boldsymbol{e}_{b}, \boldsymbol{v}\right)+\tilde{b}\left(\boldsymbol{u}_{h}-\boldsymbol{u}_{h}^{*}, q\right) \\
& +a\left(\boldsymbol{u}-\boldsymbol{u}_{h}, \boldsymbol{v}\right)+\tilde{b}\left(\boldsymbol{v}, p-p_{h}^{*}\right)+\tilde{b}\left(\boldsymbol{u}-\boldsymbol{u}_{h}, q\right) .
\end{aligned}
$$

Denoting by $\boldsymbol{v}_{h}$ the approximation of $\boldsymbol{v}$ exhibited in Lemma 5.5 and setting $V_{h}=\left(\boldsymbol{v}_{h}, 0\right)$, we observe from problems (2.6)-(2.7) and (3.9)-(3.10) (note that $\tilde{b}\left(\boldsymbol{v}_{h}, p_{h}-p_{h}^{*}\right)$ is zero since the intersection of the supports of $\boldsymbol{v}_{h}$ and $p_{h}-p_{h}^{*}$ is empty) that we can subtract $V_{h}$ from the second line in this equation. Thus, we derive by integration by parts the following residual equation

$$
\begin{aligned}
\mathcal{A}\left(U-U_{h}^{*}-E_{b}, V\right)= & a\left(\boldsymbol{u}_{h}-\boldsymbol{u}_{h}^{*}-\boldsymbol{e}_{b}, \boldsymbol{v}\right)+\tilde{b}\left(\boldsymbol{u}_{h}-\boldsymbol{u}_{h}^{*}, q\right) \\
& +\left\langle\mathcal{R}_{P}, V-V_{h}\right\rangle+\left\langle\mathcal{R}_{F}, V-V_{h}\right\rangle+\left\langle\mathcal{R}_{\Gamma}, V-V_{h}\right\rangle+\left\langle\mathcal{F}, V-V_{h}\right\rangle,
\end{aligned}
$$

where the four quantities $\mathcal{R}_{P}, \mathcal{R}_{F}, \mathcal{R}_{\Gamma}$ and $\mathcal{F}$ are given by

$$
\begin{aligned}
&\left\langle\mathcal{R}_{P}, V\right\rangle= \sum_{K \in \mathcal{T}_{h}^{P}}\left(\int_{K}\left(\boldsymbol{f}_{h}-\alpha \boldsymbol{u}_{h}\right)(\boldsymbol{x}) \cdot \boldsymbol{v}(\boldsymbol{x}) \mathrm{d} \boldsymbol{x}-\int_{K} \boldsymbol{v}(\boldsymbol{x}) \cdot\left(\operatorname{grad} p_{h}^{*}\right)(\boldsymbol{x}) \mathrm{d} \boldsymbol{x}\right) \\
&\left\langle\mathcal{R}_{F}, V\right\rangle= \sum_{K \in \mathcal{T}_{h}^{F}}\left(\int_{K}\left(\boldsymbol{f}_{h}+\nu \Delta u_{h}\right)(\boldsymbol{x}) \cdot \boldsymbol{v}(\boldsymbol{x}) \mathrm{d} \boldsymbol{x}\right. \\
&+\frac{1}{2} \sum_{e \in \mathcal{E}_{K}} \int_{e} \boldsymbol{v}(\boldsymbol{\tau}) \cdot\left[\nu \partial_{n} \boldsymbol{u}_{h}-p_{h} \boldsymbol{n}\right]_{e}(\boldsymbol{\tau}) \mathrm{d} \boldsymbol{\tau} \\
&\left.+\sum_{e \in \mathcal{E}_{K}^{a F}} \int_{e} \boldsymbol{v}(\boldsymbol{\tau}) \cdot\left(\boldsymbol{t}_{a h}-\nu \partial_{h} \boldsymbol{u}_{h}+p_{h} \boldsymbol{n}\right)(\boldsymbol{\tau}) \mathrm{d} \boldsymbol{\tau}+\int_{K}\left(\operatorname{div} \boldsymbol{u}_{h}\right)(\boldsymbol{x}) q(\boldsymbol{x}) \mathrm{d} \boldsymbol{x}\right), \\
&\left\langle\mathcal{R}_{\Gamma}, V\right\rangle=\sum_{e \in \mathcal{E}_{K}^{P, \Gamma}}\left(\int_{e} \boldsymbol{v}(\boldsymbol{\tau}) \cdot\left(\left(p_{h} \boldsymbol{n}\right)_{\mid \Omega_{P}}+\left(\nu \partial_{n} \boldsymbol{u}_{h}-p_{h} \boldsymbol{n}\right)_{\mid \Omega_{F}}\right)(\boldsymbol{\tau}) \mathrm{d} \boldsymbol{\tau}+\int_{\Gamma}(\boldsymbol{v} \cdot \boldsymbol{n})(\boldsymbol{\tau})\left(p_{h \mid \Omega_{P}}^{*}-p_{h \mid \Omega_{P}}\right)(\boldsymbol{\tau}) \mathrm{d} \boldsymbol{\tau}\right) \\
&\langle\mathcal{F}, V\rangle=\int_{\Omega}\left(\boldsymbol{f}-\boldsymbol{f}_{h}\right)(\boldsymbol{x}) \cdot \boldsymbol{v}(\boldsymbol{x}) \mathrm{d} \boldsymbol{x}-\int_{\Gamma_{a P}}(\boldsymbol{v} \cdot \boldsymbol{n})(\boldsymbol{\tau})\left(p_{a}-p_{a h}\right)(\boldsymbol{\tau}) \mathrm{d} \boldsymbol{\tau}+\int_{\Gamma_{a F}} \boldsymbol{v}(\boldsymbol{\tau}) \cdot\left(\mathbf{t}_{a}-\mathbf{t}_{a h}\right)(\boldsymbol{\tau}) \mathrm{d} \boldsymbol{\tau} . \quad(5.16)
\end{aligned}
$$

We can now apply Lemma 5.1 to the function $U-U_{h}^{*}-E_{b}$, which leads to the main result of this section.

Finally, we denote by $\mathcal{E}_{h}^{a F}$ the set of edges or faces of elements of $\mathcal{T}_{h}^{F}$ which are contained in $\bar{\Gamma}_{a F}$. 
Theorem 5.6. Assume that $\Gamma_{F}$ has a positive measure in $\partial \Omega_{F}$, that Assumption 5.2 is satisfied and that the datum $p_{a}$ is continuous on $\bar{\Gamma}_{a P}$. Then the following a posteriori error estimate holds between the solution (u, $\left.p\right)$ of problem (2.6)-(2.7) and the solution $\left(\boldsymbol{u}_{h}, p_{h}\right)$ of problem $(3.9)-(3.10)$

$$
\begin{aligned}
\left\|\boldsymbol{u}-\boldsymbol{u}_{h}\right\|_{X(\Omega)}+\left\|p-p_{h}\right\|_{L^{2}(\Omega)} \leq c\left(\sum_{K \in \mathcal{T}_{h}^{P}}\left(\eta_{K}^{P}\right)^{2}+\sum_{K \in \mathcal{T}_{h}^{F}}\left(\eta_{K}^{F}\right)^{2}+\sum_{e \in \mathcal{E}_{h}^{P, \Gamma}}\left(\eta_{e}^{\Gamma}\right)^{2}\right)^{\frac{1}{2}} \\
+c^{\prime}\left(\left\|k-k_{h}\right\|_{H^{-\frac{1}{2}}\left(\Gamma_{P}\right)}+\left\|\boldsymbol{g}-\boldsymbol{g}_{h}\right\|_{H^{\frac{1}{2}}\left(\Gamma_{F}\right)^{d}}+\left\|\boldsymbol{f}-\boldsymbol{f}_{h}\right\|_{L^{2}\left(\Omega_{P}\right)^{d}}+\left(\sum_{K \in \mathcal{T}_{h}^{F}} h_{K}^{2}\left\|\boldsymbol{f}-\boldsymbol{f}_{h}\right\|_{L^{2}(K)}^{2}\right)^{\frac{1}{2}}\right. \\
\left.+\left\|p_{a}-p_{a h}\right\|_{H_{00}^{\frac{1}{2}}\left(\Gamma_{a P}\right)}+\left(\sum_{e \in \mathcal{E}_{h}^{a F}} h_{e}^{1-2 \gamma_{e}}\left\|\mathbf{t}_{a}-\boldsymbol{t}_{a h}\right\|_{L^{2}(e)^{d}}^{2}\right)^{\frac{1}{2}}\right)
\end{aligned}
$$

Proof. Applying Lemma 5.1 to $U-U_{h}^{*}-E_{b}$ and using equation (5.12) with a further triangle inequality, we are led to bound the quantities

$$
\left\|\boldsymbol{u}_{h}-\boldsymbol{u}_{h}^{*}\right\|_{X(\Omega)}, \quad\left\|p_{h}-p_{h}^{*}\right\|_{L^{2}(\Omega)}, \quad\left\|\boldsymbol{e}_{b}\right\|_{X(\Omega)}
$$

which follows from (5.7), the first part of (5.8) and (5.11), and the term

$$
\sup _{V \in X_{0}(\Omega) \times L^{2}(\Omega)} \frac{\left\langle\mathcal{R}_{P}, V-V_{h}\right\rangle+\left\langle\mathcal{R}_{F}, V-V_{h}\right\rangle+\left\langle\mathcal{R}_{\Gamma}, V-V_{h}\right\rangle+\left\langle\mathcal{F}, V-V_{h}\right\rangle}{\|V\|_{X(\Omega) \times L^{2}(\Omega)}} .
$$

This is obtained by combining Cauchy-Schwarz inequalities with Lemmas 5.4 and 5.5.

To go further, we intend to prove an upper bound for each indicator $\eta_{K}^{P}, \eta_{K}^{F}$ and $\eta_{e}^{\Gamma}$. To do this, we write the residual equation (5.12) in a simpler (and more usual) form: For a smooth enough pair $V=(\boldsymbol{v}, q)$,

$$
\mathcal{A}\left(U-U_{h}, V\right)=\left\langle\mathcal{R}_{P}^{*}, V\right\rangle+\left\langle\mathcal{R}_{F}, V\right\rangle+\left\langle\mathcal{R}_{\Gamma}^{*}, V\right\rangle+\langle\mathcal{F}, V\rangle,
$$

where the new quantities $\mathcal{R}_{P}^{*}$ and $\mathcal{R}_{\Gamma}^{*}$ are defined by

$$
\begin{aligned}
\left\langle\mathcal{R}_{P}^{*}, V\right\rangle=\sum_{K \in \mathcal{T}_{h}^{P}}\left(\int_{K}\left(\boldsymbol{f}_{h}-\alpha \boldsymbol{u}_{h}\right)(\boldsymbol{x}) \cdot \boldsymbol{v}(\boldsymbol{x}) \mathrm{d} \boldsymbol{x}+\frac{1}{2} \sum_{e \in \mathcal{E}_{K}} \int_{e} \boldsymbol{v}(\boldsymbol{\tau}) \cdot \boldsymbol{n}\left[p_{h}\right]_{e}(\boldsymbol{\tau}) \mathrm{d} \boldsymbol{\tau}\right. \\
\left.+\sum_{e \in \mathcal{E}_{K}^{a P}} \int_{e} \boldsymbol{v}(\boldsymbol{\tau}) \cdot \boldsymbol{n}\left(p_{h}-p_{a h}\right)(\boldsymbol{\tau}) \mathrm{d} \boldsymbol{\tau}\right) \\
\left\langle\mathcal{R}_{\Gamma}^{*}, V\right\rangle=\sum_{e \in \mathcal{E}_{K}^{P, \Gamma}} \int_{e} \boldsymbol{v}(\boldsymbol{\tau}) \cdot\left(\left(p_{h} \boldsymbol{n}\right)_{\mid \Omega_{P}}+\left(\nu \partial_{n} \boldsymbol{u}_{h}-p_{h} \boldsymbol{n}\right)_{\mid \Omega_{F}}\right)(\boldsymbol{\tau}) \mathrm{d} \boldsymbol{\tau}
\end{aligned}
$$

However the quantity $\left\langle\mathcal{R}_{P}^{*}, V\right\rangle$ is not defined for all $V$ in $X_{0}(\Omega)$ since it requires a little more regularity.

Proving the upper bounds for the $\eta_{K}^{P}$ is rather standard, it relies on the following choices of the function $V=(\boldsymbol{v}, 0)$ in $(5.18)$ :

- Taking $\boldsymbol{v}$ equal to $\left(\boldsymbol{f}_{h}-\alpha \boldsymbol{u}_{h}\right) \psi_{K}$ on $K$ and to zero elsewhere, where $\psi_{K}$ denotes the bubble function on $K$ equal to the product of the barycentric coordinates associated with the vertices of $K$.

- If the edge $e$ in $\mathcal{E}_{K}$ belongs to another triangle $K^{\prime}$, taking $\boldsymbol{v}$ equal to $\mathcal{R}_{e}\left(\left[p_{h}\right]_{e}\right) \psi_{e}$ on $K \cup K^{\prime}$ and to zero elsewhere. Here $\psi_{e}$ denotes the bubble function on $e$ and $\mathcal{R}_{e}$ is the lifting operator introduced in the proof of Lemma 5.3 and constructed by affine transformation from a fixed lifting operator on a reference triangle.

- Finally, for each $e$ in $\mathcal{E}_{K}^{a P}$, taking $\boldsymbol{v}$ equal to $\mathcal{R}_{e}\left(p_{h}-p_{a h}\right) \psi_{e}$ on $K$ and to zero elsewhere. 


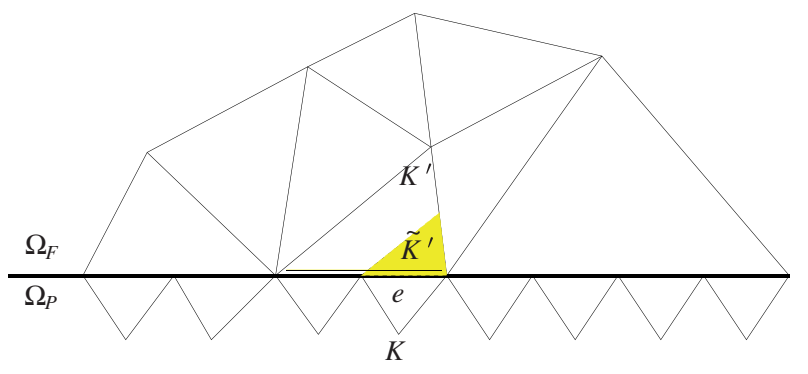

Figure 4. Around an edge $e$ of $\mathcal{E}_{h}^{P, \Gamma}$ (case of dimension $d=2$ ).

Note that the next estimate is not optimal. However this seems due to the choice of the discretization: Indeed, Darcy equations are not dimensionless and the variational formulation that we use in order to couple them with the Stokes problem is not appropriate for handling this difficulty (we refer to [9], Chap. XIII, for a more detailed comparison between the different variational formulations); the same lack of optimality appears in [14] for the Darcy equations only and in [10], Proposition 5.4, for another type of coupling Darcy and Stokes problems.

Proposition 5.7. The following estimate holds for each error indicator $\eta_{K}^{P}$ defined in (5.1), $K \in \mathcal{T}_{h}^{P}$,

$$
\begin{aligned}
\eta_{K}^{P} \leq c\left(\left\|\boldsymbol{u}-\boldsymbol{u}_{h}\right\|_{H\left(\operatorname{div}, \omega_{K}\right)}\right. & +h_{K}^{-1}\left\|p-p_{h}\right\|_{L^{2}\left(\omega_{K}\right)} \\
& \left.+\left\|\boldsymbol{f}-\boldsymbol{f}_{h}\right\|_{L^{2}\left(\omega_{K}\right)^{d}}+h_{K}^{-\frac{1}{2}}\left\|p_{a}-p_{a h}\right\|_{L^{2}\left(K \cap \Gamma_{a P}\right)}\right),
\end{aligned}
$$

where $\omega_{K}$ denotes the union of the elements of $\mathcal{T}_{h}^{P}$ that share at least an edge $(d=2)$ or a face $(d=3)$ with $K$.

Bounding the $\eta_{K}^{F}$ relies again on the residual equation (5.18). The arguments are exactly the same as in [8], Proposition 6, for instance, up to the multiplication by $h_{K}^{-\gamma_{K}}$ and $h_{e}^{-\gamma_{e}}$. So we skip the proof.

Proposition 5.8. The following estimate holds for each error indicator $\eta_{K}^{F}$ defined in $(5.2), K \in \mathcal{T}_{h}^{F}$,

$$
\eta_{K}^{F} \leq c h_{K}^{-\gamma_{K}}\left(\left\|\boldsymbol{u}-\boldsymbol{u}_{h}\right\|_{H^{1}\left(\omega_{K}\right)^{d}}+\left\|p-p_{h}\right\|_{L^{2}\left(\omega_{K}\right)}+h_{K}\left\|\boldsymbol{f}-\boldsymbol{f}_{h}\right\|_{L^{2}\left(\omega_{K}\right)^{d}}+h_{K}^{\frac{1}{2}}\left\|\boldsymbol{t}_{a}-\boldsymbol{t}_{a h}\right\|_{L^{2}\left(K \cap \Gamma_{a F}\right)^{d}}\right)
$$

where $\omega_{K}$ denotes the union of the elements of $\mathcal{T}_{h}^{F}$ that share at least an edge $(d=2)$ or a face $(d=3)$ with $K$.

Proving upper bounds for the $\eta_{e}^{\Gamma}$ requires a further argument.

Proposition 5.9. The following estimate holds for each error indicator $\eta_{e}^{\Gamma}$ defined in $(5.3), e \in \mathcal{E}_{h}^{P, \Gamma}$,

$$
\eta_{e}^{\Gamma} \leq c\left(h_{e}^{-1}\left\|\boldsymbol{u}-\boldsymbol{u}_{h}\right\|_{X\left(\omega_{e}\right)}+h_{e}^{-\frac{1}{2}}\left\|p-p_{h}\right\|_{L^{2}\left(\omega_{e}\right)}+h_{e}^{\frac{1}{2}}\left\|\boldsymbol{f}-\boldsymbol{f}_{h}\right\|_{L^{2}\left(\omega_{e}\right)^{d}}\right),
$$

where $\omega_{e}$ denotes the union of the element of $\mathcal{T}_{h}^{P}$ and of the element of $\mathcal{T}_{h}^{F}$ that share $e$.

Proof. Each $e$ in $\mathcal{E}_{h}^{P, \Gamma}$ is the edge or face of an element $K$ in $\mathcal{T}_{h}^{P}$ and is contained in the edge or face of an element $K^{\prime}$ in $\mathcal{T}_{h}^{F}$. From now on, we denote by $\tilde{K}^{\prime}$ the element contained in $K^{\prime}$ that is constructed from $K^{\prime}$ by homothety and translation and such that $e$ is an edge or a face of $\tilde{K}^{\prime}$ (see Fig. 4). We now prove a bound successively for the two terms in $\eta_{e}^{\Gamma}$. 
1) In (5.18), we take $\boldsymbol{v}$ equal to $\mathcal{R}_{e}\left(\left(p_{h} \boldsymbol{n}\right)_{\mid \Omega_{P}}+\left(\nu \partial_{n} \boldsymbol{u}_{h}-p_{h} \boldsymbol{n}\right)_{\mid \Omega_{F}}\right) \psi_{e}$ on $K \cup \tilde{K}^{\prime}$ and to zero elsewhere, for the already introduced operator $\mathcal{R}_{e}$, and $q$ equal to zero. This yields

$$
\begin{aligned}
\left\|\left(\left(p_{h} \boldsymbol{n}\right)_{\mid \Omega_{P}}+\left(\nu \partial_{n} \boldsymbol{u}_{h}-p_{h} \boldsymbol{n}\right)_{\mid \Omega_{F}}\right) \psi_{e}^{\frac{1}{2}}\right\|_{L^{2}(e)^{d}}^{2} \leq & c\left(\left\|\boldsymbol{u}-\boldsymbol{u}_{h}\right\|_{L^{2}(K)^{d}}\|\boldsymbol{v}\|_{L^{2}(K)^{d}}+\left|\boldsymbol{u}-\boldsymbol{u}_{h}\right|_{H^{1}\left(\tilde{K}^{\prime}\right)^{d}}|\boldsymbol{v}|_{H^{1}\left(\tilde{K}^{\prime}\right)^{d}}\right. \\
& +\left\|p-p_{h}\right\|_{L^{2}(K)^{d}}|\boldsymbol{v}|_{H^{1}(K)^{d}}+\left\|p-p_{h}\right\|_{L^{2}\left(\tilde{K}^{\prime}\right)}|\boldsymbol{v}|_{H^{1}(K)^{d}} \\
& +\left\|\boldsymbol{f}_{h}-\alpha \boldsymbol{u}_{h}\right\|_{\left.L^{2}(K)\right)^{d}}\|\boldsymbol{v}\|_{L^{2}(K)^{d}}+\left\|\boldsymbol{f}_{h}+\nu \Delta \boldsymbol{u}_{h}\right\|_{L^{2}\left(\tilde{K}^{\prime}\right)^{d}}\|\boldsymbol{v}\|_{L^{2}\left(\tilde{K}^{\prime}\right)^{d}} \\
& +\left\|\boldsymbol{f}-\boldsymbol{f}_{h}\right\|_{L^{2}(K)^{d}}\|\boldsymbol{v}\|_{L^{2}(K)^{d}}+\left\|\boldsymbol{f}-\boldsymbol{f}_{h}\right\|_{L^{2}\left(\tilde{K}^{\prime}\right)^{d}}\|\boldsymbol{v}\|_{\left.L^{2}\left(\tilde{K}^{\prime}\right)^{d}\right) .}
\end{aligned}
$$

Note that, in contrast with (5.21), we can obtain an optimal estimate for $\left\|\boldsymbol{f}_{h}-\alpha \boldsymbol{u}_{h}\right\|_{L^{2}(K)^{d}}$. Thus, using several inverse inequalities (see [36], Sect. 3.1, for instance), we derive

$$
\left\|\left(p_{h} \boldsymbol{n}\right)_{\mid \Omega_{P}}+\left(\nu \partial_{n} \boldsymbol{u}_{h}-p_{h} \boldsymbol{n}\right)_{\mid \Omega_{F}}\right\|_{L^{2}(e)^{d}} \leq c h_{e}^{-\frac{1}{2}}\left(\left\|\boldsymbol{u}-\boldsymbol{u}_{h}\right\|_{X\left(\omega_{e}\right)}+\left\|p-p_{h}\right\|_{L^{2}\left(\omega_{e}\right)}+h_{K}\left\|\boldsymbol{f}-\boldsymbol{f}_{h}\right\|_{L^{2}\left(\omega_{e}\right)^{d}}\right) .
$$

2) Let $q$ be a function in $H^{1}\left(K \cup \tilde{K}^{\prime}\right)$ which vanishes on $\partial\left(K \cup \tilde{K}^{\prime}\right)$. By integration by parts, we derive

$$
\int_{e}\left[\boldsymbol{u}_{h} \cdot \boldsymbol{n}\right]_{e}(\boldsymbol{\tau}) q(\boldsymbol{\tau}) \mathrm{d} \boldsymbol{\tau}=\int_{K \cup \tilde{K}^{\prime}}\left(\left(\operatorname{div}\left(\boldsymbol{u}-\boldsymbol{u}_{h}\right)\right)(\boldsymbol{x}) q(\boldsymbol{x})+\left(\boldsymbol{u}-\boldsymbol{u}_{h}\right)(\boldsymbol{x})(\operatorname{grad} q)(\boldsymbol{x}) \mathrm{d} \boldsymbol{x} .\right.
$$

We now take $q$ equal to $\mathcal{R}_{e}\left(\left[\boldsymbol{u}_{h} \cdot \boldsymbol{n}\right]_{e}\right) \psi_{e}$ on $K \cup \tilde{K}^{\prime}$ and to zero elsewhere. Thus, the same inverse inequalities as previously lead to

$$
h_{e}^{-\frac{1}{2}}\left\|\left[\boldsymbol{u}_{h} \cdot \boldsymbol{n}\right]_{e}\right\|_{L^{2}(e)} \leq c\left(\left\|\boldsymbol{u}-\boldsymbol{u}_{h}\right\|_{X\left(\omega_{e}\right)}+h_{e}^{-1}\left\|\boldsymbol{u}-\boldsymbol{u}_{h}\right\|_{L^{2}\left(\omega_{e}\right)^{d}}\right) .
$$

Estimate (5.23) is then derived from (5.24) and (5.25).

There also, estimate (5.23) is not optimal. This seems due to the lack of homogeneity when coupling Darcy and Stokes equations. Indeed, the jump $[\boldsymbol{u} \cdot \boldsymbol{n}]$ on $\Gamma$ is the sum of a function in $H_{00}^{\frac{1}{2}}(\Gamma)^{\prime}$ and of a function in $H^{\frac{1}{2}}(\Gamma)$.

To conclude, we observe that estimate (5.17) is optimal: Up to the terms involving the data, the full error is bounded by a constant times the Hilbertian sum of all indicators. Estimates (5.21), (5.22) and (5.23) are local, i.e., only involve the error in a neighbourhood of $K$ or $e$, and estimate (5.22) is optimal for all elements $K$ that does not intersect $\bar{\Gamma}$. So it can be hoped that the error indicators $\eta_{K}^{P}, \eta_{K}^{F}$ and $\eta_{e}^{\Gamma}$ provide a good tool for mesh adaptivity.

\section{The ADAPtivity STRATEGY AND SOME NUMERICAL EXPERIMENTS}

As standard, the adaptivity strategy that we use combines two steps, an initialization step and an adaptation step. We fix a positive tolerance $\eta^{*}$ and present it in dimension $d=2$ for simplicity.

Initialization STEP. We fix a triangulation $\mathcal{T}_{h}^{P(0)}$ of $\Omega_{P}$ and a triangulation $\mathcal{T}_{h}^{F(0)}$ of $\Omega_{F}$ which satisfy Assumptions 3.1 and 5.2 and such that the sum of the errors on the five data which appear in Theorem 5.6, namely

$$
\begin{aligned}
\left\|k-k_{h}\right\|_{H^{-\frac{1}{2}\left(\Gamma_{P}\right)}}+\left\|\boldsymbol{g}-\boldsymbol{g}_{h}\right\|_{H^{\frac{1}{2}\left(\Gamma_{F}\right)^{d}}}+\left\|\boldsymbol{f}-\boldsymbol{f}_{h}\right\|_{L^{2}\left(\Omega_{P}\right)^{d}}+\left(\sum_{K \in \mathcal{T}_{h}^{F}} h_{K}^{2}\left\|\boldsymbol{f}-\boldsymbol{f}_{h}\right\|_{L^{2}(K)}^{2}\right)^{\frac{1}{2}} \\
+\left\|p_{a}-p_{a h}\right\|_{H_{\mathrm{O}}^{\frac{1}{2}\left(\Gamma_{a P}\right)}}+\left(\sum_{e \in \mathcal{E}_{h}^{a} F} h_{e}^{1-2 \gamma_{e}}\left\|\mathbf{t}_{a}-\boldsymbol{t}_{a h}\right\|_{L^{2}(e)^{d}}^{2}\right)^{\frac{1}{2}},
\end{aligned}
$$




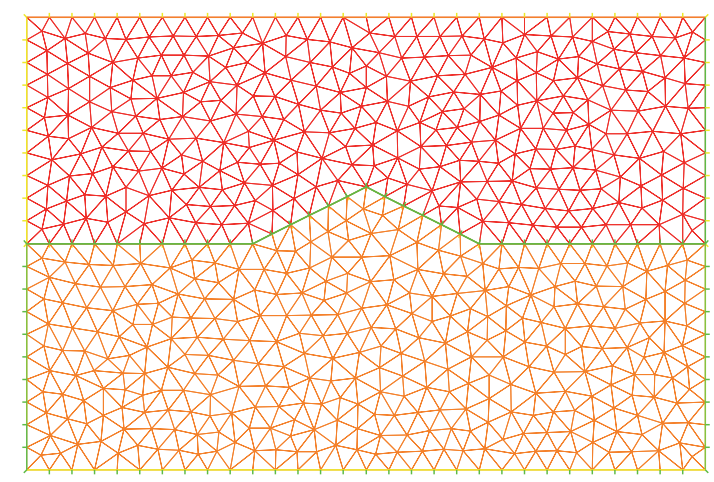

Figure 5. The computation domain and the initial mesh.

is smaller than $\eta^{*}$. This last condition is possible for smooth data thanks to the approximation properties of the finite element spaces involved in the discretization, and we have no applications for non smooth data.

AdAptation step. Assuming that the triangulations $\mathcal{T}_{h}^{P(n)}$ and $\mathcal{T}_{h}^{F(n)}$ are known, we compute the discrete solution of problem (3.9)-(3.10) corresponding to these triangulations, and the error indicators $\eta_{K}^{P}, \eta_{K}^{F}$ and $\eta_{e}^{\Gamma}$ defined in (5.1), (5.2) and (5.3). We denote by $\bar{\eta}^{P}, \bar{\eta}^{F}$ and $\bar{\eta}^{\Gamma}$ the mean values of the $\eta_{K}^{P}$, $\eta_{K}^{F}$ and $\eta_{e}^{\Gamma}$, respectively, times 0.9. Next, we perform adaptivity in three substeps, of course taking into account Assumption 3.1 in all of them:

- All $e$ in $\mathcal{E}_{h}^{P, \Gamma(n)}$ (with obvious notation) such that $\eta_{e}^{\Gamma}$ is larger than $\max \left\{\eta^{*}, \bar{\eta}^{\Gamma}\right\}$ are divided into $N$ equal segments, where $N$ is proportional to the ratio $\eta_{e}^{\Gamma} / \max \left\{\eta^{*}, \bar{\eta}^{\Gamma}\right\}$. This gives rise to a new set $\widetilde{\mathcal{E}}_{h}^{P, \Gamma(n+1)}$.

- The triangulation $\mathcal{T}_{h}^{F(n)}$ is refined and coarsened according to the next criterion: The diameter of a new element contained in $K$ or containing $K$ is proportional to $h_{K}$ times the ratio $\bar{\eta}^{F} / \eta_{K}^{F}$. This gives rise to the new triangulation $\mathcal{T}_{h}^{F(n+1)}$.

- First, the elements of $\widetilde{\mathcal{E}}_{h}^{P, \Gamma(n+1)}$ are divided where needed in order that Assumption 5.2 holds. Second, a new triangulation on $\Omega_{P}$ is constructed such that these new edges are edges of the elements of the new triangulation. Next, adaptivity is performed exactly as in the previous substep, now depending on the ratios $\bar{\eta}^{P} / \eta_{K}^{P}$. This gives rise to the new triangulation $\mathcal{T}_{h}^{P(n+1)}$.

Of course, the adaptation step is iterated either a finite number of times or until the Hilbertian sum of all error indicators, namely

$$
\left(\sum_{K \in \mathcal{T}_{h}^{P}}\left(\eta_{K}^{P}\right)^{2}+\sum_{K \in \mathcal{T}_{h}^{F}}\left(\eta_{K}^{F}\right)^{2}+\sum_{e \in \mathcal{E}_{h}^{P, \Gamma}}\left(\eta_{e}^{\Gamma}\right)^{2}\right)^{\frac{1}{2}}
$$

become smaller than $\eta^{*}$ (this is not always possible).

The numerical experiment that is presented below is performed on the finite element code FreeFem ++ , see [27]. It deals with the two-dimensional domain $\Omega=] 0,3[\times]-2,0\left[\right.$. The top part is the subdomain $\Omega_{F}$ occupied by the river and the bottom part is the subdomain $\Omega_{P}$ occupied by calcalenite rock mixed with sand (so that $\Gamma_{a P}$ is empty). Both $\Omega_{P}$ and $\Omega_{F}$ are nearly equal rectangles, except that a further rock creates an obstacle to the river flow, see Figure 5 . Note also that all the meshes are conforming through the interface $\Gamma$ in order to respect Assumption 5.2; indeed the code that we use cannot enforce this assumption easily. However the computations without this assumption give completely similar results. 


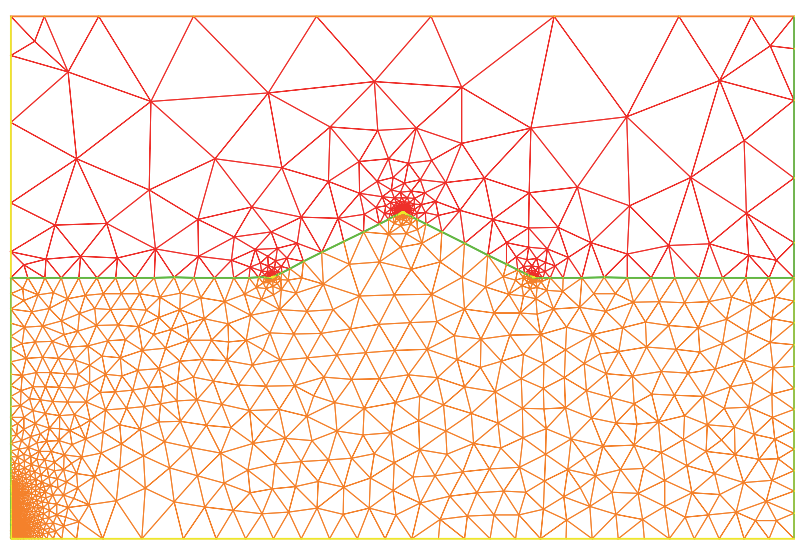

Figure 6. The final adapted mesh.

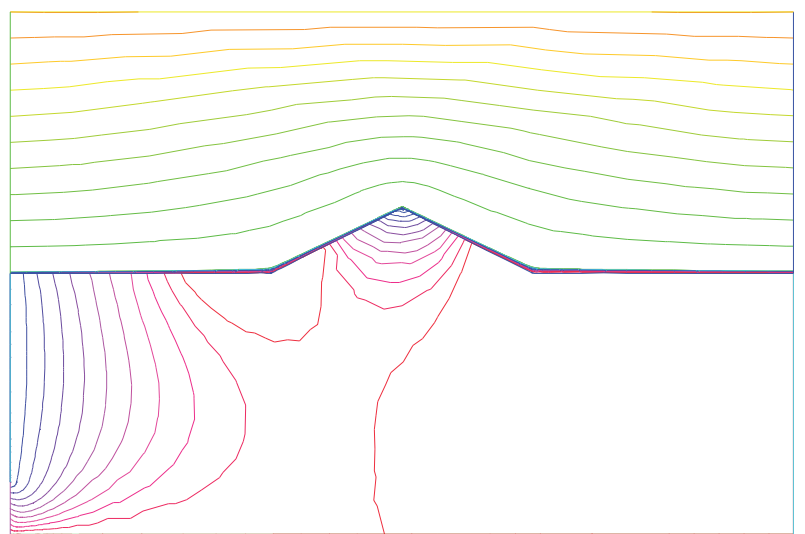

FigURE 7. Isovalues of the streamfunction.

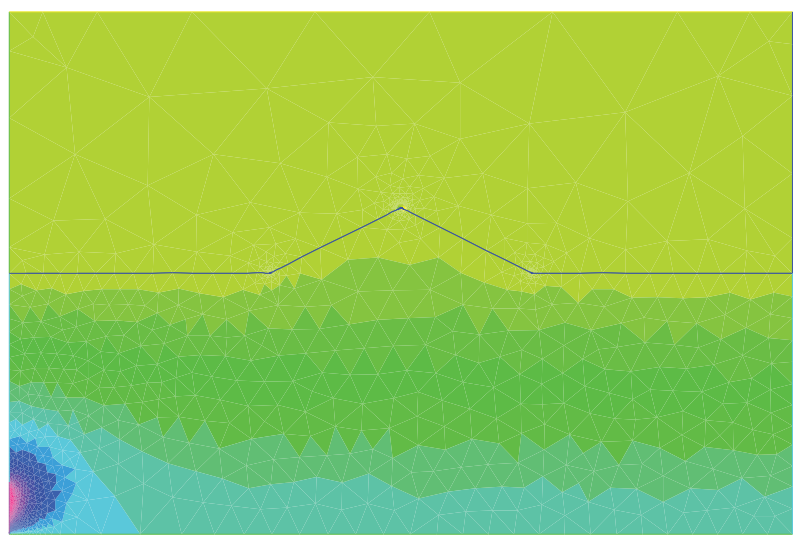

Figure 8. Isovalues of the pressure. 
The parameters $\alpha$ and $\nu$ associated with the permeability of the calcalenite and the viscosity of the water are given by

The data are given by $\boldsymbol{f}=\mathbf{0}$,

$$
\alpha=2000, \quad \nu=0.01
$$

$$
k=\left\{\begin{array}{ll}
0.02 & \text { on }\{0\} \times]-2,-1.8[, \\
0 & \text { elsewhere on } \Gamma_{P},
\end{array} \quad \boldsymbol{g}=\left\{\begin{array}{l}
(1,0) \\
(1.004,0)
\end{array} \quad \text { on }\{3\} \times\right]-1,0\left[, \quad \mathbf{t}_{a}=(0.01,0) \text { on } \Gamma_{a F} .\right.\right.
$$

The non-zero data correspond to an underground spring, the inward and outward flow of the river and the action of the wind, respectively. Note that, in order to preserve the validity of the model, the global flux $\int_{\Gamma_{F}}(\boldsymbol{g} \cdot \boldsymbol{n})(\boldsymbol{\tau}) \mathrm{d} \boldsymbol{\tau}+\int_{\Gamma_{P}} k(\boldsymbol{\tau}) \mathrm{d} \boldsymbol{\tau}$ is zero.

Figure 6 represents the final adapted mesh after 5 iterations of the adaptation step (3412 triangles in $\Omega_{P}$ and 866 triangles in $\left.\Omega_{F}\right)$. Figures 7 and 8 represent the isovalues of the stream function associated with $\boldsymbol{u}_{h}$ and the isovalues of the pressure $p_{h}$, respectively. It must be noted that the flow is much faster in $\Omega_{F}$ than in $\Omega_{P}$. So, to improve the visualization in Figure 7 , we have chosen to use two scales: The difference between two consecutive streamlines is 0.0004 in $\Omega_{P}$ (and there are 20 lines) and 0.1 in $\Omega_{F}$ (and there are only 10 lines). This explains the accumulation of the streamlines in $\Omega_{P}$ near $\Gamma$.

Finally, it appears that the discontinuities of the component $\boldsymbol{u} \cdot \boldsymbol{n}$ through $\Gamma$, issued from the nonconformity of the discretization, are smaller than $10^{-6}$. So using the mortar method to handle this problem seems appropriate.

Acknowledgements. This research work was partially supported by the EU Marie Curie programme and by the Spanish Government grand MTM2006-01275.

\section{REFERENCES}

[1] Y. Achdou and C. Bernardi, Un schéma de volumes ou éléments finis adaptatif pour les équations de Darcy à perméabilité variable. C. R. Acad. Sci. Paris Sér. I 333 (2001) 693-698.

[2] Y. Achdou, C. Bernardi and F. Coquel, A priori and a posteriori analysis of finite volume discretizations of Darcy's equations. Numer. Math. 96 (2003) 17-42.

[3] R.A. Adams, Sobolev Spaces. Academic Press (1975).

[4] S. Antontsev, A.V. Kazhikhov and V.N. Monakhov, Boundary Value Problems in Mechanics of Nonhomogeneous Fluids [English transl.], Studies in Mathematics and its Applications 22. North-Holland (1990).

[5] F. Ben Belgacem, The Mortar finite element method with Lagrangian multiplier. Numer. Math. 84 (1999) $173-197$.

[6] C. Bernardi and G. Raugel, Analysis of some finite elements for the Stokes problem. Math. Comput. 44 (1985) 71-79.

[7] C. Bernardi, Y. Maday and A.T. Patera, A new nonconforming approach to domain decomposition: the mortar element method, in Collège de France Seminar XI, H. Brezis and J.-L. Lions Eds., Pitman (1994) 13-51.

[8] C. Bernardi, B. Métivet and R. Verfürth, Analyse numérique d'indicateurs d'erreur, in Maillage et adaptation, Chap. 8, P.-L. George Ed., Hermès (2001) 251-278.

[9] C. Bernardi, Y. Maday and F. Rapetti, Discrétisations variationnelles de problèmes aux limites elliptiques, Collection Mathématiques et Applications 45. Springer-Verlag (2004).

[10] C. Bernardi, F. Hecht and O. Pironneau, Coupling Darcy and Stokes equations for porous media with cracks. ESAIM: M2AN 39 (2005) 7-35.

[11] C. Bernardi, Y. Maday and F. Rapetti, Basics and some applications of the mortar element method. GAMM - Gesellschaft für Angewandte Mathematik und Mechanik 28 (2005) 97-123.

[12] C. Bernardi, F. Hecht and Z. Mghazli, Mortar finite element discretization for the flow in a non homogeneous porous medium. Comput. Methods Appl. Mech. Engrg. 196 (2007) 1554-1573.

[13] J. Boland and R. Nicolaides, Stability of finite elements under divergence constraints. SIAM J. Numer. Anal. 20 (1983) $722-731$.

[14] D. Braess and R. Verfürth, A posteriori error estimators for the Raviart-Thomas element. SIAM J. Numer. Anal. 33 (1996) 2431-2444.

[15] F. Brezzi and M. Fortin, Mixed and Hybrid Finite Element Methods, Springer Series in Computational Mathematics 15. Springer-Verlag (1991).

[16] E. Burman and P. Hansbo, A unified stabilized method for Stokes' and Darcy's equations. J. Comput. Applied Math. 198 (2007) 35-51. 
[17] D.-G. Calugaru, Modélisation et simulation numérique du transport de radon dans un milieu poreux fissuré ou fracturé. Problème direct et problèmes inverses comme outils d'aide à la prédiction sismique. Ph.D. thesis, Université de FrancheComté, Besançon, France (2002).

[18] C. Carstensen and J. Hu, A unifying theory of a posteriori error control for nonconforming finite element methods. Numer. Math. 107 (2007) 473-502.

[19] M. Dauge, Neumann and mixed problems on curvilinear polyhedra. Integr. Equat. Oper. Th. 15 (1992) $227-261$.

[20] G. de Marsily, Quantitative Hydrology. Groundwater Hydrology for Engineers. Academic Press, New York (1986).

[21] M. Discacciati, E. Miglio and A. Quarteroni, Mathematical and numerical models for coupling surface and groundwater flows. Appl. Numer. Math. 43 (2002) 57-74.

[22] M. Fortin, Old and new elements for incompressible flows. Internat. J. Numer. Methods Fluids 1 (1981) 347-364.

[23] J. Galvis and M. Sarkis, Non-matching mortar discretization analysis for the coupling Stokes-Darcy equations. (Submitted).

[24] V. Girault and P.-A. Raviart, Finite Element Methods for Navier-Stokes Equations, Theory and Algorithms, Springer Series in Computational Mathematics 5. Springer-Verlag (1986).

[25] V. Girault, R. Glowinski and H. López, A domain decomposition and mixed method for a linear parabolic boundary value problem. IMA J. Numer. Anal. 24 (2004) 491-520.

[26] P. Grisvard, Elliptic Problems in Nonsmooth Domains. Pitman (1985).

[27] F. Hecht and O. Pironneau, FreeFem++, see www.freefem.org.

[28] W.J. Layton, F. Schieweck and I. Yotov, Coupling fluid flow with porous media flow. SIAM J. Numer. Anal. 40 (2002) 2195-2218.

[29] R. Lewandowski, Analyse mathématique et océanographie, Collection Recherches en Mathématiques Appliquées. Masson (1997).

[30] J.-L. Lions and E. Magenes, Problèmes aux limites non homogènes et applications, Vol. 1. Dunod (1968).

[31] J.-C. Nédélec, Mixed finite elements in $\mathbb{R}^{3}$. Numer. Math. 35 (1980) 315-341.

[32] M. Orlt and A.-M. Sändig, Regularity of viscous Navier-Stokes flows in nonsmooth domains, in Proc. Conf. Boundary Value Problems and Integral Equations in Nonsmooth Domains, M. Costabel, M. Dauge and S. Nicaise Eds., Lect. Notes Pure Appl. Math. 167, Dekker (1995) 185-201.

[33] K.R. Rajagopal, On a hierarchy of approximate models for flows of incompressible fluids through porous solids. Math. Models Methods Appl. Sci. 17 (2007) 215-252.

[34] P.-A. Raviart and J.-M. Thomas, A mixed finite element method for second order elliptic problems, in Mathematical Aspects of Finite Element Methods, Lect. Notes Math. 606, Springer (1977) 292-315.

[35] J.M. Urquiza, D. N'Dri, A. Garon and M.C. Delfour, Coupling Stokes and Darcy equations. Applied Numer. Math. (2007) (in press).

[36] R. Verfürth, A Review of A Posteriori Error Estimation and Adaptive Mesh-Refinement Techniques. Wiley \& Teubner (1996). 